\title{
GEOMETRY AND A PRIORI ESTIMATES FOR FREE BOUNDARY PROBLEMS OF THE EULER'S EQUATION
}

\author{
JALAL SHATAH ${ }^{\dagger}$ AND CHONGCHUN ZENG*
}

\begin{abstract}
In this paper we derive estimates to the free boundary problem for the Euler equation with surface tension, and without surface tension provided the Rayleigh-Taylor sign condition holds. We prove that as the surface tension tends to zero, when the Rayleigh-Taylor condition is satisfied, solutions converge to the Euler flow with zero surface tension.
\end{abstract}

\section{INTRODUCTION}

In this paper we study free boundary problems of the Euler's equation in vacuum:

$$
\begin{cases}v_{t}+\nabla_{v} v=-\nabla p, & x \in \Omega_{t} \subset \mathbb{R}^{n} \\ \nabla \cdot v=0, & x \in \Omega_{t} .\end{cases}
$$

where for every $t \in \mathbb{R}, v(t, \cdot)$ is the velocity field of an incompressible inviscid fluid in a moving domain (bounded and connected) $\Omega_{t} \subset \mathbb{R}^{n}, n \geq 2$, and $p(t, \cdot)$ is the pressure. The boundary of the domain $\Omega_{t}$ moves with the fluid velocity and the pressure at the boundary is given by the surface tension, that is

$$
\left\{\begin{array}{l}
\mathbf{D}_{t}=\partial_{t}+v \cdot \nabla \quad \text { is tangent to } \quad \bigcup_{t} \Omega_{t} \subset \mathbb{R}^{n+1} \\
p(t, x)=\epsilon^{2} \kappa(t, x), \quad x \in \partial \Omega_{t}, \quad 0 \leq \epsilon \leq 1
\end{array}\right.
$$

where $\kappa(t, x)$ is the mean curvature of the boundary $\partial \Omega_{t}$ at $x \in \partial \Omega_{t}$, and $\mathbf{D}_{t}$ is the material derivative. This is equivalent to saying the velocity of $\partial \Omega_{t}$ is given by $v \cdot N$ where $N$ is the unit normal to $\partial \Omega_{t}$. The case $\epsilon=0$ corresponds to the zero surface tension problem.

In the presence of surface tension we will derive energy estimates that bound the Sobolev norms of the velocity and the boundary. In addition we will show that if the Rayleigh-Taylor sign condition is verified, then some of these bounds are independent of $\epsilon$. In this case we conclude that as $\epsilon \rightarrow 0$ solutions of the problem with surface tension converge to solutions of the zero surface tension problem. We do not include the effects of gravity in (E) as it will only contribute lower order terms to our estimates.

The free boundary value problem for (E) has been studied intensively by many authors. In the absence of surface tension the earliest mathematical results on the well posedness of the water waves problem were given by V. I. Nalimov [NA74] where he considered the irrotational problem in 2 dimensions with small data in some Sobolev space (see also H. Yoshihara [YO82]). The first break through in solving the well posedness for the irrotational problem, no surface tension, for general data came in the work of S. J. Wu [WU97, WU99] who solved the problem in all dimensions. For the general problem with no surface tension D. Christodoulou and H. Lindblad [CL00] were the first to obtain energy estimates based on the geometry of the moving domain, assuming the Rayleigh-Taylor sign condition for rotational flows. H. Lindblad [LI05] proved existence of solutions for the general problem. In the absence of this condition D. Ebin [EB87] proved that the problem

$\dagger$ The first author is funded in part by NSF DMS 0203485.

* The second author is funded in part by NSF DMS 0627842 and the Sloan Fellowship. 
is ill-posed. There is also the work of K. Beyer and M. Günther [BG98, BG00] on well posedness which we will comment more on at the end of the introduction.

For the problem with surface tension H. Yoshihara [YO83], T. Iguchi [IG01] and D. Ambrose [AM03] solved the well posedness irrotational problem in 2 dimensions under varying assumptions on the initial data. B. Schweizer [SC05] proved existence for the general 3 dimensional problem. Recently D. Ambrose and N. Masmoudi [AM05a] proved that as $\epsilon \rightarrow 0$ solutions of the 2 dimensional irrotational problem converges to solutions of the zero surface problem by writing the equation in terms of the arc length of the fluid boundary.

There are many other works on this problem we mention the work of D. Lannes [LA05], T. J. Beal, T. Hou, and J. Lowengrub [BHL93], W. Craig [CR85], G. Shnider and E. C. Wayne [SW02], M. Ogawa and A. Tani [OT02].

During the writing of this manuscript we were informed and received reprints of several related work by D. Coutand and S. Shkoller [SC05], D. Ambrose and N. Mamoudi [AM06], and P. Zhang and Z. Zhang [ZZ06]. In D. Coutand and S. Shkoller work they proved local well posedness of the general problem using Lagrangian coordinates.

Our results differs from those mentioned above in that we can obtain the $\epsilon \rightarrow 0$ limit for the general problem. Our approach to the problem is based on the well known fact that the free boundary problem (E, BC) has a Lagrangian formulation given by

$$
I(u)=\iint_{\Omega_{0}} \frac{\left|u_{t}\right|^{2}}{2} d y d t-\epsilon^{2} \int S(u) d t
$$

where $u(t, \cdot) \in \Gamma=\left\{\Phi: \Omega_{0} \rightarrow \mathbb{R}^{n}\right.$, volume preserving homeomorphisms $\}$, and $S(u)$ is the surface area of $u\left(\partial \Omega_{t}\right)$. Critical points of $I$ satisfy

$$
\partial_{t} u_{t}+q+\epsilon^{2} S^{\prime}(u)=0
$$

where $q$ is the Lagrange multiplier due to the constraint $u \in \Gamma$. Writing $v=u_{t} \circ u^{-1}$ and changing to Eulerian coordinates we obtain

$$
\partial_{t} u_{t} \rightarrow \mathbf{D}_{t} v, \quad q \rightarrow \nabla p_{v, v}=-\nabla \Delta^{-1} \operatorname{tr}(D v D v), \quad S^{\prime}(u) \rightarrow J \triangleq \nabla \kappa_{\mathcal{H}}
$$

where $\Delta^{-1}$ is the inverse Laplacian with zero Dirichlet data, $\kappa$ the mean curvature, and $\kappa_{\mathcal{H}}$ is the harmonic extension of $\kappa$ into $\Omega_{t}$. Thus (E-L) is the Euler equation with the pressure $p$ given by

$$
p=p_{v, v}+\epsilon^{2} \kappa_{\mathcal{H}} .
$$

It is important to note that this derivation splits the pressure into two terms, the first $p_{v, v}$ is the Lagrange multiplier, and the second $\kappa_{\mathcal{H}}$ is due to surface tension. Thus these two terms will be treated differently in the energy estimates.

Using this variational derivation, one can interpret the Lagrange multiplier as the second fundamental form of the manifold $\Gamma \subset L^{2}\left(\Omega_{0}, \mathbb{R}^{n}\right)$ and rewrite $(\mathrm{E}-\mathrm{L})$ as ${ }^{\dagger}$

$$
\overline{\mathscr{D}}_{t} u_{t}+\epsilon^{2} S^{\prime}(u)=0 \text {. }
$$

where $\overline{\mathscr{D}}$ is the Riemannian connection induced on $\Gamma$ by the embedding in $L^{2}$. The above form of the equation makes it relatively easy to identify the correct linearized problem

$$
\overline{\mathscr{D}}_{t}^{2} \bar{w}+\overline{\mathscr{R}}\left(u_{t}, \bar{w}\right) u_{t}+\epsilon^{2} \overline{\mathscr{D}}^{2} S(u)(\bar{w})=0, \quad \bar{w}(t, \cdot) \in T_{u(t, \cdot)} \Gamma,
$$

where $\overline{\mathscr{R}}$ is the curvature tensor of the infinite dimensional manifold $\Gamma \subset L^{2}$. Keeping the highest order terms in the above equation we obtain

$$
\overline{\mathscr{D}}_{t}^{2} \bar{w}+\overline{\mathscr{R}}_{0}(v) \bar{w}+\epsilon^{2} \overline{\mathscr{A}} \bar{w}=\text { lower order terms, }
$$

\footnotetext{
${ }^{\dagger}$ Symbols in the Lagrangian description have a bar, e.g. $\overline{\mathscr{D}}$, while their Eulerian counterparts do not, $\mathscr{D}$.
} 
where $\overline{\mathscr{R}}_{0}(v)$ is a first order differential operator and $\overline{\mathscr{A}}$ is a third order differential operator. In Eulerian coordinates these terms are given by

$$
\begin{aligned}
& \mathscr{R}_{0}(v)(w, w)=\int_{\partial u\left(\Omega_{0}\right)}-\nabla_{N} p_{v, v}|w \cdot N|^{2} d S, \\
& \mathscr{A}(u)(w, w)=\int_{\partial u\left(\Omega_{0}\right)}\left|\nabla^{\top} w \cdot N\right|^{2} d S
\end{aligned}
$$

where $N$ is the unit normal and $\nabla^{\top}$ is the tangential gradient on the boundary of $u\left(\Omega_{0}\right)$. Here once again we are led in a natural way to distinguish the two problems in the following manner.

1) For $\epsilon>0$ two time derivatives are associated with $\overline{\mathscr{A}}$, which is a positive semi-definite operator similar to three spatial differentiation, thus roughly speaking, $\partial_{t} \sim\left(\partial_{x}\right)^{\frac{3}{2}}$. Therefore one may be led to believe that the regularity of the Lagrangian coordinates given by $\partial_{t} u=v$ is $\frac{3}{2}$ order better than $v$, which reflects the regularizing effect of the surface tension. However this is not true for the Lagrangian coordinates since $\mathscr{A}$ is degenerate, and the regularity improvement of the $\partial \Omega_{t}$ is geometric and is not reflected in the Lagrangian coordinates system . See Section 5 for examples. Thus Eularian coordinates are more suitable to use than Lagrangian coordinates for our estimates. 2) For $\epsilon=0$ the leading term involves $\mathscr{R}_{0}(v)$ and thus the Rayleigh-Taylor instability may occur unless we impose the condition

$$
-\nabla_{N} p_{v, v}(t, x)>a>0 \quad x \in \partial \Omega_{t} .
$$

In this case two time derivatives are associated with $\overline{\mathscr{R}}$ which is a positive semi-definite operator similar to one spatial differentiation. Thus, $\partial_{t} \sim\left(\partial_{x}\right)^{\frac{1}{2}}$ and comments similar to above hold on the regularity of $\partial \Omega_{t}$.

3) For $\epsilon>0$ one can directly obtain nonlinear estimates that depend on $\epsilon$ by multiplying (E-L) by $\left(\mathscr{D}^{2} S\right)^{k} S^{\prime}$.

4)The control that any power $\mathscr{R}_{0}(v)$, with (RT) condition, can give over vector fields is limited by the smoothness of the boundary $\partial \Omega_{t}$. This fact makes the velocity field $v$ inappropriate vector field to estimate because it is smoother than what these operators allow.

5) Since $\mathscr{A}$ and $\mathscr{R}_{0}(v)$ are degenerate for fields which are tangential to the boundary $\partial \Omega_{t}$ one needs to add the vorticity $\omega$ which controls the rotational part of the velocity which is tangential to the boundary.

These facts imply that a natural energy to control is

$$
\mathcal{E}=\int_{\Omega} \frac{1}{2}\left|\mathscr{A}^{k-1} \mathscr{D}_{t} J\right|^{2}+\frac{\epsilon^{2}}{2}\left|\mathscr{A}^{k-\frac{1}{2}} J\right|^{2} d x+\frac{1}{2} \mathscr{R}_{0}(v)\left(\mathscr{A}^{k-1} J, \mathscr{A}^{k-1} J\right)+|\omega|_{H^{3 k-1}(\Omega)}^{2}
$$

where $J=\nabla \kappa_{\mathcal{H}}$ is less smooth than $v$ and satisfies

$$
\overline{\mathscr{D}}_{t}^{2} \bar{J}+\overline{\mathscr{R}}_{0}(v) \bar{J}+\epsilon^{2} \overline{\mathscr{A}} \bar{J}=\text { lower order terms, }
$$

In addition to the geometry of $\Gamma$ the geometry of $\partial \Omega_{t}$ plays a crucial role in the estimates. The appearance of $\kappa$ in the surface tension and $\nabla \kappa_{\mathcal{H}}$ in the energy make the study of the geometry of the boundary as well as the study of the harmonic extension and Dirichlet-Neumann operators on $\partial \Omega_{t}$ central to the estimate. In using Lagrangian coordinates these operators may be hidden but can not be avoided.

Based on these estimates one can construct an existence proof using the following iteration method. Since the acceleration of the boundary is given by the surface tension plus lower order terms, in the first step of the iteration we evolve the boundary using this evolution. In the second step of the iteration we establish the evolution of the velocity in the interior. This will appear in a forthcoming paper.

Finally after this work was completed A. Mielke pointed out to the second author that a similar geometric approach had been used by K. Beyer and M. Günther to study the irrotational problem 
by reducing it to the boundary[BG98, BG00]. Indeed they proved local well posedness for star shaped domains with surface tension and studied the linearized flow for any irrotational flow. In fact they derived the principle part of the curvature of $\Gamma$ for irrotational problem which of course coincides with our $\mathscr{R}_{0}(v)$ acting on gradient vector fields.

This paper is organized as follows. In section 2 of the paper we give the intuition behind the energy estimates by computing the geometry of $\Gamma$. In section 3 we present the geometric computation of the moving boundary. In section 4 we present our energy estimates. In sections 5 and 6 we present some examples and basic analytic and geometric calculations.

Notations. All notations will be defined as they are introduced. In addition a list of symbols will be given at the end of the paper for a quick reference. Here we'll present some standard notations and conventions used throughout the paper.

All constants will be denoted by $C$ which is a generic bound depending only on the quantities specified in the context. We follow the Einstein convention where we sum upon repeated indices.

For a domain $\Omega_{t}$ and $x \in \partial \Omega_{t}$ we denote by $N(t, x)$ the outward unit normal, $\Pi$ the second fundamental form where $\Pi(w)=\nabla_{w} N \in T_{x} \partial \Omega_{t}$ for $w \in T_{x} \partial \Omega_{t}$, and $\kappa$ the mean curvature given by the trace of $\Pi$, i.e., $\kappa=\operatorname{tr} \Pi$. The regularity of the domains $\Omega_{t}$ is characterized by the local regularity of $\partial \Omega$ as graphs. In general, an $m$-dimensional manifold $\mathcal{M} \subset \mathbb{R}^{n}$ is said to be of class $C^{k}$ or $H^{s}, s>\frac{n}{2}$, if, locally in linear frames, $\mathcal{M}$ can be represented by graphs of $C^{k}$ or $H^{s}$ mappings, respectively. For $\partial \Omega$, throughout this paper we will only use these local graph coordinates in orthonormal frames.

\section{The GeOmetry Behind the Energy}

In this section, we heuristically outline our geometric point of view on the free boundary problems of the Euler's equation and the intuition leading to the energy estimates in the following two sections. Though the discussion in this section are mostly in Lagrangian coordinates, the estimates are actually done in Eulerian coordinates in the next two sections.

2.1. Lagrangian formulation of the problem. One of the fundamental properties of the inviscid fluid motion is the law of energy conservation. Multiplying the Euler's equation (E) by $v$, integrating on $\Omega_{t}$, and using (BC), we obtain the conserved energy $E_{0}$ :

$$
E_{0}=E_{0}\left(\Omega_{t}, v(t, \cdot)\right)=\int_{\Omega_{t}} \frac{|v|^{2}}{2} d x+\epsilon^{2} \int_{\partial \Omega_{t}} d S \triangleq \int_{\Omega_{t}} \frac{|v|^{2}}{2} d x+\epsilon^{2} S\left(\partial \Omega_{t}\right) .
$$

The main difficulty of these problems is handling the free boundary. A traditional way to avoid this difficulty is to consider the Lagrangian coordinates. Let $u(t, y), y \in \Omega_{0}$, be the Lagrangian coordinate map solving

$$
\frac{d x}{d t}=v(t, x), \quad x(0)=y,
$$

then we have $v=u_{t} \circ u^{-1}$ and for any vector field $w(t, x), x \in \Omega_{t}$, it is clear that

$$
\mathbf{D}_{t} w \triangleq \partial_{t} w+\nabla_{v} w=(w \circ u)_{t} \circ u^{-1}
$$

Therefore, the Euler's equation can be rewritten as

$$
u_{t t}=-(\nabla p) \circ u, \quad u(0)=i d_{\Omega_{t}}, \quad-\Delta p=\operatorname{tr}\left((D v)^{2}\right),\left.\quad p\right|_{\Omega_{t}}=\kappa,
$$

where $\kappa$ is the mean curvature of $\partial \Omega_{t}$.

Since $v(t, \cdot)$ is divergence free, then $u(t, \cdot)$ is volume preserving. Let

$$
\Gamma \triangleq\left\{\Phi: \Omega_{0} \rightarrow \mathbb{R}^{n} \mid \Phi \text { is a volume preserving homeomorphism }\right\} .
$$

As a manifold, the tangent space of $\Gamma$ is given by divergence free vector fields:

$$
T_{\Phi} \Gamma=\left\{\bar{w}: \Omega_{0} \rightarrow \mathbb{R}^{n} \mid \nabla \cdot w=0, \text { where } w=\left(\bar{w} \circ \Phi^{-1}\right)\right\} .
$$


For the remainder of this section we follow the following convention: for any vector field $X$ : $\Phi\left(\Omega_{0}\right) \rightarrow \mathbb{R}^{n}$ its description in Lagrangian coordinates is given by $\bar{X}=X \circ \Phi$. With slight abuse of notation, we also let $S(\Phi)=\int_{\partial \Phi\left(\Omega_{0}\right)} d S$, i.e. the surface area of $\Phi\left(\Omega_{0}\right)$. Thus, the energy $E_{0}$ takes the following form in the Lagrangian coordinates:

$$
E_{0}=E_{0}\left(u, u_{t}\right)=\frac{1}{2} \int_{\Omega_{0}}\left|u_{t}\right|^{2} d y+\epsilon^{2} S(u), \quad\left(u, u_{t}\right) \in T \Gamma
$$

where the volume preserving property of $u$ is used. This conservation of energy suggests: 1) T $\Gamma$ be endowed with the $L^{2}$ metric; and 2) the free boundary problem of the Euler's equation has a Lagrangian action

$$
I(u)=\iint_{\Omega_{0}} \frac{\left|u_{t}\right|^{2}}{2} d x d t-\epsilon^{2} \int S(u) d t, \quad u(t, \cdot) \in \Gamma .
$$

Let $\overline{\mathscr{D}}$ denote the covariant derivative associated with the metric on $\Gamma$, then a critical path $u(t, \cdot)$ of $I$ satisfies

$$
\overline{\mathscr{D}}_{t} u_{t}+\epsilon^{2} S^{\prime}(u)=0 .
$$

In order to verify that the Lagrangian coordinate map $u(t, \cdot)$ of a solution of $(\mathrm{E})$ and $(\mathrm{BC})$ is indeed a critical path of $I$, it is convenient to calculate $\overline{\mathscr{D}}$ and $S^{\prime}$ by viewing $\Gamma$ as a submanifold of the Hilbert space $L^{2}\left(\Omega_{0}, \mathbb{R}^{n}\right)$.

Computing $\left(T_{\Phi} \Gamma\right)^{\perp}$. For any vector field $X: \Phi(\Omega) \rightarrow \mathbb{R}^{n}$ we form the Hodge decomposition

$$
X=w-\nabla \psi, \quad \psi=-\Delta^{-1} \nabla \cdot X, \quad \nabla \cdot w=0,
$$

where $\Delta^{-1}$ is the inverse Laplacian on $\Omega_{t}$ with zero Dirichlet data. Therefore if $\Phi \in \Gamma$, then $\bar{w}=w \circ \Phi \in T_{\Phi} \Gamma$. This implies that normal space of $T_{\Phi} \Gamma$ at $\Phi$ is

$$
\left(T_{\Phi} \Gamma\right)^{\perp}=\left\{-(\nabla \psi) \circ \Phi|\psi|_{\partial\left(\Phi\left(\Omega_{0}\right)\right)} \equiv 0\right\} .
$$

since the Hodge decomposition is orthogonal in $L^{2}$ and $\Phi$ is volume preserving.

Computing $\mathscr{D}_{t}$. Given a path $u(t, \cdot) \in \Gamma$ and $\bar{v}=u_{t}$. Suppose $\bar{w}(t, \cdot) \in T_{u(t)} \Gamma$, then the covariant derivative $\overline{\mathscr{D}}_{t} \bar{w}$ and the second fundamental form $I I_{u(t)}(\bar{w}, \bar{v})$ satisfy

$$
\bar{w}_{t}=\overline{\mathscr{D}}_{t} \bar{w}+I I_{u(t)}(\bar{w}, \bar{v}), \quad \overline{\mathscr{D}}_{t} \bar{w} \in T_{u(t)} \Gamma, \quad I I_{u(t)}(\bar{w}, \bar{v}) \in\left(T_{u(t)} \Gamma\right)^{\perp} .
$$

Let $v=u_{t} \circ u^{-1}=\bar{v} \circ u^{-1}$ and $w=\bar{w} \circ u^{-1}$ which are in the Eulerian coordinates. Then from the Hodge decomposition we have

$$
\overline{\mathscr{D}}_{t} \bar{w}=\bar{w}_{t}-I I(\bar{w}, \bar{v}), \quad I I(\bar{w}, \bar{v})=-\left(\nabla p_{w, v}\right) \circ u, \quad p_{w, v}=-\Delta^{-1} \operatorname{tr}(D w D v) .
$$

As we do the estimates in the Eulerian coordinates, sometimes it is more convenient to use

$$
\mathscr{D}_{t} w=\left(\overline{\mathscr{D}}_{t} \bar{w}\right) \circ u^{-1}=\mathbf{D}_{t} w+\nabla p_{w, v} \text {. }
$$

Computing $S^{\prime}(u)$. By the variation of surface area formula and for any $\bar{w} \in T_{u} \Gamma$ we have

$$
<S^{\prime}(u), \bar{w}>_{L^{2}\left(\Omega_{0}\right)}=\int_{\partial \Omega_{t}} \kappa(w)^{\perp} d S=\int_{\Omega_{t}} \nabla \kappa_{\mathcal{H}} \cdot w d x
$$

where $\kappa$ is the mean curvature, and $\kappa_{\mathcal{H}}$ is its harmonic extension. Since $\left(\nabla \kappa_{\mathcal{H}}\right) \circ u \in T_{u} \Gamma$, we obtain

$$
S^{\prime}(u)=\left(\nabla \kappa_{\mathcal{H}}\right) \circ u \triangleq J \circ u .
$$

This vector field $J$, divergence free on $\Omega_{t}$, is very important for it connects the free boundary Euler's flow with the geometry of $\partial \Omega_{t}$ and even of $\Gamma$ as we will see later in section 3.2.

Combining (2.10) and (2.7) with $\bar{w}=u_{t}$, we obtain that the equation (2.6) for critical paths of $I$ becomes

$$
u_{t t}=\mathbf{D}_{t} v \circ u=\left(-\nabla p_{v, v}-\epsilon^{2} J\right) \circ u, \quad v=u_{t} \circ u^{-1},
$$


which is equivalent to (2.4). Therefore, the free boundary problem (E) and (BC) is a lagrangian system on $\Gamma$ given by (2.6). If $\epsilon=0$ equation (2.6) becomes the geodesic equation on $\Gamma$, which is a well-known fact.

2.2. Linearization. In order to analyze the free boundary problems of the Euler's equation, it is natural to start with the linearization. The Lagrangian formulation provides a convenient frame work for this purpose. From (2.6), the linearized equation is

$$
\overline{\mathscr{D}}_{t}^{2} \bar{w}+\overline{\mathscr{R}}\left(u_{t}, \bar{w}\right) u_{t}+\epsilon^{2} \overline{\mathscr{D}}^{2} S(u)(\bar{w})=0, \quad \bar{w}(t, \cdot) \in T_{u(t, \cdot)} \Gamma,
$$

where $\overline{\mathscr{R}}$ is the curvature tensor of the infinite dimensional manifold $\Gamma$. Below we calculate $\overline{\mathscr{D}}^{2} S(u)$, which is viewed as a linear operator on $T_{u} \Gamma$, and $\overline{\mathscr{R}}$.

Computing $\overline{\mathscr{D}}^{2} S(u)$. Let $g(s, \cdot)$ be a geodesic on $\Gamma, g(0)=u$. Let $\bar{w}=g_{s}$ and $\Omega_{s}=g(s, \cdot)\left(\Omega_{0}\right)$. From (2.8) we have $\overline{\mathscr{D}}_{s} \bar{w}=\left(\mathbf{D}_{s} w+\nabla p_{w, w}\right) \circ g=0$. Differentiating (2.9),

$$
\overline{\mathscr{D}}^{2} S(u)(\bar{w}, \bar{w})=\frac{d}{d s} \int_{\partial \Omega_{s}} \kappa w \cdot N d S .
$$

and substitute the expressions for $D_{s} N, D_{s} S$, and $D_{s} \kappa$ from (3.1), (3.2), and (3.7) we obtain

$$
\begin{aligned}
\overline{\mathscr{D}}^{2} S(u)(\bar{w}, \bar{w})= & \int_{\partial \Omega_{s}} \kappa w^{\perp}\left(\kappa w^{\perp}+\mathcal{D} \cdot w^{\top}\right)+\kappa \mathbf{D}_{s} w \cdot N+\kappa w \cdot \mathbf{D}_{s} N+w^{\perp} \mathbf{D}_{s} \kappa d S \\
= & \int_{\partial \Omega_{s}} \kappa w^{\perp}\left(\kappa w^{\perp}+\mathcal{D} \cdot w^{\top}\right)-\kappa \nabla_{N} p_{w, w}-\kappa \nabla_{w^{\top}} w \cdot N \\
& +w^{\perp}\left(-\Delta_{\partial \Omega_{s}} w^{\perp}-w^{\perp}|\Pi|^{2}+(\mathcal{D} \cdot \Pi)\left(w^{\top}\right)\right) d S,
\end{aligned}
$$

where $\Pi$ is the second fundamental form of $\partial \Omega_{s}$.

Needless to say that this is a very complicated expression for $\overline{\mathscr{D}}^{2} S(u)(\bar{w}, \bar{w})$. We will show that $\overline{\mathscr{D}}^{2} S(u)$ is a differential operator and will single out its leading order part. Let us assume that $\Omega_{s}$ is a sufficiently smooth domain, then from the trace theorem,

$$
\left.\left|\bar{D}^{2} S(u)(\bar{w}, \bar{w})-\int_{\partial \Omega_{s}}\right| \nabla^{\top} w^{\perp}\right|^{2}+\left.\kappa \nabla_{N} p_{w, w} d S|\leq C| w\right|_{H^{1}\left(\Omega_{s}\right)} ^{2} .
$$

The third term on the left side is estimated by applying the Divergence Theorem twice,

$$
\int_{\partial \Omega_{s}} \kappa \nabla_{N} p_{w, w} d S=\int_{\Omega_{s}} \nabla \kappa_{\mathcal{H}} \cdot \nabla p_{w, w}-\kappa_{\mathcal{H}} \operatorname{tr}(D w)^{2} d x=\int_{\partial \Omega_{s}} \nabla_{w} w \cdot \nabla \kappa_{\mathcal{H}} d x-\int_{\partial \Omega_{s}} \kappa \nabla_{w} w \cdot N d S
$$

Therefore, we obtain

$$
\left.\left.\left|\overline{\mathscr{D}}^{2} S(u)(\bar{w}, \bar{w})-\int_{\partial \Omega_{s}}\right| \nabla^{\top} w^{\perp}\right|^{2} d S|\leq C| w\right|_{L^{2}\left(\partial \Omega_{s}\right)}|w|_{H^{1}\left(\partial \Omega_{s}\right)} \leq C|w|_{H^{1}\left(\Omega_{s}\right)}^{2} .
$$

Much as in the derivation of (2.9), for a general $u \in \Gamma$ we derive an self-adjoint operator $\overline{\mathscr{A}}(u)$ on $T_{u} \Gamma$

which satisfies

$$
\overline{\mathscr{A}}(u)(\bar{w})=\left(\nabla \mathcal{H}\left(-\Delta_{\partial u\left(\Omega_{0}\right)}\left(\left.w\right|_{\partial u\left(\Omega_{0}\right)}\right)^{\perp}\right)\right) \circ u
$$

$$
\overline{\mathscr{A}}(u)(\bar{w}, \bar{w})=\int_{\partial u(\Omega)}\left|\nabla^{\top} w^{\perp}\right|^{2} d S
$$

for any $\bar{w} \in T_{u} \Gamma$ and $w=\bar{w} \circ u^{-1}$. In the Eulerian coordinates, $\overline{\mathscr{A}}$ takes the form

$$
\mathscr{A}(u)(w)=\nabla \mathcal{H}\left(-\Delta_{\partial u\left(\Omega_{0}\right)}\left(\left.w\right|_{\partial u\left(\Omega_{0}\right)}\right)^{\perp}\right), \quad \forall w: u\left(\Omega_{0}\right) \rightarrow \mathbb{R}^{n} \text { satisfying } \nabla \cdot w=0 .
$$

Since $\overline{\mathscr{D}}^{2} S(u)$ is self-adjoint, then

$$
\overline{\mathscr{D}}^{2} S(u)=\overline{\mathscr{A}}+\text { at most } 2 \text { nd order diff. operators }
$$


Computing $\overline{\mathscr{R}}$. In the linearized equation $(2.12)$, we need to calculate $\overline{\mathscr{R}}(u)\left(u_{t}, \bar{w}\right) u_{t}$ for a linearized solution $\bar{w}(t, \cdot)$. Therefore, we may again assume that $u(t, \cdot)$ is a sufficiently smooth critical path of the action $I$, thus $\bar{v} \triangleq v \circ u$ is smooth as well, and study the operator $\overline{\mathscr{R}}(u)\left(u_{t}, \cdot\right) u_{t}$ on $\bar{w}$.

Here we apply a well-known formula in Riemannain geometry formally. For any $\bar{v}, \bar{w} \in T_{u} \Gamma$, let $v=\bar{v} \circ u^{-1}$ and $w=\bar{w} \circ u^{-1}$

$$
\overline{\mathscr{R}}(u)(\bar{v}, \bar{w}) \bar{v} \cdot \bar{w}=I I_{u}(\bar{v}, \bar{v}) \cdot I I_{u}(\bar{w}, \bar{w})-I I_{u}(\bar{v}, \bar{w})^{2}=\int_{u\left(\Omega_{0}\right)} \nabla p_{v, v} \nabla p_{w, w}-\left|\nabla p_{v, w}\right|^{2} d x .
$$

For smooth $v$ and $w \in L^{2}\left(u\left(\Omega_{0}\right)\right)$, clearly $\left|\nabla p_{v, w}\right|_{L^{2}\left(u\left(\Omega_{0}\right)\right)} \leq C|w|_{L^{2}\left(u\left(\Omega_{t}\right)\right)}$. As for the term,

$$
\begin{aligned}
\int_{u\left(\Omega_{0}\right)} \nabla p_{v, v} \nabla p_{w, w} d x & =\int_{u\left(\Omega_{0}\right)} p_{v, v} \operatorname{tr}(D w)^{2} d x=\int_{u\left(\Omega_{0}\right)}-\nabla_{w} w \cdot \nabla p_{v, v} ; d x \\
& =\int_{\partial u\left(\Omega_{0}\right)}\left(-\nabla_{N} p_{v, v}\right)\left(w^{\perp}\right)^{2} d S+\int_{u\left(\Omega_{0}\right)} D^{2} p_{v, v}(w, w) d x .
\end{aligned}
$$

Much as in the derivation of (2.9), we derive an self-adjoint operator $\overline{\mathscr{R}}_{0}(v)$ on $T_{u} \Gamma$, depending on $u$ and $v$,

which satisfies

$$
\overline{\mathscr{R}}_{0}(v)(\bar{w})=\left(\nabla \mathcal{H}\left(-\nabla_{N} p_{v, v}\left(\left.w\right|_{\partial u\left(\Omega_{0}\right)}\right)^{\perp}\right)\right) \circ u
$$

$$
\overline{\mathscr{R}}_{0}(v)(\bar{w}, \bar{w})=\int_{\partial u(\Omega)}-\left.\nabla_{N} p_{v, v} w^{\perp}\right|^{2} d S .
$$

In the Eulerian coordinates, $\overline{\mathscr{R}}_{0}(v)$ takes the form

$$
\mathscr{R}_{0}(v)(w)=\nabla \mathcal{H}\left(-\nabla_{N} p_{v, v}\left(\left.w\right|_{\partial u\left(\Omega_{0}\right)}\right)^{\perp}\right), \quad \forall w: u\left(\Omega_{0}\right) \rightarrow \mathbb{R}^{n} \text { satisfying } \nabla \cdot w=0 .
$$

Therefore, in a very rough sense,

$$
\overline{\mathscr{R}}(u)(\bar{v}, \bar{w}) \bar{v}=\overline{\mathscr{R}}_{0}(\bar{v})+\text { bounded operators }
$$

where we used the fact that $<\overline{\mathscr{R}}(u)(\bar{v}, \cdot) \bar{v}, \cdot>$ is self-adjoint.

\section{The GeOMETRY OF EVOlVing DOMAins}

Suppose $\Omega_{t} \subset \mathbb{R}^{n}$ is a family of smooth domains with the parameter $t$, moving with a smooth velocity vector field $v(t, x), x \in \Omega_{t}$. We calculate various quantities related to the evolution of the geometry of the domain, which are essential in the energy estimate of the free boundary problem of the Euler's equations.

3.1. Material derivative $\mathbf{D}_{t}$. For any $x_{0} \in \bar{\Omega}_{t_{0}}$, the particle path $x(t)$ is the solution of the ODE:

$$
x_{t}=v(t, x) \quad x\left(t_{0}\right)=x_{0}
$$

and the material derivative $\mathbf{D}_{t}=\partial_{t}+\nabla_{v}$ is differentiation along the direction of $x(t)$ in the space time domain in $\mathbb{R}^{n} \times \mathbb{R}$. Clearly, $x(t) \in \partial \Omega_{t}$ if $x_{0} \in \partial \Omega_{t_{0}}$.

Calculations of $\mathbf{D}_{t} N$ and $\mathbf{D}_{t} S$. At any $x_{0} \in \partial \Omega_{t_{0}}, \mathbf{D}_{t} N\left(t_{0}, x_{0}\right) \perp N\left(t_{0}, x_{0}\right)$ since $|N(t, x)| \equiv 1$. To derive $\mathbf{D}_{t} N\left(t_{0}, x_{0}\right)$, let $\tau(t) \in T_{x(t)} \partial \Omega_{t}$ be a solution to the linearized particle path ODE:

$$
\mathbf{D}_{t} \tau=\nabla_{\tau} v \quad \tau\left(t_{0}\right)=\tau_{0} \in T_{x_{0}} \partial \Omega_{t_{0}} .
$$

At $\left(t_{0}, x_{0}\right), \mathbf{D}_{t} N \cdot \tau_{0}=\mathbf{D}_{t}(N \cdot \tau)-N \cdot \mathbf{D}_{t} \tau=-(D v)^{*}(N) \cdot \tau_{0}$. Therefore, we have

$$
\mathbf{D}_{t} N=-\left((D v)^{*}(N)\right)^{\top} \text {. }
$$

From standard calculations for hypersurfaces

$$
\mathbf{D}_{t} d S=\left(v^{\perp} \kappa+\mathcal{D} \cdot v^{\top}\right) d S .
$$


Covariant differentiation $\mathbf{D}_{t}^{\top}$. For the family of hypersurfaces $\partial \Omega_{t}$ with the velocity field $v$ we define parallel transport along the material line $x(t)$ as follows. Given a tangent vector $\tau_{0} \in T_{x_{0}} \partial \Omega_{t_{0}}$, let $\tau(t)$ be the solution of the following ODE:

$$
\mathbf{D}_{t} \tau \perp T_{x(t)} \partial \Omega_{t} \Leftrightarrow \mathbf{D}_{t} \tau=\left(\nabla_{\tau} v \cdot N\right) N, \quad \tau\left(t_{0}\right)=\tau_{0}
$$

It is easy to verify that $\tau(t) \in T_{x(t)} \partial \Omega_{t}$ and that this transport preserves the inner product.

A natural connection between $T \partial \Omega_{t} \subset \mathbb{R}^{n}$ for different $t$ along the materials lines is provided by the above parallel transport which induces the covariant differentiation $\mathbf{D}_{t}^{\top}$, the projection of $\mathbf{D}_{t}$ in $\mathbb{R}^{n}$ acting on $w \in T_{x(t)} \partial \Omega_{t} \subset \mathbb{R}^{n}$. This covariant differentiation induces the covariant differentiations of linear (multilinear) operators on tensor products of $T \partial \Omega_{t}$ and $T^{*} \partial \Omega$, which will also denoted by $\mathbf{D}_{t}^{\top}$.

Calculation of $\mathbf{D}_{t}^{\top} \Pi$ and $\mathbf{D}_{t} \kappa$. Given $\tau \in T_{x_{0}} \partial \Omega_{t_{0}}$, let $\tau(t)$ be its parallel transport along the material line $x(t)$ which enable us to compute

$$
\left(\mathbf{D}_{t}^{\top} \Pi\right)(\tau)=\mathbf{D}_{t}^{\top}(\Pi(\tau))=\left(\mathbf{D}_{t} \nabla_{\tau} N\right)^{\top}=\left(\nabla_{\tau} \mathbf{D}_{t} N+\nabla_{\left[\mathbf{D}_{t}, \tau\right]} N\right)^{\top}=\mathcal{D}_{\tau} \mathbf{D}_{t} N+\Pi\left(\left(\left[\mathbf{D}_{t}, \tau\right]\right)^{\top}\right)
$$

From (3.3) and (3.1), we have

$$
\begin{gathered}
{\left[\mathbf{D}_{t}, \tau\right]=\mathbf{D}_{t} \tau-\nabla_{\tau}\left(\frac{\partial}{\partial t}+v\right)=\left(\nabla_{\tau} v \cdot N\right) N-\nabla_{\tau} v=-\left(\nabla_{\tau} v\right)^{\top},} \\
\left(\mathbf{D}_{t}^{\top} \Pi\right)(\tau)=-\mathcal{D}_{\tau}\left(\left((D v)^{*}(N)\right)^{\top}\right)-\Pi\left(\left(\nabla_{\tau} v\right)^{\top}\right) .
\end{gathered}
$$

To calculate $\mathbf{D}_{t} \kappa$ at $\left(t_{0}, x_{0}\right)$, we take an orthonormal frame $\left\{\tau_{1}, \ldots, \tau_{n-1}\right\}$ of $T_{x_{0}} \partial \Omega_{t_{0}}$ and parallel transport it into an orthonormal frame along $x(t)$. Thus $\mathbf{D}_{t} \kappa=\mathbf{D}_{t}\left(\Pi\left(\tau_{i}\right) \cdot \tau_{i}\right)=\left(\mathbf{D}_{t}^{\top} \Pi\right)\left(\tau_{i}\right) \cdot \tau_{i}$ and (3.5) give slightly different but useful forms for $\mathbf{D}_{t} \kappa$

$$
\begin{aligned}
& \mathbf{D}_{t} \kappa=-\mathcal{D} \cdot\left((D v)^{*}(N)\right)^{\top}-\Pi\left(\tau_{i}\right) \cdot \nabla_{\tau_{i}} v=-\Delta_{\partial \Omega} v \cdot N-2 \Pi \cdot\left(\left(\left.D^{\top}\right|_{T \partial \Omega_{t}}\right) v\right) \\
& \mathbf{D}_{t} \kappa=-\Delta_{\partial \Omega} v^{\perp}-v^{\perp}|\Pi|^{2}+(\mathcal{D} \cdot \Pi)\left(v^{\top}\right) .
\end{aligned}
$$

Calculations of commutators involving $\mathbf{D}_{t}$. In the following, we will calculate the commutators of $\mathbf{D}_{t}$ with operators $\mathcal{H}, \mathcal{N}$, and $\Delta_{\partial \Omega}$, to show that they are of lower orders.

- $\left[\mathbf{D}_{t}, \mathcal{H}\right] f=\Delta^{-1}\left(2 D v \cdot D^{2} f_{\mathcal{H}}+\nabla f_{\mathcal{H}} \cdot \Delta v\right)$.

To start, we write the basic formula for any function $f(t, x), x \in \Omega_{t}$,

$$
\mathbf{D}_{t} \nabla f=\nabla \mathbf{D}_{t} f-(D v)^{*}(\nabla f) .
$$

For the tangential gradient, using $\nabla^{\top} f=\nabla f-\left(\nabla_{N} f\right) N$, it is straight forward to obtain

$$
\mathbf{D}_{t}^{\top} \nabla^{\top} f=\nabla^{\top} \mathbf{D}_{t} f-\left((D v)^{*}\left(\nabla^{\top} f\right)\right)^{\top}
$$

Let $f(t, x), x \in \partial \Omega_{t}$, be a smooth function. Recall $f_{\mathcal{H}}=\mathcal{H}(f)$ represents the harmonic extension of $f$ into $\Omega_{t}$. We have

$$
\Delta \mathbf{D}_{t} f_{\mathcal{H}}=\mathbf{D}_{t} \Delta f_{\mathcal{H}}+2 \nabla v \cdot D^{2} f_{\mathcal{H}}+\nabla_{\Delta v} f_{\mathcal{H}}=2 D v \cdot D^{2} f_{\mathcal{H}}+\nabla f_{\mathcal{H}} \cdot \Delta v
$$

which implies

$$
\mathbf{D}_{t} f_{\mathcal{H}}=\mathcal{H}\left(\mathbf{D}_{t} f\right)+\Delta^{-1} \Delta \mathbf{D}_{t} f_{\mathcal{H}}=\mathcal{H}\left(\mathbf{D}_{t} f\right)+\Delta^{-1}\left(2 D v \cdot D^{2} f_{\mathcal{H}}+\nabla f_{\mathcal{H}} \cdot \Delta v\right)
$$

Therefore we can write

$$
\mathbf{D}_{t} \mathcal{H}(f)=\mathcal{H}\left(\mathbf{D}_{t} f\right)+\Delta^{-1}\left(2 D v \cdot D^{2} f_{\mathcal{H}}+\nabla f_{\mathcal{H}} \cdot \Delta v\right) .
$$

- $\left[\mathbf{D}_{t}, \Delta^{-1}\right] g=\Delta^{-1}\left(2 D v \cdot D^{2} \Delta^{-1} g+\Delta v \cdot \nabla \Delta^{-1} g\right)$.

Next, we calculate $\left[\mathbf{D}_{t}, \Delta^{-1}\right]$. Let $g(t, x), x \in \Omega_{t}$ be a smooth function and $\phi=\Delta^{-1} g$. From the first half of (3.10) where $\Delta f=0$ was not used,

$$
\mathbf{D}_{t} g=\mathbf{D}_{t} \Delta \phi=\Delta \mathbf{D}_{t} \phi-2 D v \cdot D^{2} \phi-\Delta v \cdot \nabla \phi .
$$


Since $\left.\mathbf{D}_{t} \phi\right|_{\partial \Omega_{t}}=0$, we obtain

$$
\mathbf{D}_{t} \Delta^{-1} g=\Delta^{-1} \mathbf{D}_{t} g+\Delta^{-1}\left(2 D v \cdot D^{2} \Delta^{-1} g+\Delta v \cdot \nabla \Delta^{-1} g\right)
$$

- $\left[\mathbf{D}_{t}, \mathcal{N}\right] f=\nabla_{N} \Delta^{-1}\left(2 D v \cdot D^{2} f_{\mathcal{H}}+\nabla f_{\mathcal{H}} \cdot \Delta v\right)-\nabla f_{\mathcal{H}} \cdot \nabla_{N} v-\nabla_{\nabla^{\top} f} v \cdot N$.

To calculate the commutator of $\left[\mathbf{D}_{t}, \mathcal{N}\right]$, from (3.1), (3.8) and (3.11), we have

$$
\begin{aligned}
\mathbf{D}_{t}\left(\nabla f_{\mathcal{H}} \cdot N\right) & =\nabla_{N} \mathbf{D}_{t} f_{\mathcal{H}}-\nabla f_{\mathcal{H}} \cdot \nabla_{N} v+\nabla f_{\mathcal{H}} \cdot \mathbf{D}_{t} N \\
& =\nabla_{N}\left[\mathcal{H}\left(\mathbf{D}_{t} f\right)+\Delta^{-1}\left(2 D v \cdot D^{2} f_{\mathcal{H}}+\nabla f_{\mathcal{H}} \cdot \Delta v\right)\right]-\nabla f_{\mathcal{H}} \cdot \nabla_{N} v-\nabla_{\nabla^{\top} f} v \cdot N .
\end{aligned}
$$

Thus,

$$
\mathbf{D}_{t} \mathcal{N}(f)=\mathcal{N}\left(\mathbf{D}_{t} f\right)+\nabla_{N} \Delta^{-1}\left(2 D v \cdot D^{2} f_{\mathcal{H}}+\nabla f_{\mathcal{H}} \cdot \Delta v\right)-\nabla f_{\mathcal{H}} \cdot \nabla_{N} v-\nabla_{\nabla^{\top}} v \cdot N .
$$

- $\left[\Delta_{\partial \Omega_{t}}, \mathbf{D}_{t}\right] f=2 \mathcal{D}^{2} f \cdot\left(\left(\left.D^{\top}\right|_{T \partial \Omega_{t}}\right) v\right)+\nabla^{\top} f \cdot \Delta_{\partial \Omega_{t}} v-\kappa \nabla_{\nabla^{\top} f} v \cdot N$.

In order to calculate the commutator $\left[\Delta_{\partial \Omega_{t}}, \mathbf{D}_{t}\right]$ at $x_{0} \in \partial \Omega_{t_{0}}$, take an orthonormal frame $\left\{\tau_{1}, \ldots, \tau_{n-1}\right\}$ of $T_{x_{0}} \partial \Omega_{t}$. We first extend this to an orthonormal frame to $T_{x} \partial \Omega_{t_{0}}$ for all $x \in \partial \Omega_{t_{0}}$ close to $x_{0}$ by parallel transporting $\left\{\tau_{1}, \ldots, \tau_{n-1}\right\}$ along geodesics on $\partial \Omega_{t_{0}}$ starting from $x_{0}$. Parallel transporting them again along the material lines $x(t)$, we obtain an orthonormal frame $\left\{\tau_{1}, \ldots, \tau_{n-1}\right\}$ of $T_{x} \partial \Omega_{t}$ for all $(t, x)$ near $\left(t_{0}, x_{0}\right)$. From the standard Riemannian geometry, this orthonormal frame satisfies the property that, at $\left(t_{0}, x_{0}\right), \mathcal{D} \tau_{j}=0$ and $\left[\tau_{i}, \tau_{j}\right]=\mathcal{D}_{\tau_{i}} \tau_{j}-\mathcal{D}_{\tau_{j}} \tau_{i}=0$, which will be used repeatedly. For any smooth function $f(t, x)$ defined on $\partial \Omega_{t}$, at $\left(t_{0}, x_{0}\right)$,

$$
\begin{aligned}
\mathbf{D}_{t} \Delta_{\partial \Omega_{t}} f & =\mathbf{D}_{t}\left(\nabla_{\tau_{j}} \nabla_{\tau_{j}} f-\nabla_{\mathcal{D}_{\tau_{j}} \tau_{j}} f\right)=\nabla_{\tau_{j}} \mathbf{D}_{t} \nabla_{\tau_{j}} f+\nabla_{\left[\mathbf{D}_{t}, \tau_{j}\right]} \nabla_{\tau_{j}} f-\nabla_{\left[\mathbf{D}_{t}, \mathcal{D}_{\tau_{j}} \tau_{j}\right]} f \\
& =\nabla_{\tau_{j}} \nabla_{\tau_{j}} \mathbf{D}_{t} f+\nabla_{\tau_{j}} \nabla_{\left[\mathbf{D}_{t}, \tau_{j}\right]} f+\nabla_{\left[\mathbf{D}_{t}, \tau_{j}\right]} \nabla_{\tau_{j}} f-\nabla_{\left[\mathbf{D}_{t}, \mathcal{D}_{\tau_{j}} \tau_{j}\right]} f \\
& =\Delta_{\partial \Omega_{t}} \mathbf{D}_{t} f+2 \mathcal{D}^{2} f\left(\tau_{j},\left[\mathbf{D}_{t}, \tau_{j}\right]\right)+\nabla_{\mathcal{D}_{\tau_{j}}\left[\mathbf{D}_{t}, \tau_{j}\right]-\left[\mathbf{D}_{t}, \mathcal{D}_{\tau_{j}} \tau_{j}\right]} f .
\end{aligned}
$$

For any vector field $\tau(t, x) \in T_{x} \partial \Omega_{t}$, it is easy to see that $\left[\mathbf{D}_{t}, \tau\right] \in T_{x} \partial \Omega_{t}$ since (a) $\tau, \frac{\partial}{\partial t}+v \in$ $T\left(\cup_{t} \partial \Omega_{t}\right) \Rightarrow\left[\mathbf{D}_{t}, \tau\right] \in T\left(\cup_{t} \partial \Omega_{t}\right)$ and $(\mathrm{b})\left[\mathbf{D}_{t}, \tau\right]=\mathbf{D}_{t} \tau-\nabla_{\tau} v$ does not have $\frac{\partial}{\partial t}$ component. Thus, $\mathcal{D}_{\tau_{j}}\left[\mathbf{D}_{t}, \tau_{j}\right]-\left[\mathbf{D}_{t}, \mathcal{D}_{\tau_{j}} \tau_{j}\right] \in T \partial \Omega_{t}$ and we can drop all the normal components in its calculation. Using $\mathcal{D}_{\tau_{j}} \tau_{j}=\nabla_{\tau_{j}} \tau_{j}+\kappa N$ and $\mathcal{D} \tau_{j}=0$ at $\left(t_{0}, x_{0}\right)$, we obtain at $\left(t_{0}, x_{0}\right)$,

$$
\begin{aligned}
& \mathcal{D}_{\tau_{j}}\left[\mathbf{D}_{t}, \tau_{j}\right]-\left[\mathbf{D}_{t}, \mathcal{D}_{\tau_{j}} \tau_{j}\right]=\left(\nabla_{\tau_{j}}\left[\mathbf{D}_{t}, \tau_{j}\right]-\mathbf{D}_{t}\left(\nabla_{\tau_{j}} \tau_{j}+\kappa N\right)\right)^{\top} \\
= & \left.\left(\nabla_{\tau_{j}} \mathbf{D}_{t} \tau_{j}-\nabla_{\tau_{j}} \nabla_{\tau_{j}} v-\mathbf{D}_{t} \nabla_{\tau_{j}} \tau_{j}-\kappa \mathbf{D}_{t} N\right)^{\top}=-\left(\Delta_{\partial \Omega_{t}} v\right)^{\top}+\kappa\left((D v)^{*}(N)\right)\right)^{\top} .
\end{aligned}
$$

Therefore, from (3.4),

$$
\mathbf{D}_{t} \Delta_{\partial \Omega_{t}} f=\Delta_{\partial \Omega_{t}} \mathbf{D}_{t} f-2 \mathcal{D}^{2} f \cdot\left(\left(\left.D^{\top}\right|_{T \partial \Omega_{t}}\right) v\right)-\nabla^{\top} f \cdot \Delta_{\partial \Omega_{t}} v+\kappa \nabla_{\nabla^{\top} f} v \cdot N .
$$

Calculation of $\mathbf{D}_{t}^{2} \kappa$. This calculation starts with formula (3.6). Since $\Pi: T \partial \Omega_{t} \rightarrow T \partial \Omega_{t}$ then $\left.\Pi \cdot D^{\top}\right|_{T \partial \Omega_{t}} v=\left.\Pi \cdot \nabla\right|_{T \partial \Omega_{t}} v$. Let $\left\{\tau_{1}, \ldots, \tau_{n-1}\right\}$ be an orthonormal frame which is the parallel transport of an orthonormal frame $T_{x_{0}} \partial \Omega_{t_{0}}$ along the material line $x(t) \in \partial \Omega_{t}$. From (3.1), (3.5), (3.8), and (3.6), we have at $\left(t_{0}, x_{0}\right)$,

$$
\begin{aligned}
\mathbf{D}_{t}^{2} \kappa= & -\mathbf{D}_{t} \Delta_{\partial \Omega_{t}} v \cdot N-\Delta_{\partial \Omega_{t}} v \cdot \mathbf{D}_{t} N-2\left(\mathbf{D}_{t}^{\top}\left(\Pi\left(\tau_{i}\right)\right)\right) \cdot \nabla_{\tau_{i}} v-2 \Pi\left(\tau_{i}\right) \cdot \mathbf{D}_{t}\left(\nabla_{\tau_{i}} v\right) \\
= & -\mathbf{D}_{t} \Delta_{\partial \Omega_{t}} v \cdot N+\Delta_{\partial \Omega_{t}} v \cdot(D v)^{*}(N)^{\top}+2 \mathcal{D}_{\tau_{i}}\left(\left((D v)^{*}(N)\right)^{\top}\right) \cdot \nabla_{\tau_{i}} v \\
& +2 \Pi\left(\left(\nabla_{\tau_{i}} v\right)^{\top}\right) \cdot \nabla_{\tau_{i}} v-2 \Pi\left(\tau_{i}\right) \cdot \nabla_{\mathbf{D}_{t} \tau_{i}} v-2 \Pi\left(\tau_{i}\right) \cdot \nabla_{\tau_{i}} \mathbf{D}_{t} v+2 \Pi\left(\tau_{i}\right) \cdot(D v)^{2}\left(\tau_{i}\right) \\
= & -\mathbf{D}_{t} \Delta_{\partial \Omega_{t}} v \cdot N-2 \Pi \cdot\left(\left.D^{\top}\right|_{T \partial \Omega_{t_{0}}} \mathbf{D}_{t} v\right)+\Delta_{\partial \Omega_{t}} v \cdot(D v)^{*}(N)^{\top}+2\left[\mathcal{D}\left(\left((D v)^{*}(N)\right)^{\top}\right)\right. \\
& \left.+\Pi\left(\left(\left.D^{\top}\right|_{T \partial \Omega_{t_{0}}} v\right)^{\top}\right)\right] \cdot\left(\left.D^{\top}\right|_{T \partial \Omega_{t_{0}}} v\right)+2 \Pi \cdot\left(\left.(D v)^{2}\right|_{T \partial \Omega_{t}}\right)^{\top} .
\end{aligned}
$$

To compute $\mathbf{D}_{t} \Delta_{\partial \Omega_{t}} v \cdot N$ from (3.14) we need the general formula

$$
\mathcal{D}^{2} f\left(\tau, \tau^{\prime}\right)=D^{2} f\left(\tau, \tau^{\prime}\right)-\left(\Pi(\tau) \cdot \tau^{\prime}\right) \nabla_{N} f .
$$


for any $\tau, \tau^{\prime} \in T_{x_{0}} \partial \Omega_{t_{0}}$. Therefore,

$$
\begin{aligned}
-\mathbf{D}_{t} \Delta_{\partial \Omega_{t}} v \cdot N= & -N \cdot \Delta_{\partial \Omega_{t}} \mathbf{D}_{t} v+2 N \cdot D^{2} v\left(\tau_{i},\left(\nabla_{\tau_{i}} v\right)^{\top}\right)-2\left(\nabla_{N} v \cdot N\right)\left(\Pi \cdot\left(\left.D^{\top}\right|_{T \partial \Omega_{t_{0}}} v\right)\right) \\
& +N \cdot \nabla v\left(\left(\Delta_{\partial \Omega_{t}} v\right)^{\top}\right)-\kappa\left|(\nabla v)^{*}(N)^{\top}\right|^{2} .
\end{aligned}
$$

When $v$ and $\Omega_{t}$ satisfy the Euler's equation, the expression for $\mathbf{D}_{t}^{2} \kappa$ can be written as

$$
\mathbf{D}_{t}^{2} \kappa=-N \cdot \Delta_{\partial \Omega_{t}} \mathbf{D}_{t} v+2 \epsilon^{2} \Pi \cdot\left(\left.D^{\top}\right|_{T \partial \Omega} J\right)+r
$$

where we signaled out the important terms in the above equation

3.2. An important vector field for the water wave problem. Since $J=\nabla \kappa_{\mathcal{H}}$ appears in the Euler's equation as a force generated by the surface tension and its regularity is closely related to that of $\partial \Omega_{t}$, we will study the temporal evolution of $J$ for the rest of this section.

Computing $\mathbf{D}_{t} J$ and $\mathbf{D}_{t}^{2} J$. From (3.6), (3.8), (3.11) and the definition of $J$,

$$
\begin{aligned}
\mathbf{D}_{t} J= & \nabla \mathbf{D}_{t} \kappa_{\mathcal{H}}-(D v)^{*} J=\nabla \mathcal{H}\left(\mathbf{D}_{t} \kappa\right)+\nabla \Delta^{-1}[2 D v \cdot D J+J \cdot \Delta v]-(D v)^{*} J \\
= & \nabla \mathcal{H}\left(\Delta_{\partial \Omega_{t}} v \cdot N\right)-2 \nabla \mathcal{H}\left(\Pi \cdot\left(\left(\left.D^{\top}\right|_{T \partial \Omega_{t}}\right) v\right)\right) \\
& +\nabla \Delta^{-1}[2 D v \cdot D J+J \cdot \Delta v]-(D v)^{*} J .
\end{aligned}
$$

From (3.7), a slightly different way to write $\mathbf{D}_{t} J$ is

$$
\begin{array}{r}
\mathbf{D}_{t} J=-\nabla \mathcal{H}\left(\Delta_{\partial \Omega_{t}} v^{\perp}\right)+\nabla \mathcal{H}\left[-v^{\perp}|\Pi|^{2}+(\mathcal{D} \cdot \Pi)\left(v^{\top}\right)\right] \\
+\nabla \Delta^{-1}[2 D v \cdot D J+J \cdot \Delta v]-(D v)^{*} J
\end{array}
$$

Generally, when the surface tension is of order $O(1)$, it is sufficient to consider $\mathbf{D}_{t} J$. However, when there is no surface tension or the surface tension converges to 0 , we have to calculate $\mathbf{D}_{t}^{2} J$. Differentiating (3.18), we obtain

$$
\begin{aligned}
\mathbf{D}_{t}^{2} J & =\mathbf{D}_{t}\left(\nabla \mathbf{D}_{t} \kappa_{\mathcal{H}}-(D v)^{*} J\right) \\
& =\nabla \mathbf{D}_{t}^{2} \kappa_{\mathcal{H}}-(D v)^{*} \nabla \mathbf{D}_{t} \kappa_{\mathcal{H}}-(D v)^{*} \mathbf{D}_{t} J-\left(\mathbf{D}_{t} D v\right)^{*} J \\
& =\nabla \mathbf{D}_{t}^{2} \kappa_{\mathcal{H}}-2(D v)^{*} \mathbf{D}_{t} J-\left((D v)^{2}\right)^{*} J-\left(D \mathbf{D}_{t} v-(D v)^{2}\right)^{*} J .
\end{aligned}
$$

Since $\kappa_{\mathcal{H}}$ is harmonic, from (3.11) and (3.18),

$$
\begin{aligned}
\mathbf{D}_{t}^{2} \kappa_{\mathcal{H}}= & \mathbf{D}_{t}\left[\mathcal{H}\left(\mathbf{D}_{t} \kappa\right)+\Delta^{-1}(2 D v \cdot D J+J \cdot \Delta v)\right] \\
= & \mathcal{H}\left(\mathbf{D}_{t}^{2} \kappa\right)+\Delta^{-1}\left(2 D v \cdot D^{2}+\Delta v \cdot \nabla\right) \mathcal{H}\left(\mathbf{D}_{t} \kappa\right)+\mathbf{D}_{t} \Delta^{-1}(2 D v \cdot D J+J \cdot \Delta v) \\
= & \mathcal{H}\left(\mathbf{D}_{t}^{2} \kappa\right)+\Delta^{-1}(2 D v \cdot D+\Delta v \cdot)\left[\mathbf{D}_{t} J-\nabla \Delta^{-1}(2 D v \cdot D J+J \cdot \Delta v)-(D v)^{*} J\right] \\
& +\mathbf{D}_{t} \Delta^{-1}(2 D v \cdot D J+J \cdot \Delta v)
\end{aligned}
$$

A more explicit expression of $\mathcal{H}\left(\mathbf{D}_{t}^{2} \kappa\right)$ can be derived from (3.17). The $\mathbf{D}_{t} \Delta^{-1}$ is another term in the above equation that can be explicitly calculated from (3.12) and (3.10) to write

$$
\begin{aligned}
\mathbf{D}_{t} \Delta^{-1} & (2 D v \cdot D J+J \cdot \Delta v) \\
= & \Delta^{-1} \mathbf{D}_{t}(2 D v \cdot D J+J \cdot \Delta v)+\Delta^{-1}\left[\left(2 D v \cdot D^{2}+\Delta v \cdot \nabla\right) \Delta^{-1}(2 D v \cdot D J+J \cdot \Delta v)\right] \\
= & \Delta^{-1}\left[2\left(D \mathbf{D}_{t} v-(D v)^{2}\right) \cdot D J+2 D v \cdot\left(D \mathbf{D}_{t} J-D J D v\right)+\mathbf{D}_{t} J \cdot \Delta v\right. \\
& \left.+J \cdot\left(\Delta \mathbf{D}_{t} v-\nabla_{\Delta v} v-2 \partial_{i} v^{j} \partial_{i j} v\right)\right] \\
& +\Delta^{-1}\left[\left(2 D v \cdot D^{2}+\Delta v \cdot \nabla\right) \Delta^{-1}(2 D v \cdot D J+J \cdot \Delta v)\right]
\end{aligned}
$$


Computing $\mathscr{D}_{t} J$ and $\mathscr{D}_{t t} J$ with divergence free $v$. In the rest of this section, assume $\nabla \cdot v=0$. Given any vector field $w$ defined on $\Omega_{t}$ with $\nabla \cdot w=0$, let $\mathscr{D}_{t} w$ denote the divergence free part of $\mathbf{D}_{t} w$. It is easy to calculate that

$$
\mathscr{D}_{t} w=\mathbf{D}_{t} w+\nabla p_{v, w}, \quad-\Delta p_{v, w}=\operatorname{tr}(D v D w),\left.\quad p_{v, w}\right|_{\partial \Omega_{t}}=0 .
$$

As $J$ is divergence free, we will decompose material derivatives of $J$ into the divergence parts and gradient parts, i.e. we consider $\mathscr{D}_{t} J$ and $\mathscr{D}_{t t} J$, the covariant derivatives defined in (3.23). Then we have

$$
\mathscr{D}_{t} J=\mathbf{D}_{t} J+\nabla p_{v, J}
$$

For the second order derivative,

$$
\mathscr{D}_{t}^{2} J=\mathbf{D}_{t} \mathscr{D}_{t} J+\nabla p_{v, \mathscr{D}_{t} J}=\mathbf{D}_{t}^{2} J+\mathbf{D}_{t} \nabla p_{v, J}+\nabla p_{v, \mathscr{D}_{t} J}
$$

where $p_{v, J}$ and $p_{v, \mathscr{D}_{t} J}$ are defined as in (3.23). Using (3.8), (3.12)

$$
\begin{aligned}
\mathbf{D}_{t} \nabla p_{v, J}= & \nabla \mathbf{D}_{t} p_{v, J}-(D v)^{*} \nabla p_{v, J}=\nabla \mathbf{D}_{t} \Delta^{-1}(D v \cdot D J)-(D v)^{*} \nabla p_{v, J} \\
= & \nabla \Delta^{-1} \mathbf{D}_{t}(D v \cdot D J)+\nabla \Delta^{-1}\left(2 D v \cdot D^{2}+\Delta v \cdot D\right) p_{v, J}-(D v)^{*} \nabla p_{v, J} \\
= & \nabla \Delta^{-1}\left[\left(D \mathbf{D}_{t} v-(D v)^{2}\right) \cdot D J+D v \cdot\left(D \mathbf{D}_{t} J-D J D v\right)\right] \\
& +\nabla \Delta^{-1}\left(2 D v \cdot D^{2}+\Delta v \cdot D\right) p_{v, J}-(D v)^{*} \nabla p_{v, J} .
\end{aligned}
$$

Using the above calculations, we will show in Lemma 4.4 that $J$ satisfies the linearized Euler's equation with lower order terms. The estimates on $J$ and $\mathbf{D}_{t} J$ from the linearized Euler's Equation will imply the estimates on the geometry of the moving domain and the velocity fields.

\section{Main Results}

In this section, we will derive local energy estimates and prove convergence theorems. We show that solutions of (E) with boundary condition (BC) are locally bounded

$$
v(t, \cdot) \in H^{3 k}\left(\Omega_{t}\right) \quad \text { and } \quad \partial \Omega_{t} \in H^{s_{0}}, \quad s_{0}=3 k \text { or } 3 k+1 \text { for } \epsilon=0 \text { or }>0
$$

where $k$ is an integer satisfying $3 k>\frac{n}{2}+1$ (equivalently $3 k \geq \frac{n}{2}+\frac{3}{2}$ ). When $\epsilon>0$, this estimate is obtained without any additional assumption and it may depend on $\epsilon$. To derive a priori estimates independent of $\epsilon$, we assume the Rayleigh-Taylor sign condition (RT): $-\nabla_{N} p_{v, v}(t, x)>a>0 \quad x \in \partial \Omega_{t}$ for some constant $a$.

Definition of the energies and statements of the theorems. The conserved energy of the Euler's equation is given by

$$
E_{0}=E_{0}(\Omega, v)=\int_{\Omega} \frac{1}{2}|v|^{2} d x+\epsilon^{2} S(\Omega)
$$

where $S(\Omega)=\int_{\partial \Omega} d S$ is the surface area. Higher order energies are based on the linearized Euler flow and thus involve the differential operators $\mathscr{D}_{t}, \mathscr{A}$, and $\mathscr{R}_{0}(v)$.

Recall that, for any vector field $w \in H^{s}(\Omega)$ with $\nabla \cdot w=0, \mathscr{A}$ is given by

$$
\mathscr{A}(w)=-\nabla \mathcal{H} \Delta_{\partial \Omega} w^{\perp} .
$$

$\mathscr{A}$ is a semi-positive definite self-adjoint third order differential operator if $\left.w\right|_{\partial \Omega}$ is not smoother than $N \in H^{s_{0}-1}(\partial \Omega)$. In fact, it is positive definite acting on the irrotational part $w_{i r}$ of $w$, i.e. $w_{i r}=\nabla \mathcal{H} \mathcal{N}^{-1} w^{\perp}$, see (6.20) for details.

Also recall that $\mathscr{R}_{0}(v)$, which depends on $\Omega$ as well as on a vector field $v \in H^{3 k}(\Omega)$ with $\nabla \cdot v=0$, is given by

$$
\mathscr{R}_{0}(v)(w)=\nabla \mathcal{H}\left(\left(-\nabla_{N} p_{v, v}\right) w^{\perp}\right)=\nabla \mathcal{H}\left(\left(-\nabla_{N} \Delta^{-1} \operatorname{tr}(D v D v)\right) w^{\perp}\right)
$$


By Lemma $6.4, \nabla_{N} p_{v, v} \in H^{3 k-\frac{3}{2}}(\partial \Omega)$ and therefore $\mathscr{R}_{0}(v)$ is a first order self-adjoint differential operator if $w$ is not smoother than $\nabla p_{v, v}$. Under the sign assumption (RT), $\mathscr{R}_{0}(v)$ is semi-positive definite, and like $\mathscr{A}$, it is positive definite on the irrotational part $w_{i r}$ of $w$.

Let $\omega_{v}: \mathbb{R}^{n} \rightarrow \mathbb{R}^{n}$, often simply written as $\omega$ for short, represent the curl or vorticity of a vector field $v$ defined on $\Omega$, i.e.

$$
\omega(X) \cdot Y=\nabla_{X} v \cdot Y-\nabla_{Y} v \cdot X
$$

for any vector $X, Y \in \mathbb{R}^{n}$. Viewing $\omega$ as a matrix, its entries are $\omega_{i}^{j}=\omega\left(\frac{\partial}{\partial x^{i}}\right) \cdot \frac{\partial}{\partial x^{j}}=\partial_{i} v^{j}-\partial_{j} v^{i}$.

Definition 4.1. For any domain $\Omega$ in $H^{s_{0}}, s_{0}=3 k$ if $\epsilon=0$ or $s_{0}=3 k+1$ if $\epsilon>0$, and any vector field $v \in H^{3 k}(\Omega)$ with $\nabla \cdot v=0$, define the energies $E(\Omega, v)$ and $E_{R T}(\Omega, v)$, often written as $E$ and $E_{R T}$ for short,

$$
E=\int_{\Omega} \frac{1}{2}\left|\mathscr{A}^{k-1} \mathscr{D}_{t} J\right|^{2}+\frac{\epsilon^{2}}{2}\left|\mathscr{A}^{k-\frac{1}{2}} J\right|^{2} d x+|\omega|_{H^{3 k-1}(\Omega)}^{2}, \quad E_{R T}=\int_{\Omega} \frac{1}{2} \mathscr{R}_{0}(v) \mathscr{A}^{k-1} J \cdot \mathscr{A}^{k-1} J d x
$$

where $\mathscr{D}_{t}$ is the divergence free part of $\mathbf{D}_{t} J$ defined in (3.23),

$$
\mathscr{D}_{t} J=\mathbf{D}_{t} J+\nabla p_{v, J}=\mathbf{D}_{t} J-\nabla \Delta^{-1} \operatorname{tr}(D v D J)
$$

Set $\mathcal{E}=E+E_{R T}$.

To estimate terms in the energy we need to consider the following type of $H^{s_{0}}$ neighborhoods of $\Omega_{*}$, a bounded connected domain in $\mathbb{R}^{n}$, which are bounded in $H^{s}$ for some $s \geq s_{0}$.

Definition 4.2. Let $\Lambda=\Lambda\left(\Omega_{*}, s_{0}, s, L, \delta\right)$ be the collection of all domains $\Omega$ satisfying

(A1) there exists a diffeomorphism $F: \partial \Omega_{*} \rightarrow \partial \Omega \subset \mathbb{R}^{n}$, so that $\left|F-i d_{\partial \Omega_{*}}\right|_{H^{s_{0}}\left(\partial \Omega_{*}\right)}<\delta$;

(A2) the mean curvature $\kappa$ of $\partial \Omega$ satisfies $|\kappa|_{H^{s-2}(\partial \Omega)}<L$.

Fix $0<\delta \ll 1$ and let $L_{0}=1+2|\kappa(0, \cdot)|_{H^{3 k-\frac{5}{2}}(\partial \Omega)}$ and $\Lambda_{0} \triangleq \Lambda\left(\Omega_{0}, 3 k-\frac{1}{2}, 3 k-\frac{1}{2}, L_{0}, \delta\right)$. By Lemma 6.4, and equations (6.10) and (6.19) we have

$$
\begin{array}{ll}
|\mathscr{A}|_{L\left(H^{s}(\Omega), H^{s-3}(\Omega)\right)} \leq C, & s \in[3,3 k-1] \\
\left|\mathscr{R}_{0}(v)\right|_{L\left(H^{s}(\Omega), H^{s-1}(\Omega)\right)} \leq C\left|\nabla_{N} p_{v, v}\right|_{H^{3 k-\frac{3}{2}(\partial \Omega)}} \leq C|v|_{H^{3 k}(\Omega)}^{2}, & s \in[1,3 k-1]
\end{array}
$$

$$
\left|p_{v, v}\right|_{H^{3 k}(\Omega)} \leq C\left|(D v)^{2}\right|_{H^{3 k-2}(\Omega)} \leq C|v|_{H^{3 k-\frac{5}{8}(\Omega)}}^{2}
$$

by Sobolev inequalities

where $C$ is uniform in $\Omega \in \Lambda_{0}$. Here used the fact $3 k \geq \frac{n}{2}+\frac{3}{2}$. The norm $H^{3 k-\frac{5}{8}}$ in (4.6) is chosen for convenience; any norm $H^{3 k-1+\alpha}$ would work with $\alpha>\frac{1}{4}$. The next proposition gives bounds on the velocity and mean curvature in terms of these energies.

Proposition 4.1. For $\Omega \in \Lambda_{0}$ with $\partial \Omega \in H^{s_{0}}$, we have

$$
\epsilon^{2}|\kappa|_{H^{3 k-1}(\partial \Omega)}^{2} \leq 3 E+C_{0} \epsilon^{2}, \quad|v|_{H^{3 k}(\Omega)}^{2} \leq C_{0}\left(E+E_{0}\right)
$$

and, if we also assume (RT),

$$
|\kappa|_{H^{3 k-2}(\partial \Omega)}^{2} \leq C_{*} E_{R T}+C_{0},
$$

for some constant $C_{*}, C_{0}>0 . C_{*}$ depends only on a in assumption (RT) and $C_{0}$ depends only on the set $\Lambda_{0}$.

The proof of this proposition will be given below. Using this result we will prove the following three theorems. The first theorem holds when there is surface tension which makes the regularity of $\partial \Omega_{t}$ better (in $H^{3 k+1}$ ) but the bound on $|\kappa|_{H^{3 k-1}\left(\partial \Omega_{t}\right)}$ depends on $\epsilon$, the strength of of the surface tension. 
Theorem 4.1. Assume $\epsilon>0$ and fix $\delta>0$ sufficiently small. Then there exists $L_{\epsilon}>0$ such that, if a solution of (E) and (BC) is given by $\Omega_{t}$ with $\partial \Omega_{t} \in H^{3 k+1}$ and $v(t, \cdot) \in C\left(H^{3 k}\left(\Omega_{t}\right)\right)$, then there exists $t^{*}>0$, depending only on $|v(0, \cdot)|_{H^{3 k}\left(\Omega_{t}\right)}, L_{\epsilon}$, and the set $\Lambda_{0}$, such that, for all $t \in\left[0, t^{*}\right]$,

$$
\begin{aligned}
& \Omega_{t} \in \Lambda_{0} \quad \text { and } \quad|\kappa|_{H^{3 k-1}\left(\partial \Omega_{t}\right)} \leq L_{\epsilon}, \\
& E\left(\Omega_{t}, v(t, \cdot)\right) \leq 2 E\left(\Omega_{0}, v(0, \cdot)\right)+C_{1}+\int_{0}^{t} P_{\epsilon}\left(E_{0}, E\left(\Omega_{t^{\prime}}, v\left(t^{\prime}, \cdot\right)\right)\right) d t^{\prime}
\end{aligned}
$$

where $P_{\epsilon}(\cdot)$ is a polynomial of positive coefficients determined only by $\epsilon$ and the set $\Lambda_{0}$ and $C_{\epsilon}$ is an constant determined only by $\epsilon,|v(0, \cdot)|_{H^{3 k-\frac{3}{2}}\left(\Omega_{0}\right)}$, and the set $\Lambda_{0}$.

Since the domain is evolving, the above continuity assumption of $v$ in $t$ means that there exists an extension of $v$ to $[0, T] \times \mathbb{R}^{n}$ which is continuous in $H^{3 k}\left(\mathbb{R}^{n}\right)$. The second theorem holds under the assumption (RT) and the estimates are uniform in $\epsilon \in[0,1]$. As it does not take the advantage of the surface tension even if it is present, the bound on the regularity of $\partial \Omega_{t}$ is only in $H^{3 k}$.

Theorem 4.2. Assume $\epsilon \in[0,1]$ and $(R T)$ holds. Fix sufficiently small $\delta>0$. There exists $L>0$ such that, if a solution of $(E)$ and $(B C)$ is given by $\Omega_{t}$ with $\partial \Omega_{t} \in H^{3 k}$ and $v(t, \cdot) \in C\left(H^{3 k}\left(\Omega_{t}\right)\right)$, then there exists $t^{*}>0$, depending only on $|v(0, \cdot)|_{H^{3 k}\left(\Omega_{t}\right)}$, L, and the set $\Lambda_{0}$, such that, for all $t \in\left[0, t^{*}\right]$,

$$
\begin{aligned}
& \Omega_{t} \in \Lambda_{0} \quad \text { and } \quad|\kappa|_{H^{3 k-2}\left(\partial \Omega_{t}\right)} \leq L, \\
& \mathcal{E}\left(\Omega_{t}, v(t, \cdot)\right) \leq \mathcal{E}\left(\Omega_{0}, v(0, \cdot)\right)+\int_{0}^{t} P\left(\mathcal{E}\left(\Omega_{t^{\prime}}, v\left(t^{\prime}, \cdot\right)\right)\right) d t^{\prime}
\end{aligned}
$$

where $P(\cdot)$ is a polynomial of positive coefficients uniform in $\epsilon$, determined by the set $\Lambda_{0}$.

An immediate consequence of the above theorem is convergence of solution as the surface tension approaches 0 .

Theorem 4.3. Assume (RT) holds. Fix the initial data $\partial \Omega_{0} \in H^{3 k+1}$ and $v(0, \cdot) \in H^{3 k}\left(\Omega_{0}\right)$. As $\epsilon \rightarrow 0$, subject to a subsequence, the solution of $(E)$ and $(B C)$ with vanishing surface tension converges to a solution of $(E)$ and $(B C)$ for $\epsilon=0$ weakly in the space of $\partial \Omega_{t} \in H^{3 k}$ and $v(t, \cdot) \in H^{3 k}$.

The above convergence of $\partial \Omega_{t}$ is in the sense of local coordinates and the convergence of $v$ can be obtained by using the Lagrangian coordinates $u(t, y)$ which is also in $H^{3 k}$. We also observe that the neighborhood $\Lambda_{0}$ of the domains $\Omega$ does not have to be centered at $\Omega_{0}$. Thus, since the constants involved in the energy estimates only depend on the neighborhoods and the norm of the initial velocity, these estimates provide a basis for a continuation argument local in time.

Proof of Proposition 4.1. From the definition of $\mathscr{A}$ and $\mathscr{R}_{0}(v)$, it is easy to obtain

$$
\begin{aligned}
& \epsilon^{2}\left|\nabla^{\top} \mathcal{N}\left(-\Delta_{\partial \Omega} \mathcal{N}\right)^{k-1} \kappa\right|_{L^{2}(\partial \Omega)}^{2}=\epsilon^{2}\left|\mathscr{A}^{k-\frac{1}{2}} J\right|_{L^{2}(\Omega)} \leq 2 E \\
& \int_{\partial \Omega}-\nabla_{N} p_{v, v}\left|\mathcal{N}\left(-\Delta_{\partial \Omega} \mathcal{N}\right)^{k-1} \kappa\right|^{2} d S=<\mathscr{R}_{0}(v) \mathscr{A}^{k-1} J, \mathscr{A}^{k-1} J>_{L^{2}(\Omega)}^{2}=2 E_{R T} .
\end{aligned}
$$

To estimate $\kappa$ in either $H^{3 k-2}(\partial \Omega)$ or $H^{3 k-1}(\partial \Omega)$, it is sufficient to use the estimates on functions and operators defined on $\partial \Omega$ considered as in $H^{3 k-\frac{1}{2}}$ only. Therefore, the inequality for $\kappa$ in Proposition 4.1 follows from (RT) and the fact that $\mathcal{N}$ behaves as a first order derivative (Theorem 6.1, (6.16), and (6.15)).

To estimate $v$, it is easy to calculate that

$$
\Delta v^{i}=\partial_{j} \omega_{j}^{i}
$$

which is part of the energy. Therefore, we only need to show that some boundary data of $v$ is controlled by $E$ and the conserved energy $E_{0}$. This boundary data of $v$ turns out to be $\nabla_{N} v$. Since 
$\nabla_{N} v=(D v)^{*}(N)+\omega(N)$ and $\omega$ is controlled by $E$, it suffices to estimate $\nu=(D v)^{*}(N)$.

Step 1. Tangential curl $\omega_{\nu}^{\top}$ of $\nu^{\top}$. Let $\nu^{\top}$ be the tangential component of $\nu$ and $\omega_{\nu}^{\top}(x): T_{x} \partial \Omega \rightarrow$ $T_{x} \partial \Omega$ be defined as

$$
\omega_{\nu}^{\top}(x)(X) \cdot Y=\mathcal{D}_{X} \nu^{\top} \cdot Y-\mathcal{D}_{Y} \nu^{\top} \cdot X=\nabla_{X} \nu^{\top} \cdot Y-\nabla_{Y} \nu^{\top} \cdot X
$$

for any $x \in \partial \Omega$ and $X, Y \in T_{x} \partial \Omega$. To obtain a more explicit form of $\omega_{\nu}^{\top}$, let $X$ and $Y$ be extended to tangent vector fields on a neighborhood of $x$ on $\partial \Omega$ by parallel transport along geodesics on $\partial \Omega$ emitting from $x$. From the definition of $\nu$, we have

$$
\begin{aligned}
\omega_{\nu}^{\top}(X) \cdot Y & =\nabla_{X}\left(\nabla_{Y} v \cdot N\right)-\mathcal{D}_{X} Y \cdot \nu-\nabla_{Y}\left(\nabla_{X} v \cdot N\right)+\mathcal{D}_{Y} X \cdot \nu \\
& =\Pi(X) \cdot \nabla_{Y} v-\Pi(Y) \cdot \nabla_{X} v .
\end{aligned}
$$

Therefore, by Sobolev inequalities, there exists $C>0$ uniform in $\Omega \in \Lambda_{0}$ so that

$$
\left|\omega_{\nu}^{\top}\right|_{H^{3 k-\frac{5}{2}}(\partial \Omega)} \leq C\left|\Pi \circ D^{\top} v\right|_{H^{3 k-\frac{5}{2}}(\partial \Omega)} \leq C|v|_{H^{3 k-\frac{1}{8}}(\Omega)}
$$

since $3 k \geq \frac{n}{2}+\frac{3}{2}$. Again here the norm $H^{3 k-\frac{1}{8}}$ is chosen to illustrate that the term is lower order. In fact any $H^{3 k-\alpha}$ with $0<\alpha<\frac{1}{4}$ works.

Step 2. Divergence $\mathcal{D} \cdot \nu^{\top}$. At any $x_{0} \in \partial \Omega$, let $\left\{X_{1}, \ldots, X_{n-1}\right\}$ be an orthonormal frame of $T_{x_{0}} \partial \Omega$. We extend them to orthonormal frames of $T_{x} \partial \Omega$ at each $x$ in a neighborhood of $x_{0}$ in $\partial \Omega$ by parallel transport along geodesics on $\partial \Omega$ emitting from $x_{0}$. At $x_{0}$,

$$
\mathcal{D} \cdot \nu^{\top}=\mathcal{D}_{X_{i}} \nu^{\top} \cdot X_{i}=\mathcal{D}_{X_{i}}\left(\nabla_{X_{i}} v \cdot N\right)=\Delta_{\partial \Omega} v \cdot N+\left(\left.D^{\top}\right|_{T \partial \Omega_{t}}\right) v \cdot \Pi .
$$

To control the first term on the right side, we use (3.24) and (3.18) to obtain

$$
\left|\mathscr{D}_{t} J+\nabla \mathcal{H}\left(\Delta_{\partial \Omega} v \cdot N\right)\right|_{H^{3 k-3}(\Omega)} \leq C|v|_{H^{3 k-\frac{1}{8}(\Omega)}}
$$

where we also used Lemma 6.4 for the estimates. To get boundary estimates, note that $\mathscr{D}_{t} J \cdot N$ and $N \cdot \nabla \mathcal{H}\left(\Delta_{\partial \Omega} v \cdot N\right)$ are well defined in $H^{3 k-\frac{7}{2}}(\partial \Omega)$ even if $k=1$ since they are divergence free. and thus combining the above inequality with the identity for $\mathcal{D} \cdot \nu^{\top}(4.11)$, we obtain

$$
\left|\mathcal{N}\left(\mathcal{D} \cdot \nu^{\top}\right)+\left(\mathscr{D}_{t} J\right)^{\perp}\right|_{H^{3 k-\frac{7}{2}}(\partial \Omega)} \leq C|v|_{H^{3 k-\frac{1}{8}(\Omega)}} \Rightarrow|\mathcal{D} \cdot \nu|_{H^{3 k-\frac{5}{2}(\partial \Omega)}}^{2} \leq C\left(E+|v|_{H^{3 k-\frac{1}{8}(\Omega)}}^{2}\right) .
$$

Step 3. Control of $\nu^{\top}$. Using the same frame as in Step 2, at $x_{0}$,

$$
\begin{aligned}
\Delta_{\partial \Omega} \nu^{\top} \cdot X_{i} & =\mathcal{D}_{X_{j}} \mathcal{D}_{X_{j}} \nu^{\top} \cdot X_{i}=\nabla_{X_{j}}\left(\mathcal{D}_{X_{j}} \nu^{\top} \cdot X_{i}\right)=\nabla_{X_{j}}\left(\mathcal{D}_{X_{i}} \nu^{\top} \cdot X_{j}+\omega_{\nu}^{\top}\left(X_{j}\right) \cdot X_{i}\right) \\
& =\nabla_{X_{i}}\left(\mathcal{D} \cdot \nu^{\top}\right)+\mathcal{R}\left(X_{i}, X_{j}\right) \nu^{\top} \cdot X_{j}+\left(\mathcal{D}_{X_{j}} \omega_{\nu}^{\top}\right)\left(X_{j}\right) \cdot X_{i} .
\end{aligned}
$$

One can also write

$$
\Delta_{\partial \Omega} \nu^{\top}=\nabla^{\top}\left(\mathcal{D} \cdot \nu^{\top}\right)+\operatorname{Ric}\left((\nabla v)^{*}(N)^{\top}\right)+\left(\mathcal{D}_{X_{j}} \omega_{\nu}^{\top}\right)\left(X_{j}\right)
$$

where Ric is the Ricci curvature of $\partial \Omega$. From the estimate on $\omega_{\nu}^{\top}$ and $\mathcal{D} \cdot \nu^{\top}$,

$$
\left|\nu^{\top}\right|_{H^{3 k-\frac{3}{2}}(\partial \Omega)}^{2} \leq C\left(E+|v|_{H^{3 k-\frac{1}{8}(\Omega)}}^{2}\right)
$$

with a uniform constant $C>0$.

Step 4. Normal component $\nu^{\perp}=\nabla_{N} v \cdot N$ of $\nu$. This will be estimated by calculating the divergence of $\nu$ in two ways. Recall $N_{\mathcal{H}}=\left(N_{\mathcal{H}}^{1}, \ldots, N_{\mathcal{H}}^{n}\right)$ denotes the harmonic extension of $N$ into $\Omega$. Let $\nu$ also be extended to $(D v)^{*}\left(N_{\mathcal{H}}\right)$. Near any $x_{0} \in \partial \Omega$, let $\left\{X_{1}, \ldots, X_{n-1}\right\}$ be the orthonormal frame of $T_{x} \partial \Omega$ constructed above and let $X_{n}=N_{\mathcal{H}}$. On one hand, at $x_{0}$,

$$
\begin{aligned}
\nabla \cdot \nu & =\nabla_{X_{i}}\left((D v)^{*}\left(N_{\mathcal{H}}\right)\right) \cdot X_{i}=\nabla_{X_{i}}\left(\nabla_{X_{i}} v \cdot N_{\mathcal{H}}\right)-N \cdot D v\left(\nabla_{X_{i}} X_{i}\right) \\
& =\left(\nabla_{X_{i}} \omega\right)\left(X_{i}\right) \cdot N+D v \cdot D N_{\mathcal{H}}
\end{aligned}
$$


where the first term in the last line follows from (4.9). Therefore

$$
\left|\nabla \cdot \nu-\left(\nabla_{X_{i}} \omega\right)\left(X_{i}\right) \cdot N\right|_{H^{3 k-\frac{5}{2}(\partial \Omega)}} \leq C|v|_{H^{3 k-\frac{1}{8}(\Omega)}} \cdot
$$

On the other hand, by decomposing $\nu$ into the tangential and normal parts, one may calculate $\nabla \cdot \nu$ alternatively

$$
\nabla \cdot \nu=\mathcal{D} \cdot \nu^{\top}+\kappa \nu^{\perp}+\nabla_{N} \nu \cdot N=\mathcal{D} \cdot \nu^{\top}+\kappa \nu^{\perp}+\nabla_{N}\left(\nabla_{N_{\mathcal{H}}} v \cdot N_{\mathcal{H}}\right)-N \cdot D v(\mathcal{N}(N))
$$

which along with the previous identity implies

$$
\left|\mathcal{D} \cdot \nu^{\top}+\nabla_{N}\left(\nabla_{N_{\mathcal{H}}} v \cdot N_{\mathcal{H}}\right)-\left(\nabla_{X_{i}} \omega\right)\left(X_{i}\right) \cdot N\right|_{H^{3 k-\frac{5}{2}}(\partial \Omega)} \leq C|v|_{H^{3 k-\frac{1}{8}(\Omega)}} \cdot
$$

Since $\omega$ is controlled by $E$ and $\mathcal{D} \cdot \nu^{\top}$ has been estimated in Step 2, we have

$$
\left|\nabla_{N}\left(\nabla_{N_{\mathcal{H}}} v \cdot N_{\mathcal{H}}\right)\right|_{\dot{H}^{3 k-\frac{5}{2}}(\partial \Omega)}^{2} \leq C\left(E+|v|_{H^{3 k-\frac{1}{8}(\Omega)}}^{2}\right)
$$

The following decomposition trick on $\partial \Omega$ has been used many times in the basic estimates in Sections 6 and 3 ,

$$
\begin{aligned}
\nabla_{N}\left(\nabla_{N_{\mathcal{H}}} v \cdot N_{\mathcal{H}}\right)= & \mathcal{N}\left(\nabla_{N} v \cdot N\right)+\nabla_{N} \Delta^{-1}\left(\nabla_{N_{\mathcal{H}}} \Delta v \cdot N_{\mathcal{H}}+2 \operatorname{tr}\left(\left(D N_{\mathcal{H}}\right)^{*} D v D N_{\mathcal{H}}\right)\right. \\
& \left.+2 D^{2} v\left(N_{\mathcal{H}}\right) \cdot D N_{\mathcal{H}}+2 D^{2} v\left(\frac{\partial N_{\mathcal{H}}}{\partial x^{i}}, \frac{\partial}{\partial x^{i}}\right) \cdot N_{\mathcal{H}}\right) .
\end{aligned}
$$

Using (4.9) again, we have with a uniform $C>0$,

$$
\left|\mathcal{N}\left(\nu^{\perp}\right)\right|_{H^{3 k-\frac{5}{2}(\partial \Omega)}}^{2}=\left|\mathcal{N}\left(\nabla_{N} v \cdot N\right)\right|_{\dot{H}^{3 k-\frac{5}{2}(\partial \Omega)}}^{2} \leq C\left(E+|v|_{H^{3 k-\frac{1}{8}(\Omega)}}^{2}\right)
$$

From Step 3 and Step 4 above, we have

$$
|\nu|_{\dot{H}^{3 k-\frac{3}{2}}(\partial \Omega)}^{2} \leq C\left(E+|v|_{H^{3 k-\frac{1}{8}(\Omega)}}^{2}\right)
$$

which implies the same estimate of the boundary data $\nabla_{N} v$ on $\partial \Omega$. Combining it with the Poisson equation (4.9), we obtain

$$
|v|_{H^{3 k}(\Omega)}^{2} \leq C\left(E+|v|_{H^{3 k-\frac{1}{8}(\Omega)}}^{2}\right) .
$$

Since $|v|_{H^{3 k-\frac{1}{8}(\Omega)}} \leq \beta|v|_{H^{3 k}(\Omega)}+C_{\beta}|v|_{L^{2}(\Omega)}$, proposition 4.1 follows immediately.

In the proof of Theorem 4.1 and Theorem 4.2, we will need the following lemmas.

Lemma 4.2. For any $\Omega \in \Lambda_{0}$ with $\kappa \in H^{s}(\partial \Omega), s \in\left[3 k-\frac{5}{2}, 3 k-\frac{3}{2}\right]$, we have

$$
|\Pi|_{H^{s}\left(\partial \Omega_{t}\right)}+|N|_{H^{s+1}\left(\partial \Omega_{t}\right)} \leq C\left(1+|\kappa|_{H^{s}\left(\partial \Omega_{t}\right)}\right)
$$

for some $C>0$ uniform in $\Omega \in \Lambda_{0}$.

Proof. We only need to prove the estimates for $\Pi$. This is obvious if $n=2$. For $n \geq 3$, we use identity (6.5):

Since $\Omega \in \Lambda_{0}$, we have

$$
-\Delta_{\partial \Omega} \Pi=-\mathcal{D}^{2} \kappa+\left(|\Pi|^{2} I-\kappa \Pi\right) \Pi \text {. }
$$

and it implies

$$
|\Pi|_{H^{3 k-\frac{5}{2}}\left(\partial \Omega_{t}\right)}+|\kappa|_{H^{3 k-\frac{5}{2}}\left(\partial \Omega_{t}\right)} \leq C
$$

$$
\left.|| \Pi\right|^{2} I-\left.\kappa \Pi\right|_{H^{s_{1}\left(\partial \Omega_{t}\right)}} \leq C, \quad \text { where } \quad s_{1}=\min \left\{6 k-5-\frac{n-1}{2}, 3 k-\frac{5}{2}\right\}
$$

as long as $3 k-\frac{5}{2} \neq \frac{n-1}{2}$. Therefore, for $s_{2} \in\left[3 k-\frac{5}{2}, 3 k-\frac{3}{2}\right] \backslash\left\{\frac{n-1}{2}\right\}$,

$$
\left|\left(|\Pi|^{2} I-\kappa \Pi\right) \Pi\right|_{H^{s_{3}\left(\partial \Omega_{t}\right)}} \leq C|\Pi|_{H^{s_{2}}\left(\partial \Omega_{t}\right)} \quad s_{3}=\min \left\{s_{1}, s_{1}+s_{2}-\frac{n-1}{2}\right\} .
$$


Since $s_{3}+2-s_{2} \geq \min \left\{1,3 k-1-\frac{n}{2}\right\}>0$ and $s_{1}+2>3 k-\frac{3}{2}$, the estimate of $\Pi$ can be improved to $H^{s}\left(\partial \Omega_{t}\right)$ by bootstrap on $\Delta_{\partial \Omega_{t}}^{-1}$. The exceptional cases of the indices can be handled similarly.

Although this regularity of $\Pi$ in terms of $\kappa$ follows directly from Proposition 6.3, the point of this lemma is that the constant $C>0$ depends only on $\Lambda_{0}$, i.e., $\Omega \in H^{3 k-\frac{1}{2}}$. This is also the point of the following lemma.

Lemma 4.3. For $\Omega \in \Lambda_{0}$ with $\partial \Omega \in H^{3 k}$ and $v \in H^{3 k}(\Omega)$, we have

$$
\left|\nabla_{N_{\mathcal{H}}} p_{v, v}\right|_{H^{3 k-\frac{1}{2}(\Omega)}}+\left|D^{2} p_{v, v}\right|_{H^{3 k-\frac{3}{2}(\Omega)}} \leq C\left(1+|\kappa|_{H^{3 k-2}(\partial \Omega)}\right)|v|_{H^{3 k}(\Omega)}^{2} .
$$

for some $C>0$ uniform in $\Omega \in \Lambda_{0}$.

Proof. The idea of the proof is to use $\left.p_{v, v}\right|_{\partial \Omega_{0}}=0$ and the identity $f=\mathcal{H}\left(\left.f\right|_{\partial \Omega}\right)+\Delta^{-1} \Delta f$ for any $f: \Omega \rightarrow \mathbb{R}$. On the one hand, notice on $\partial \Omega$,

$$
\nabla_{N} \nabla_{N_{\mathcal{H}}} p_{v, v}=\mathcal{N}(N) \cdot \nabla p_{v, v}+D^{2} p_{v, v}(N, N)=\mathcal{N}(N) \cdot \nabla p_{v, v}+\Delta p_{v, v}-\kappa \nabla_{N} p_{v, v},
$$

which, along Lemma 4.2 and (4.6), implies

$$
\left|\nabla_{N} \nabla_{N_{\mathcal{H}}} p_{v, v}\right|_{H^{3 k-2}(\partial \Omega)} \leq C\left(1+|\kappa|_{H^{3 k-2}(\partial \Omega)}\right)|v|_{H^{3 k}(\Omega)}^{2} .
$$

On the other hand, using Lemma 4.2 and (4.6) as well,

$$
\begin{aligned}
\left|\Delta \nabla_{N_{\mathcal{H}}} p_{v, v}\right|_{H^{3 k-\frac{5}{2}}(\Omega)} & =\left|-N_{\mathcal{H}} \cdot \nabla \operatorname{tr}(D v)^{2}+2 D^{2} p_{v, v} \cdot D N_{\mathcal{H}}\right|_{H^{3 k-\frac{5}{2}}(\Omega)} \\
& \leq C\left(1+|\kappa|_{H^{3 k-2}(\partial \Omega)}\right)|v|_{H^{3 k}(\Omega)}^{2} .
\end{aligned}
$$

Therefore. we obtain the estimates on $\nabla_{N_{\mathcal{H}}} p_{v, v}$

The estimate of $D^{2} p_{v, v}$ is also achieved similarly. Firstly,

$$
\left|\Delta D^{2} p_{v, v}\right|_{H^{3 k-\frac{7}{2}(\Omega)}}=\left|D^{2} \operatorname{tr}(D v)^{2}\right|_{H^{3 k-\frac{7}{2}(\Omega)}} \leq C|v|_{H^{3 k}(\Omega)} .
$$

For the boundary value of $D^{2} p_{v, v}$, we first consider $D^{2} p_{v, v}(X, X)$ at $x \in \partial \Omega$ with $X \in T_{x} \partial \Omega$. As usual, extend $X$ to a vector fields in a neighborhood of $x$ on $\partial \Omega$ by parallel transporting $X$ along geodesics emitting from $x$. Thus,

$$
D^{2} p_{v, v}(X, X)=\nabla_{X} \nabla_{X} p_{v, v}-\nabla_{\nabla_{X} X} p_{v, v}=\nabla_{N} p_{v, v} \Pi(X, X) .
$$

Also, we have

$$
\begin{aligned}
& D^{2} p_{v, v}(N, N)=\Delta p_{v, v}-\kappa \nabla_{N} p_{v, v}=-\operatorname{tr}(D v)^{2}-\kappa \nabla_{N} p_{v, v} \\
& D^{2} p_{v, v}(N, X)=\nabla_{X} \nabla_{N} p_{v, v}-\nabla_{\nabla_{X} N} p_{v, v}=\nabla_{X} \nabla_{N} p_{v, v} .
\end{aligned}
$$

Therefore, from the above estimate on $\nabla_{N_{\mathcal{H}}} p_{v, v}$, we obtain the estimate on $D^{2} p_{v, v}$.

The following lemma is the most important observation of this paper, which states that $J$ is a solution of the linearized Euler's equation up to lower order terms. For the rest of this section, let $Q$ denote a generic positive polynomial in $|v|_{H^{3 k}\left(\Omega_{t}\right)},|\kappa|_{H^{3 k-2}\left(\partial \Omega_{t}\right)}$, and $\epsilon|\kappa|_{H^{3 k-1}\left(\partial \Omega_{t}\right)}$ with coefficients depending only on the set $\Lambda_{0}$.

Lemma 4.4. Suppose a solution of the Euler's equation is given by $\Omega_{t} \in \Lambda_{0}$ with $\partial \Omega_{t} \in H^{s_{0}}$, $s_{0}=3 k$ if $\epsilon=0$ or $s_{0}=3 k+1$ if $\epsilon>0$, and $v(t, \cdot) \in H^{3 k}\left(\Omega_{t}\right)$, then we have

$$
\left|\mathscr{D}_{t}^{2} J+\mathscr{R}_{0}(v) J+\epsilon^{2} \mathscr{A} J\right|_{H^{3 k-3}\left(\Omega_{t}\right)} \leq\left(1+\left|\mathscr{D}_{t} J\right|_{H^{3 k-3}\left(\Omega_{t}\right)}\right) Q .
$$

Proof. We start our proof by two simple observations. First we recall that for any vector field $w$ defined on $\Omega_{t}$ satisfying $\nabla \cdot w=0$, we write $p_{v, w}=-\Delta^{-1} \operatorname{tr}(D v D w)$

$$
\left|p_{v, w}\right|_{H^{s+1}\left(\Omega_{t}\right)} \leq C|v|_{H^{3 k}\left(\Omega_{t}\right)}|w|_{H^{s}\left(\Omega_{t}\right)}, \quad s \in[0,3 k-1],
$$


where $C>0$ is uniform in $\Omega \in \Lambda_{0}$. Second the first order derivative $\mathscr{D}_{t} J$ is lower order since by (3.24) we have

$$
\left|\mathscr{D}_{t} J-\mathbf{D}_{t} J\right|_{H^{s}\left(\Omega_{t}\right)} \leq Q \quad s \in[0,3 k-1],
$$

and by (3.18) and (3.6)

$$
\left|\mathbf{D}_{t} J\right|_{H^{3 k-3}\left(\partial \Omega_{t}\right)} \leq C|v|_{H^{3 k}\left(\Omega_{t}\right)} .
$$

To verify the lemma we consider the expression for $\mathscr{D}_{t}^{2} J$ given in (3.25) and keep the least regular terms. Thus

$$
\left|\mathscr{D}_{t}^{2} J-\mathbf{D}_{t}^{2} J\right|_{H^{3 k-3}\left(\Omega_{t}\right)} \leq Q+\left|\mathbf{D}_{t} \nabla p_{v, J}\right|_{H^{3 k-3}\left(\Omega_{t}\right)}
$$

$\mathbf{D}_{t} \nabla p_{v, J}$ is given by (3.26) and can be estimated using (4.12), lemma 4.3 and Euler's equation

$$
\left|\mathbf{D}_{t} \nabla p_{v, J}\right|_{H^{3 k-3}\left(\Omega_{t}\right)} \leq Q+C\left|D \mathbf{D}_{t} v \cdot D J\right|_{H^{3 k-4}\left(\Omega_{t}\right)} \leq Q .
$$

To estimate $\mathbf{D}_{t}^{2} J$ given in (3.20), we use (4.6) and Euler's equation to obtain

$$
\left|\mathbf{D}_{t}^{2} J-\nabla \mathbf{D}_{t}^{2} \kappa_{\mathcal{H}}\right|_{H^{3 k-3}\left(\Omega_{t}\right)} \leq Q .
$$

To estimate $\nabla \mathbf{D}_{t}^{2} \kappa_{\mathcal{H}}$ we use (3.21) and (3.22)

$$
\left|\nabla \mathbf{D}_{t}^{2} \kappa_{\mathcal{H}}-\nabla \mathcal{H}\left(\mathbf{D}_{t}^{2} \kappa\right)\right|_{H^{3 k-3}\left(\Omega_{t}\right)} \leq Q
$$

Combine these inequalities, we obtain

$$
\left|\mathscr{D}_{t}^{2} J-\nabla \mathcal{H}\left(\mathbf{D}_{t}^{2} \kappa\right)\right|_{H^{3 k-3}\left(\Omega_{t}\right)} \leq Q
$$

The term $\mathbf{D}_{t}^{2} \kappa$ has been calculated explicitly in (3.17) which yields,

$$
\left|\mathscr{D}_{t}^{2} J+\nabla \mathcal{H}\left(\Delta_{\partial \Omega_{t}} \mathbf{D}_{t} v \cdot N-2 \epsilon^{2} \Pi \cdot\left(\left.D^{\top}\right|_{T \partial \Omega_{t}} J\right)\right)\right|_{H^{3 k-3}\left(\Omega_{t}\right)} \leq Q .
$$

To deal with $\Delta_{\partial \Omega_{t}} \mathbf{D}_{t} v \cdot N$ we use Euler's equation to obtain that on the boundary

$$
\left.\Delta_{\partial \Omega_{t}} \mathbf{D}_{t} v \cdot N-2 \epsilon^{2} \Pi \cdot\left(\left.D^{\top}\right|_{T \partial \Omega_{t}} J\right)\right)=-N \cdot \Delta_{\partial \Omega_{t}}\left(\nabla p_{v, v}\right)-\epsilon^{2} \Delta_{\partial \Omega} \mathcal{N}(\kappa)+\epsilon^{2} J \cdot \Delta_{\partial \Omega_{t}} N .
$$

The last term can be bounded by the identity $\Delta_{\partial \Omega_{t}} N=-|\Pi|^{2} N+\nabla^{\top} \kappa$, and Lemma 4.2

$$
\left|\epsilon^{2} J \cdot \Delta_{\partial \Omega_{t}} N\right|_{H^{3 k-\frac{5}{2}}\left(\partial \Omega_{t}\right)}=\left.\left|\epsilon^{2}\right| \nabla^{\top} \kappa\right|^{2}-\left.\epsilon^{2} \mathcal{N}(\kappa)|\Pi|^{2}\right|_{H^{3 k-\frac{5}{2}}\left(\partial \Omega_{t}\right)}<Q
$$

Substituting the above into (4.15) and Lemma 4.2

$$
\left|\mathscr{D}_{t}^{2} J-\nabla \mathcal{H}\left(N \cdot \Delta_{\partial \Omega_{t}}\left(\nabla p_{v, v}\right)\right)+\epsilon^{2} \mathscr{A} J\right|_{H^{3 k-3}\left(\Omega_{t}\right)} \leq Q .
$$

As we are very close to the final desired form, the second term on the above left side has to be related to $\mathscr{R}_{0}$. Using formula (6.13), on $\partial \Omega_{t}$, we have

$$
\begin{aligned}
& -N \cdot \Delta_{\partial \Omega_{t}} \nabla p_{v, v}=-N \cdot \Delta \nabla p_{v, v}+\kappa N \cdot \nabla_{N} \nabla p_{v, v}+N \cdot D^{2}\left(\nabla p_{v, v}\right)(N, N) \\
= & N \cdot \nabla\left(\operatorname{tr}(D v)^{2}\right)+\nabla_{N}\left(\kappa \mathcal{H} \nabla_{N_{\mathcal{H}}} p_{v, v}+D^{2} p_{v, v}\left(N_{\mathcal{H}}, N_{\mathcal{H}}\right)\right)-\nabla_{N} p_{v, v} J^{\perp} \\
& -\kappa \nabla p_{v, v} \cdot \nabla_{N} N_{\mathcal{H}}-2 D^{2} p_{v, v}\left(N, \nabla_{N} N_{\mathcal{H}}\right)
\end{aligned}
$$

Keeping the least regular terms and using lemma 4.3, implies

$$
\begin{aligned}
\left|-N \cdot \Delta_{\partial \Omega_{t}} \nabla p_{v, v}+\nabla_{N} p_{v, v} J^{\perp}\right|_{H^{3 k-\frac{5}{2}}\left(\partial \Omega_{t}\right)} & \leq Q \\
& +\left|\nabla_{N}\left(\kappa_{\mathcal{H}} \nabla_{N_{\mathcal{H}}} p_{v, v}+D^{2} p_{v, v}\left(N_{\mathcal{H}}, N_{\mathcal{H}}\right)\right)\right|_{H^{3 k-\frac{5}{2}}\left(\partial \Omega_{t}\right)}
\end{aligned}
$$

Let $f=\kappa_{\mathcal{H}} \nabla_{N_{\mathcal{H}}} p_{v, v}+D^{2} p_{v, v}\left(N_{\mathcal{H}}, N_{\mathcal{H}}\right)$ defined on $\Omega_{t}$, since $\left.p_{v, v}\right|_{\partial \Omega_{t}}=0$ then

$$
\left.|f|_{\partial \Omega_{t}}\right|_{H^{3 k-\frac{3}{2}}\left(\partial \Omega_{t}\right)}=\left|\Delta p_{v, v}-\Delta_{\partial \Omega_{t}} p_{v, v}\right|_{H^{3 k-\frac{3}{2}\left(\partial \Omega_{t}\right)}}=\left|\operatorname{tr}(D v)^{2}\right|_{H^{3 k-\frac{3}{2}}\left(\partial \Omega_{t}\right)} \leq C|v|_{H^{3 k}\left(\Omega_{t}\right)}^{2} .
$$

Moreover it is easy to check from Lemma 4.3,

$$
|\Delta f|_{H^{3 k-3}\left(\Omega_{t}\right)} \leq Q
$$


which implies $\left|\nabla_{N} f\right|_{H^{3 k-\frac{5}{2}}\left(\partial \Omega_{t}\right)} \leq Q$. Therefore (4.17) together with the definition of $\mathscr{R}_{0}$ and the half derivative behavior of $\nabla \mathcal{H}$ implies

$$
\left.\left|\mathscr{R}_{0} J+\nabla \mathcal{H}\left(N \cdot \Delta_{\partial \Omega_{t}}\left(\nabla p_{v, v}\right)\right)\right|\right|_{H^{3 k-3}\left(\Omega_{t}\right)} \leq Q
$$

which together with (4.16) concludes the estimate in the statement of the lemma.

Proof of Theorems 4.1 and 4.2. To prove Theorem 4.1, in addition to Proposition 4.1, we need the following: a) the estimates on the Lagrangian coordinates map and consequently $\kappa \in H^{3 k-\frac{5}{2}}\left(\partial \Omega_{t}\right)$, b) estimates on $\omega=D v-(D v)^{*}$, and c) commutators involving $\mathbf{D}_{t}$, mostly have been done in Section 3. In the following all constant $C>0$ will be determined only by the set $\Lambda_{0}$.

Estimate of the Lagrangian coordinate map $u(t, y)$. From our assumption on $v$, the ODE $u_{t}(t, y)=$ $v(t, u(t, y))$ solving $u$ is well-posed. Since $u(t, \cdot): \Omega_{0} \rightarrow \Omega_{t}$ is volume preserving and $3 k>\frac{n}{2}+1$, it is easy to derive, for any $s \in[0,3 k]$, and $f \in H^{s}\left(\Omega_{t}\right)$

$$
|f \circ u(t, \cdot)|_{H^{s}\left(\Omega_{0}\right)} \leq C|f|_{H^{s}\left(\Omega_{t}\right)}|u(t, \cdot)|_{H^{3 k}\left(\Omega_{0}\right)}^{s},
$$

where $C>0$ depends only on $s$. The proof follows simply from induction and interpolation. By duality, for $s \in[0,3 k]$,

$$
|f \circ u(t, \cdot)|_{H^{-s}\left(\Omega_{0}\right)} \leq C|f|_{H^{-s}\left(\Omega_{t}\right)}\left|u(t, \cdot)^{-1}\right|_{H^{3 k}\left(\Omega_{0}\right)}^{s} .
$$

Therefore,

$$
|u(t, \cdot)-I|_{H^{3 k}\left(\Omega_{0}\right)} \leq C \int_{0}^{t}\left|v\left(t^{\prime}, \cdot\right)\right|_{H^{3 k}\left(\Omega_{t}\right)}\left|u\left(t^{\prime}, \cdot\right)\right|_{H^{3 k}\left(\Omega_{0}\right)}^{3 k} d t^{\prime},
$$

where $C>0$ depends only on $n$ and $k$. Let $\mu>0$ be a positive large number to be specified later,

$$
t_{0}=\sup \left\{\left.t|| v\left(t^{\prime}, \cdot\right)\right|_{H^{3 k}\left(\Omega_{t}\right)}<\mu, \forall t^{\prime} \in[0, t]\right\},
$$

We have $t_{0}>0$ due to the continuity of $v(t, \cdot)$ in $H^{3 k}\left(\Omega_{t}\right)$. Then, for all $t \in\left[0, t_{0}\right]$,

$$
|u(t, \cdot)-I|_{H^{3 k}\left(\Omega_{0}\right)} \leq \mu \int_{0}^{t}\left|u\left(t^{\prime}, \cdot\right)\right|_{H^{3 k}\left(\Omega_{0}\right)}^{3 k} d t^{\prime} .
$$

Therefore, from ODE estimates, there exists $t_{1}>0$ and $C_{2}>0$ which depend only on $|v(0, \cdot)|_{H^{3 k}\left(\Omega_{t}\right)}$ such that, for all $0 \leq t \leq \min \left\{t_{0}, t_{1}\right\}$,

$$
|u(t, \cdot)-I|_{H^{3 k}\left(\Omega_{0}\right)} \leq C_{2} t .
$$

It implies the mean curvature estimate, for all $0 \leq t \leq \min \left\{t_{0}, t_{1}\right\}$,

$$
|\kappa(t, \cdot)|_{H^{3 k-\frac{5}{2}}\left(\partial \Omega_{t}\right)} \leq|\kappa(0, \cdot)|_{H^{3 k-\frac{5}{2}}\left(\partial \Omega_{0}\right)}+C_{3} t .
$$

Here it is easy to see from local coordinates constructed in Section 6 that $C_{3}$ is determined only by $|v(0, \cdot)|_{H^{3 k}\left(\Omega_{t}\right)}$ and the set $\Lambda_{0}$. Therefore, there exists $t_{2}>0$ determined only by $|v(0, \cdot)|_{H^{3 k}\left(\Omega_{t}\right)}$ and the set $\Lambda_{0}$ such that $\Omega_{t} \in \Lambda_{0}$ for $0 \leq t \leq \min \left\{t_{0}, t_{2}\right\}$.

Evolution of the curl $\omega=D v-(D v)^{*}$. From equations (E) and (3.8), we have

$$
\mathbf{D}_{t} \omega=D \mathbf{D}_{t} v-\left(D \mathbf{D}_{t} v\right)^{*}+\left((D v)^{*}\right)^{2}-(D v)^{2}=\left((D v)^{*}\right)^{2}-(D v)^{2}=-(D v)^{*} \omega-\omega D v .
$$

It is clear how to obtain the estimate of $\omega$ in terms of $v$ : differentiating the above equation $3 k-1$ times, multiplying it by $D^{3 k-1} \omega$ and integrating it on $\Omega_{t}$, we have

$$
\frac{d}{d t} \int_{\Omega_{t}}|\omega|_{H^{3 k-1}\left(\Omega_{t}\right)}^{2} d x \leq C|v|_{H^{3 k}\left(\Omega_{t}\right)}|\omega|_{H^{3 k-1}\left(\Omega_{t}\right)}^{2} .
$$


The commutator involving $\mathbf{D}_{t}$. First, from (3.14) and (3.13), it is easy to verify that, for any function $f$ defined on $\partial \Omega_{t}$,

$$
\begin{array}{ll}
\left|\left[\mathbf{D}_{t}, \Delta_{\partial \Omega_{t}}\right]\right|_{L\left(H^{s_{1}}\left(\partial \Omega_{t}\right), H^{s_{1}-2}\left(\partial \Omega_{t}\right)\right)} \leq C|v|_{H^{3 k}\left(\Omega_{t}\right)} & s_{1} \in\left(\frac{7}{2}-3 k, 3 k-\frac{1}{2}\right], \\
\left|\left[\mathbf{D}_{t}, \mathcal{N}\right]\right|_{L\left(H^{s_{2}}\left(\partial \Omega_{t}\right), H^{s_{2}-1}\left(\partial \Omega_{t}\right)\right)} \leq C|v|_{H^{3 k}\left(\Omega_{t}\right)} & s_{2} \in\left(1,3 k-\frac{1}{2}\right] .
\end{array}
$$

To extend the range of $s_{2}$, we use the weak form of $\left[\mathbf{D}_{t}, \mathcal{N}\right]$ :

$$
\begin{aligned}
\int_{\partial \Omega_{t}} g & {\left[\mathbf{D}_{t}, \mathcal{N}\right] f d S=\int_{\partial \Omega_{t}} g\left((D v)-(D v)^{*}\right)\left(\nabla^{\top} f\right) \cdot N+g \mathcal{N}(f) \nabla_{N} v \cdot N d S } \\
& +\int_{\Omega_{t}} g_{\mathcal{H}} \nabla f_{\mathcal{H}} \cdot \Delta v-2 D v\left(\nabla f_{\mathcal{H}}\right) \cdot \nabla g_{\mathcal{H}}+\nabla g_{\mathcal{H}} \cdot \nabla \Delta_{D}^{-1}\left(2 D v \cdot D^{2} f_{\mathcal{H}}+\nabla f_{\mathcal{H}} \cdot \Delta v\right) d x .
\end{aligned}
$$

To conclude that the above estimate for $\left[\mathbf{D}_{t}, \mathcal{N}\right]$ holds for $s_{2}=\frac{1}{2}$. By interpolation,

$$
\left|\left[\mathbf{D}_{t}, \mathcal{N}\right]\right|_{L\left(H^{s}\left(\partial \Omega_{t}\right), H^{s-1}\left(\partial \Omega_{t}\right)\right)} \leq C|v|_{H^{3 k}\left(\Omega_{t}\right)}, \quad s \in\left[\frac{1}{2}, 3 k-\frac{1}{2}\right] .
$$

Evolution of E: first look. Recall the expression of $E_{R T}$ and $E$ written as $E=I_{1}+I_{2}$ :

$$
\begin{aligned}
& I_{1}=\frac{\epsilon^{2}}{2}\left|\mathscr{A}^{k-\frac{1}{2}} J\right|_{L^{2}\left(\Omega_{t}\right)}^{2}=\frac{\epsilon^{2}}{2} \int_{\partial \Omega_{t}} \kappa \cdot \mathcal{N}\left(-\Delta_{\partial \Omega_{t}} \mathcal{N}\right)^{2 k-1} \kappa d S \quad I_{2}=\frac{1}{2}\left|\mathscr{A}^{k-1} \mathscr{D}_{t} J\right|_{L^{2}\left(\Omega_{t}\right)}^{2}, \\
& E_{R T}=\frac{1}{2}<\left(\mathscr{R}_{0}(v)\right) \mathscr{A}^{k-1} J, \mathscr{A}^{k-1} J>_{L^{2}\left(\Omega_{t}\right)}=\frac{1}{2} \int_{\partial \Omega_{t}}-\nabla_{N} p_{v, v}\left|\left(-\mathcal{N} \Delta_{\partial \Omega_{t}}\right)^{k-1} \mathcal{N}(\kappa)\right|^{2} d S .
\end{aligned}
$$

Also recall that

Since

$$
\frac{d}{d t} d S=\left(\mathcal{D} \cdot v^{\top}+\kappa v^{\perp}\right) d S
$$

$$
\left.\nabla \cdot v\right|_{\partial \Omega_{t}}=\mathcal{D} \cdot v^{\top}+\kappa v^{\perp}+\nabla_{N} v \cdot N=0
$$

then

$$
\left|\kappa v^{\perp}+\mathcal{D} \cdot v^{\top}\right|_{H^{3 k-\frac{3}{2}}\left(\partial \Omega_{t}\right)}=\left|\nabla_{N} v \cdot N\right|_{H^{3 k-\frac{3}{2}}\left(\partial \Omega_{t}\right)} \leq C|v|_{H^{3 k}\left(\Omega_{t}\right)}
$$

and thus $\frac{d}{d t} d S$ would not complicate the estimates since $3 k \geq \frac{n}{2}+\frac{3}{2}$.

I: $\quad\left|\frac{d}{d t} I_{1}-\epsilon^{2}<\mathscr{A}^{k-\frac{1}{2}} J, \mathscr{A}^{k-\frac{1}{2}} \mathscr{D}_{t} J>_{L^{2}\left(\Omega_{t}\right)}\right| \leq Q$.

To prove the inequality I, we use (4.23) and (4.24) to obtain

$$
\left|\frac{d}{d t} I_{1}-\epsilon^{2}<\left(-\Delta_{\partial \Omega_{t}} \mathcal{N}\right)^{2 k-1} \kappa, \mathcal{N} \mathbf{D}_{t} \kappa>_{L^{2}\left(\partial \Omega_{t}\right)}\right| \leq Q,
$$

and from (4.12) and (4.13), we have

$$
\left|\mathscr{D}_{t} J-\nabla \mathcal{H}\left(\mathbf{D}_{t} \kappa\right)\right|_{H^{3 k-\frac{3}{2}}\left(\Omega_{t}\right)} \leq C|v|_{H^{3 k}\left(\Omega_{t}\right)}|J|_{H^{3 k-\frac{3}{2}}\left(\Omega_{t}\right)} .
$$

It implies the estimate $\mathbf{I}$ for $\frac{d}{d t} I_{1}$.

II: $\quad \frac{d}{d t} I_{2}-<\mathscr{A}^{k-1} \mathscr{D}_{t} J, \mathscr{A}^{k-1} \mathscr{D}_{t}^{2} J>_{L^{2}\left(\Omega_{t}\right)} \mid \leq Q$.

If $k=1$, which may happen when $n=2,3$,

$$
\frac{d}{d t} I_{2}=<\mathscr{D}_{t} J, \mathscr{D}_{t}^{2} J>_{L^{2}\left(\Omega_{t}\right)}
$$

where we used the fact $\left\langle\mathscr{D}_{t} J,\left(\mathscr{D}_{t}^{2}-\mathbf{D}_{t} \mathscr{D}_{t}\right) J>_{L^{2}\left(\Omega_{t}\right)}=0\right.$. If $k>1$,

$$
I_{2}=\frac{1}{2} \int_{\partial \Omega_{t}}\left(\mathscr{D}_{t} J\right)^{\perp} \cdot\left(-\Delta_{\partial \Omega_{t}}\right)\left(-\mathcal{N} \Delta_{\partial \Omega_{t}}\right)^{2 k-3}\left(\mathscr{D}_{t} J\right)^{\perp} d S .
$$


From (4.23) and (4.24), we obtain

$$
\left|\frac{d}{d t} I_{2}-\int_{\partial \Omega_{t}} \mathbf{D}_{t}\left(\left(\mathscr{D}_{t} J\right)^{\perp}\right) \cdot\left(-\Delta_{\partial \Omega_{t}}\right)\left(-\mathcal{N} \Delta_{\partial \Omega_{t}}\right)^{2 k-3}\left(\mathscr{D}_{t} J\right)^{\perp} d S\right| \leq Q
$$

On $\partial \Omega_{t}$,

$$
\mathbf{D}_{t}\left(\mathscr{D}_{t} J \cdot N\right)=\left(\mathbf{D}_{t} \mathscr{D}_{t} J\right) \cdot N-\nabla_{\left(\mathscr{D}_{t} J\right)^{\top}} v \cdot N=\left(\mathscr{D}_{t}^{2} J\right) \cdot N+\nabla_{N} p_{v, \mathscr{D}_{t} J}-\nabla_{\left(\mathscr{D}_{t} J\right)^{\top}} v \cdot N,
$$

which implies, along with (4.12), the estimate II for $I_{2}$.

III: $\quad\left|\frac{d}{d t} E_{R T}-<\mathscr{A}^{k-1} \mathscr{R}_{0}(v) J, \mathscr{A}^{k-1} \mathscr{D}_{t} J>_{L^{2}\left(\Omega_{t}\right)}\right| \leq Q$.

In general, for any function $f(t, \cdot)$ defined on $\partial \Omega_{t}$ with $\Omega_{t} \in \Lambda_{0}$ satisfying $\int_{\partial \Omega_{t}} f d S=0$, we have

$$
\frac{d}{d t} \int_{\partial \Omega_{t}}-\nabla_{N} p_{v, v} f^{2} d S=\int_{\partial \Omega_{t}}-\nabla_{N} p_{v, v} f^{2}\left(\kappa v^{\perp}+\mathcal{D} \cdot v^{\top}\right)-2 \nabla_{N} p_{v, v} f \mathbf{D}_{t} f-f^{2} \mathbf{D}_{t}\left(\nabla_{N} p_{v, v}\right) d S
$$

Therefore, we obtain from (4.6),

$$
\left|\frac{d}{d t} \int_{\partial \Omega_{t}}-\nabla_{N} p_{v, v} f^{2} d S-\int_{\partial \Omega_{t}}-2 \nabla_{N} p_{v, v} f \mathbf{D}_{t} f-f^{2} \mathbf{D}_{t}\left(\nabla_{N} p_{v, v}\right) d S\right| \leq Q .
$$

Commuting $\mathbf{D}_{t}$ with $\nabla$ and $\Delta^{-1}$ by (3.8) and (3.12),

$$
\begin{aligned}
& \mathbf{D}_{t}\left(N \cdot \nabla p_{v, v}\right)=N \cdot\left(\nabla \mathbf{D}_{t} p_{v, v}-(D v)^{*} \nabla p_{v, v}\right) \\
= & -\nabla_{N} p_{v, v} \nabla_{N} v \cdot N+\nabla_{N} \Delta^{-1}\left(2 D v \cdot D^{2} p_{v, v}+\Delta v \cdot \nabla p_{v, v}\right)-\nabla_{N} \Delta^{-1}\left(\mathbf{D}_{t} \operatorname{tr}(D v)^{2}\right),
\end{aligned}
$$

and using Euler's equation (2.11) to get

$$
\frac{1}{2} \mathbf{D}_{t} \operatorname{tr}(D v)^{2}=-\operatorname{tr}(D v)^{3}-D^{2} p_{v, v} \cdot D v-\epsilon^{2} D^{2} \kappa_{\mathcal{H}} \cdot D v .
$$

Therefore,

$$
\left|\frac{d}{d t} \int_{\partial \Omega_{t}}-\nabla_{N} p_{v, v} f^{2} d S-2 \int_{\partial \Omega_{t}}-\nabla_{N} p_{v, v} f \mathbf{D}_{t} f-\epsilon^{2} f^{2} \nabla_{N} \Delta^{-1}\left(D^{2} \kappa_{\mathcal{H}} \cdot D v\right) d S\right| \leq Q
$$

Substituting $f=\left(-\mathcal{N} \Delta_{\partial \Omega_{t}}\right)^{k-1} \mathcal{N}(\kappa)$, we obtain

$$
\left|\frac{d}{d t} E_{R T}-\int_{\partial \Omega_{t}}-\nabla_{N} p_{v, v}\left(-\mathcal{N} \Delta_{\partial \Omega_{t}}\right)^{k-1} \mathcal{N}(\kappa) \cdot \mathbf{D}_{t}\left(-\mathcal{N} \Delta_{\partial \Omega_{t}}\right)^{k-1} \mathcal{N}(\kappa) d S\right| \leq Q .
$$

From (4.23), (4.24), and (4.25),

$$
\left|\frac{d}{d t} E_{R T}-<\mathscr{R}_{0}(v) \mathscr{A}^{k-1} J, \mathscr{A}^{k-1} \mathscr{D}_{t} J>_{L^{2}\left(\Omega_{t}\right)}\right| \leq Q .
$$

In order to apply lemma 4.4 to the estimate III, we need to estimate

$$
\begin{aligned}
I_{3} \triangleq & <\mathscr{A}^{k-1} \mathscr{D}_{t} J, \mathscr{A}^{k-1} \mathscr{R}_{0}(v) J>_{L^{2}\left(\Omega_{t}\right)}-<\mathscr{R}_{0}(v) \mathscr{A}^{k-1} J, \mathscr{A}^{k-1} \mathscr{D}_{t} J>_{L^{2}\left(\Omega_{t}\right)} \\
= & \int_{\partial \Omega_{t}}\left(\mathscr{D}_{t} J\right)^{\perp} \cdot\left(-\Delta_{\partial \Omega_{t}} \mathcal{N}\right)^{2 k-2}\left(-\nabla_{N} p_{v, v} \mathcal{N} \kappa\right) \\
& -\left(\mathscr{D}_{t} J\right)^{\perp} \cdot\left(-\Delta_{\partial \Omega_{t}} \mathcal{N}\right)^{k-1}\left(\left(-\nabla_{N} p_{v, v}\right) \mathcal{N}\left(-\Delta_{\partial \Omega_{t}} \mathcal{N}\right)^{k-1} \kappa\right) d S .
\end{aligned}
$$

Our strategy will be to move $\mathcal{N}$ and the multiplication operator by $-\nabla_{N} p_{v, v}$ in the first integrand by commuting them with $\mathcal{N}$ and $\Delta_{\partial \Omega_{t}}$. Thus, we need to estimate $\left[\Delta_{\partial \Omega_{t}}, \nabla_{N} p_{v, v}\right],\left[\mathcal{N}, \nabla_{N} p_{v, v}\right]$, 
and $\left[\Delta_{\partial \Omega_{t}}, \mathcal{N}\right]$. Using Lemma $4.3,(6.12)$, and (6.16), we have,

$$
\begin{array}{ll}
\left|\left[\Delta_{\partial \Omega_{t}}, \nabla_{N} p_{v, v}\right]\right|_{L\left(H^{s}\left(\partial \Omega_{t}\right), H^{s-\frac{3}{2}}\left(\partial \Omega_{t}\right)\right)} \leq Q & s \in\left(3-3 k, \frac{n-1}{2}\right) \\
\left|\left[\mathcal{N}, \nabla_{N} p_{v, v}\right]\right|_{L\left(H^{s}\left(\partial \Omega_{t}\right), H^{s-\frac{1}{2}}\left(\partial \Omega_{t}\right)\right)} \leq Q & s \in\left[\frac{1}{2}, \frac{n-1}{2}\right) \\
\left|\left[\Delta_{\partial \Omega_{t}}, \mathcal{N}\right]\right|_{L\left(H^{s}\left(\partial \Omega_{t}\right), H^{s-\frac{5}{2}}\left(\partial \Omega_{t}\right)\right)} \leq C & s \in\left(\frac{7}{2}-3 k, 3 k-1\right) .
\end{array}
$$

Therefore we obtain $\left|I_{3}\right| \leq Q$, which implies the estimate III on $\frac{d}{d t} E_{R T}$.

Evolution of $\mathcal{E}$. Combining the estimates I, II, III, and Lemma 4.4, we obtain

$$
\left|\frac{d}{d t} \mathcal{E}\right| \leq Q, \quad \text { where } \quad Q=Q\left(|v|_{H^{3 k}\left(\Omega_{t}\right)},|\kappa|_{H^{3 k-2}\left(\partial \Omega_{t}\right)}, \epsilon|\kappa|_{H^{3 k-1}\left(\partial \Omega_{t}\right)}\right)
$$

is a polynomial with positive coefficients that depend only on $\Lambda_{0}$. This inequality on $\left[0, \min \left\{t_{0}, t_{2}\right\}\right]$ where $t_{0}$ is defined in (4.19) and $t_{2}$ is determined only by $|v(0, \cdot)|_{H^{3 k}\left(\Omega_{t}\right)}$ and the set $\Lambda_{0}$.

Proof of Theorem 4.2. Assume (RT) holds. From Proposition 4.1, (4.26) implies (4.8). In addition, by choosing $\mu$ large enough compared to the initial data, $t_{0}$ is bounded below by a constant $t^{*}>0$ depending only on $|v(0, \cdot)|_{H^{3 k}\left(\Omega_{0}\right)}$ and the set $\Lambda_{0}$. Theorem 4.2 follows immediately.

Proof of Theorem 4.1. Assume $\epsilon>0$. From inequality (4.26) and Proposition 4.1, we obtain

$$
E(t)-E(0)+E_{R T}(t)-E_{R T}(0) \leq \int_{0}^{t} Q_{\epsilon}\left(|v|_{H^{3 k}\left(\Omega_{t^{\prime}}\right)},|\kappa|_{H^{3 k-1}\left(\partial \Omega_{t^{\prime}}\right)}\right) d t^{\prime},
$$

where we use $Q_{\epsilon}$ to represent the dependence $Q$ on $\epsilon$. From proposition 6.6 and (4.6) we have

$$
\left|E_{R T}\right| \leq C\left|\nabla_{N} p_{v, v}\right|_{L^{\infty}\left(\partial \Omega_{t}\right)}|\kappa|_{H^{3 k-2}\left(\partial \Omega_{t}\right)}^{2} \leq C|v|_{H^{3 k-\frac{5}{8}\left(\Omega_{t}\right)}}^{2}|\kappa|_{H^{3 k-2}\left(\partial \Omega_{t}\right)}^{2} .
$$

Interpolating $v$ between $H^{3 k}\left(\Omega_{t}\right)$ and $H^{3 k-\frac{3}{2}}\left(\Omega_{t}\right)$ and $\kappa$ between $H^{3 k-1}\left(\partial \Omega_{t}\right)$ and $H^{3 k-\frac{5}{2}}\left(\partial \Omega_{t}\right)$, we obtain from Proposition 4.1,

$$
\left|E_{R T}\right| \leq \frac{1}{2} E+C_{1}\left(1+|v|_{H^{3 k-\frac{3}{2}\left(\Omega_{t}\right)}}^{m}\right)
$$

for some integer $m>0$ where the constant $C_{1}$, which include $|\kappa|_{H^{3 k-\frac{5}{2}}\left(\partial \Omega_{t}\right)}$, is determined only by $E_{0}$ and the set $\Lambda_{0}$. Since $\mathbf{D}_{t} v=-\nabla p_{v, v}-\epsilon^{2} J$ is controlled by $E$ in $H^{3 k-\frac{3}{2}}\left(\Omega_{t}\right)$ due to Proposition 4.1, we can use the Lagrangian coordinate map $u(t, \cdot)$ to estimate $|v(t, \cdot)|_{H^{3 k-\frac{3}{2}}\left(\Omega_{t}\right)}-|v(0, \cdot)|_{H^{3 k-\frac{3}{2}}\left(\Omega_{0}\right)}$. Through a similar procedure of the derivation of (4.20) and using Proposition 4.1, there exists $t_{3}>0$, depending only on $|v(0, \cdot)|_{H^{3 k}\left(\Omega_{t}\right)}$ and the set $\Lambda_{0}$ so that for $0 \leq t \leq \min \left\{t_{0}, t_{3}\right\}$,

$$
\left.|| v(t, \cdot)\right|_{H^{3 k-\frac{3}{2}\left(\Omega_{t}\right)}} ^{m}-|v(0, \cdot)|_{H^{3 k-\frac{3}{2}}\left(\Omega_{0}\right)}^{m} \mid \leq \int_{0}^{t} Q_{\epsilon} d t^{\prime}
$$

for some polynomial $Q_{\epsilon}$ with positive coefficients. Therefore,

$$
E_{R T} \leq \frac{1}{2} E+C_{1}\left(1+|v(0, \cdot)|_{H^{3 k-\frac{3}{2}}\left(\Omega_{0}\right)}^{m}\right)+\int_{0}^{t} Q_{\epsilon} d t^{\prime} \leq \frac{1}{2} E+C_{1}+\int_{0}^{t} Q_{\epsilon} d t^{\prime},
$$

where $C_{1}$ is determined only by $|v(0, \cdot)|_{H^{\frac{3}{2} k-\frac{3}{2}}\left(\Omega_{0}\right)}$ and the set $\Lambda_{0}$. Thus

$$
E\left(\Omega_{t}, v(t, \cdot)\right) \leq 2 E\left(\Omega_{0}, v(0, \cdot)\right)+C_{\epsilon}+\int_{0}^{t} Q_{\epsilon} d t^{\prime} .
$$


By inserting the above inequality into (4.27) and using proposition 4.1, we obtain (4.7). By choosing $\mu$ large enough compared to the initial data, Theorem 4.1 follows.

\section{Examples of Lagrangian CoORdinate maps Less SMOOTh Than $\partial \Omega_{t}$}

In Section 4, we established a priori estimates of the free boundary Euler's equation. In particular, the estimates indicate that $\partial \Omega_{t}$ is $\frac{1}{2}$, if $\epsilon=0$, or $\frac{3}{2}$, if $\epsilon>0$, derivative smoother than the velocity fields $\left.v\right|_{\partial \Omega_{t}}$. This is an improvement compared with the regularity directly given by the ODE defining the Lagrangian coordinate maps. It is very natural to guess that the Lagrangian coordinate maps might be smoother as well. However, the following examples show that the Lagrangian coordinate maps is only as smooth as the velocity fields.

5.1. Case 1: with surface tension. This is a relatively easy case for the construction of the example since we need not to worry about the sign assumption (RT): $-\nabla_{N} p_{v, v}>0$. The example is given for $n=2$ and $\Omega_{t}=B_{2}(1)$, the 2-dimensional open unit ball. In the polar coordinate, it is easy to verify that

$$
v(t, r, \theta)=\Theta(r) \frac{\partial}{\partial \theta}, \quad p(t, r, \theta)=\int_{r}^{1} r^{\prime} \Theta\left(r^{\prime}\right)^{2} d r^{\prime}, \quad \operatorname{supp}(\Theta) \subset \subset B_{2}(1) \backslash\{0\}
$$

is a stationary solution of $(\mathrm{E})$ and $(\mathrm{BC})$ for $\epsilon=1$. The Lagrangian coordinate map

$$
u\left(t, r_{0}, \theta_{0}\right)=\left(r_{0}, \theta_{0}+t \Theta\left(r_{0}\right)\right)
$$

is only as smooth as $v$.

5.2. Case 2: without surface tension. We will construct an example in $\mathbb{R}^{2}$ again, which satisfies the sign condition $-\nabla_{N} p>0$. Consider the domain and the vector field in the form

$$
\begin{aligned}
& \Omega_{t}=\left\{(r, \theta) \mid r_{1}(t)<r<r_{2}(t)\right\}, \quad r_{1}^{\prime}=\frac{a_{1}(t)}{r_{1}}, \quad r_{2}^{\prime}=\frac{a_{1}(t)}{r_{2}} \\
& v(t, r, \theta)=\frac{a_{1}(t)}{r} \frac{\partial}{\partial r}+\Theta(t, r) \frac{\partial}{\partial \theta},
\end{aligned}
$$

with the functions $a_{1}(t)$ and $\Theta(t, r)$ to be determined. In the polar coordinate

$$
\nabla_{\frac{\partial}{\partial r}} \frac{\partial}{\partial r}=0, \quad \nabla_{\frac{\partial}{\partial r}} \frac{\partial}{\partial \theta}=\nabla_{\frac{\partial}{\partial \theta}} \frac{\partial}{\partial r}=\frac{1}{r} \frac{\partial}{\partial \theta}, \quad \nabla_{\frac{\partial}{\partial \theta}} \frac{\partial}{\partial \theta}=-r \frac{\partial}{\partial r} .
$$

One may calculate

$$
D v\left(\frac{\partial}{\partial r}\right)=-\frac{a_{1}(t)}{r^{2}} \frac{\partial}{\partial r}+\left(\Theta_{r}+\frac{\Theta}{r}\right) \frac{\partial}{\partial \theta}, \quad D v\left(\frac{\partial}{\partial \theta}\right)=-r \Theta \frac{\partial}{\partial r}+\frac{a_{1}(t)}{r^{2}} \frac{\partial}{\partial \theta} .
$$

Thus, it is clear that the above given form ensures $v$ is divergence free:

$$
\nabla \cdot v=D v\left(\frac{\partial}{\partial r}\right) \cdot \frac{\partial}{\partial r}+D v\left(\frac{1}{r} \frac{\partial}{\partial \theta}\right) \cdot \frac{1}{r} \frac{\partial}{\partial \theta}=0 .
$$

Moreover, the above calculation implies

$$
\begin{aligned}
& \mathbf{D}_{t} v=v_{t}+\nabla_{v} v=\left(\frac{a_{1}^{\prime}(t)}{r}-\frac{a_{1}(t)^{2}}{r^{3}}-r \Theta^{2}\right) \frac{\partial}{\partial r}+\left(\Theta_{t}+\frac{a_{1}(t)}{r} \Theta_{r}+\frac{2 a_{1}(t)}{r^{2}} \Theta\right) \frac{\partial}{\partial \theta} \\
& -\Delta p=\operatorname{tr}\left((\nabla v)^{2}\right)=\frac{2 a_{1}(t)^{2}}{r^{4}}-2 r \Theta \Theta_{r}-2 \Theta^{2}=\frac{2 a_{1}(t)^{2}}{r^{4}}-\frac{1}{r}\left(r^{2} \Theta^{2}\right)_{r} .
\end{aligned}
$$

Therefore, $p$ must be radially symmetric, i.e. $p=p(t, r)$. It is straight forward to calculate

$$
-\nabla p=-p_{r} \frac{\partial}{\partial r}=-\left(\frac{a_{1}(t)^{2}}{r^{3}}+r \Theta^{2}+\frac{a_{2}(t)}{r}\right) \frac{\partial}{\partial r}
$$


for some function $a_{2}(t)$. From the boundary condition $p\left(t, r_{1}(t)\right)=p\left(t, r_{2}(t)\right)$, we need for the Euler's equation,

$$
\left\{\begin{array}{l}
a_{1}^{\prime}=-a_{2}=\left(\log \frac{r_{2}}{r_{1}}\right)^{-1}\left(\frac{a_{1}^{2}}{2}\left(\frac{1}{r_{1}^{2}}-\frac{1}{r_{2}^{2}}\right)+\int_{r_{1}}^{r_{2}} r \Theta^{2} d r\right) \\
\mathbf{D}_{t} \Theta+\frac{2 a_{1}(t)}{r^{2}} \Theta=\Theta_{t}+\frac{a_{1}(t)}{r} \Theta_{r}+\frac{2 a_{1}(t)}{r^{2}} \Theta=0 .
\end{array}\right.
$$

Let the Lagrangian coordinate map be $r=r\left(t, r_{0}, \theta_{0}\right)$ and $\theta=\theta\left(t, r_{0}, \theta_{0}\right)$, with $r_{0} \in\left[r_{1}(0), r_{2}(0)\right]$. Due to the symmetry of the vector field, we have $r=r\left(t, r_{0}\right)$ and $\theta=\theta_{0}+\theta_{1}\left(t, r_{0}\right)$ which satisfy

$$
\partial_{t} r=\frac{a_{1}(t)}{r} \quad \partial_{t} \theta_{1}=\Theta(t, r)
$$

It is easy to see

$$
r^{2}=r_{0}^{2}+2 A(t), \quad A(t)=\int_{0}^{t} a_{1}\left(t^{\prime}\right) d t^{\prime}
$$

Thus, the system (5.1) is equivalent to

$$
\left\{\begin{array}{l}
A^{\prime \prime}=\left(\log \frac{r_{2}(0)^{2}+2 A}{r_{1}(0)^{2}+2 A}\right)^{-\frac{1}{2}}\left(\frac{\left(A^{\prime}\right)^{2}}{2}\left(\frac{1}{r_{1}(0)^{2}+2 A}-\frac{1}{r_{2}(0)^{2}+2 A}\right)+\int_{r_{1}(0)}^{r_{2}(0)} r_{0}\left(\partial_{t} \theta_{1}\left(t, r_{0}\right)\right)^{2} d r_{0}\right) \\
\partial_{t t} \theta_{1}\left(t, r_{0}\right)=-\frac{2 A^{\prime}}{r_{0}^{2}+2 A} \partial_{t} \theta_{1}=-\left(\partial_{t} \log \left(r_{0}^{2}+2 A\right)\right) \partial_{t} \theta_{1} .
\end{array}\right.
$$

The system $(5.2)$ can be viewed as an ODE system for $\left(A, \theta_{1}(\cdot)\right)$ on an open set in the Banach space $X=\mathbb{R} \times C^{0}\left(\left[r_{1}(0), r_{2}(0)\right]\right)$. Therefore the unique existence of solution to this system is guaranteed. One can solve for

$$
\theta\left(t, r_{0}\right)=\theta_{0}+\Theta\left(0, r_{0}\right) \int_{0}^{t} \frac{r_{0}^{2}}{r_{0}^{2}+2 A(\tau)} d t^{\prime} \quad \Theta(t, r)=\frac{r^{2}-2 A}{r^{2}} \Theta\left(0, \sqrt{r^{2}-2 A}\right) .
$$

Thus, it is clear that the Lagrangian coordinate map is not smoother than the velocity field. Finally, one notices that small $\Theta$ would ensure the sign condition $p_{r}\left(t, r_{2}(t)\right)<0$ and $p_{r}\left(t, r_{1}(t)\right)>0$ since small $\Theta$ would make $v=\frac{a_{1}(t)}{r} \frac{\partial}{\partial r}+\Theta(t, r) \frac{\partial}{\partial \theta}$ a small perturbation of the irrotational solution $v=\frac{\tilde{a}_{1}(t)}{r} \frac{\partial}{\partial r}$, with a slightly different $\tilde{a}_{1}$, which satisfies the sign condition.

\section{Appendix I: Basic estimates}

In free boundary problems, it often happens that the moving domain $\Omega_{t}$ is of class $H^{s}$ and moves with an $H^{s_{0}}$ velocity field with $s_{0} \leq s$. Moreover, the estimates usually involve functions and vector fields defined on $\Omega_{t}$ and $\partial \Omega_{t}$. Therefore, in this section, we consider collections $\Lambda$ of domains $\Omega$ which are $H^{s_{0}}$ close to some reference domain and bounded in the $H^{s}$ class in some sense to be defined rigorously. We will outline some basic estimates on functions defined on $\Omega$ and $\partial \Omega$ and some related operators. Through tedious derivation, these estimates will be guaranteed to be uniform for all $\Omega \in \Lambda$.

6.1. Sobolev norms. Let $\Omega \subset \mathbb{R}^{n}$ be a bounded connected domain, viewing $H^{s}(\Omega), s \geq 0$, as a quotient space of $H^{s}\left(\mathbb{R}^{n}\right)$, define the norm

$$
|g|_{H^{s}(\Omega)}=\inf \left\{|G|_{H^{s}\left(\mathbb{R}^{n}\right)}: G \in H^{s}\left(\mathbb{R}^{n}\right),\left.G\right|_{\Omega}=g\right\}
$$

where $|\cdot|_{H^{s}\left(\mathbb{R}^{n}\right)}$ is defined through the Fourier transform. As usual, for $s \geq 0, H_{0}^{s}(\Omega)$ represents the closure of $C_{0}^{\infty}(\Omega)$ in $H^{s}(\Omega)$ and $H^{-s}(\Omega)$ is isometric to $\left(H_{0}^{s}(\Omega)\right)^{*}$. It is important to note that with this definition of $H^{s}$ norm the constants in Sobolev embedding $\left(H^{s} \rightarrow L^{p}\right.$ or $\left.C^{\alpha}\right)$ are independent of $\Omega$. The relationship between this definition of $H^{s}$ norm and the standard definition will be explored later on page 28 .

$C^{1} \cap H^{2}$ boundary $\partial \Omega$. To consider functions defined on $\partial \Omega$, let $\Omega \subset \mathbb{R}^{n}$ be a bounded connected domain with $\partial \Omega$ of class $H^{2} \cap C^{1}$. Consider the local graph coordinates of $\partial \Omega$ in orthonormal frames. When two coordinate charts of this type overlap, it is easy to verify that the transition map between these two local coordinate maps is also of $C^{1} \cap H^{2}$. Therefore, on $\partial \Omega$, the definitions of spaces 
$C^{1}(\partial \Omega) \cap H^{2}(\partial \Omega)$ of scalar functions and $C^{0}(\partial \Omega) \cap H^{1}(\partial \Omega)$ of $(k, l)$-type tensors, though defined in local coordinates, are independent of the choice of local coordinates. The Christofell symbols and the usual geometric quantities of the hypersurface $\partial \Omega$, such as the second fundamental form and mean curvature are well-defined in $L^{2}(\partial \Omega)$ and the sectional curvature is in $L^{1}(\partial \Omega)$, for it is like the square of the second fundamental form. As these will be referred to later, we give the explicit formula in local coordinates here. Let $\left\{e_{1}, \ldots, e_{n}\right\}$ be an orthonormal frame and $\left(x^{1}, \ldots, x^{n}\right)$ be the coordinates associated with this frame. Suppose $\Omega$ locally is given by $x^{n}>f\left(x^{1}, \ldots, x^{n-1}\right)$ with $f \in H^{2} \cap C^{1}$, then using $\left(x^{1}, \ldots, x^{n-1}\right)$ as the local coordinates, we have

The Christofell symbols $\Gamma_{i j}^{k}=\frac{\partial_{k} f \partial_{i j} f}{\sqrt{1+|\nabla f|^{2}}}$;

The second fundamental form $\Pi\left(\frac{\partial}{\partial x^{i}}\right) \cdot \frac{\partial}{\partial x^{j}}=-\frac{\partial_{i j} f}{\sqrt{1+|\nabla f|^{2}}}$

Mean curvature $\kappa=-\partial_{j}\left(\frac{\partial_{j} f}{\sqrt{1+|\nabla f|^{2}}}\right)=-\frac{\Delta f}{\left(1+|\nabla f|^{2}\right)^{\frac{1}{2}}}+\frac{\partial_{i} f \partial_{j} f \partial_{i j} f}{\left(1+|\nabla f|^{2}\right)^{\frac{3}{2}}}$

Sectional curvature $\mathcal{R}\left(\frac{\partial}{\partial x^{i}}, \frac{\partial}{\partial x^{j}}, \frac{\partial}{\partial x^{i}}, \frac{\partial}{\partial x^{j}}\right)=\frac{\partial_{i i} f \partial_{j j} f-\left(\partial_{i j} f\right)^{2}}{1+|\nabla f|^{2}}$

Beltrami-Lapalace $\Delta_{\partial \Omega} \phi=\operatorname{tr} \mathcal{D}^{2} \phi=\mathcal{D} \cdot \nabla^{\top} \phi=\frac{1}{\sqrt{1+|\nabla f|^{2}}} \partial_{i}\left(g^{i j} \sqrt{1+|\nabla f|^{2}} \partial_{j} \phi\right) ;$

where the matrix $\left(g^{i j}\right)=\left(\delta_{i j}+\partial_{i} f \partial_{j} f\right)^{-1}$. For a $C^{0} \cap H^{1}$ tensor $T$ of $(k, l)$-type, the covariant derivatives $\mathcal{D} T$ is a $(k, l+1)$-type tensor in $L^{2}$. For any $(k, l)$ tensor $T_{1}$ and $(k, l+1)$ tensor $T_{2}$ in $C^{0} \cap H^{1}$, one may verify, possibly through smooth approximations of $\partial \Omega$,

$$
\int_{\partial \Omega}\left(\mathcal{D} T_{1}\right) \cdot T_{2} d S=\int_{\partial \Omega} \operatorname{tr}\left(T_{1} \cdot \mathcal{D} T_{2}(\cdot, \cdot)\right) d S .
$$

where, on the above right side, $\mathcal{D} T_{2}(\cdot, \cdot)$ denotes the $(k, l)$-type tensor define by $\mathcal{D} T_{2}(X, Y)(\cdots)=$ $\left(\mathcal{D}_{X} T_{2}\right)(Y, \cdots)$.

From this identity, for any $L^{2}$ tensor $T$, one can define $\mathcal{D} T$, in the distribution sense, as in the dual space of $C^{0} \cap H^{1}$ tensors. It is straightforward to verify that, for any $(k, l)$-type tensor $T$ in $C^{0} \cap H^{1}$, we have

$$
\int_{\partial \Omega} T \cdot \Delta_{\partial \Omega} T d S=-\int_{\partial \Omega}|\mathcal{D} T|^{2} d S
$$

If $T, \mathcal{D} T \in C^{0} \cap H^{1}$, we have

$$
\begin{aligned}
\int_{\partial \Omega}\left|\Delta_{\partial \Omega} T\right|^{2} d S= & \int_{\partial \Omega}\left|\mathcal{D}^{2} T\right|^{2}+\left[\mathcal{D}_{\mathcal{R}\left(X_{j_{1}}, X_{j_{2}}\right) X_{j_{1}}} T-\mathcal{R}\left(X_{j_{1}}, X_{j_{2}}\right) \mathcal{D}_{X_{j_{1}}} T\right] \cdot \mathcal{D}_{X_{j_{2}}} T \\
& -\frac{1}{2}\left|\mathcal{R}\left(X_{j_{1}}, X_{j_{2}}\right) T\right|^{2} d S
\end{aligned}
$$

Here $\left\{X_{1}, \ldots, X_{n-1}\right\}$ is any pointwise orthonormal frame of $\partial \Omega$ which always appears in the trace form resulting in the independence of the corresponding quantities of the choice of the frame. The curvature acts on $T$ in the usual sense

$$
\mathcal{R}(X, Y) T=\mathcal{D}_{Y} \mathcal{D}_{X} T-\mathcal{D}_{X} \mathcal{D}_{Y} T-\mathcal{D}_{[Y, X]} T=\mathcal{D}^{2} T(Y, X)-\mathcal{D}^{2} T(X, Y)
$$

Though $\mathcal{R}(X, Y) T$ seems to contain derivatives of $T$, one may calculate

$$
(\mathcal{R}(X, Y) T)\left(X_{1}, X_{2}, \ldots\right)=-T\left(\mathcal{R}(X, Y) X_{1}, X_{2}, \ldots\right)-T\left(X_{1}, \mathcal{R}(X, Y) X_{2}, \ldots\right)-\ldots
$$

So the dependence of $\mathcal{R}(X, Y) T$ on $X, Y, T$ is only pointwise and $\mathcal{R}$ vanishes if $T$ is a scalar function. 
Since $I-\Delta_{\partial \Omega}$ is a positive self-adjoint operator on $L^{2}(\partial \Omega)$, for $\phi: \partial \Omega \rightarrow \mathbb{R}$ and $r \geq 0$, we define the Sobolev norm $|\cdot|_{H^{r}(\partial \Omega)}$ on the surface $\partial \Omega$ as

$$
|\phi|_{H^{r}(\partial \Omega)}^{2}=\int_{\partial \Omega}\left|\left(I-\Delta_{\partial \Omega}\right)^{\frac{r}{2}} \phi\right|^{2} d S ; \quad|\cdot|_{L^{2}(\partial \Omega)}=|\cdot|_{H^{0}(\partial \Omega)} .
$$

As usual, for $r \geq 0,|\cdot|_{H^{-r}(\partial \Omega)}$ coincides with $|\cdot|_{H^{r}(\partial \Omega)^{*}}$. One may note here, since the Christofell symbols $\Gamma_{i j}^{k}$ are only in $L^{2}$, while $|T|_{H^{1}(\partial \Omega)}<\infty$ implies that $\mathcal{D} T \in L^{2}(\partial \Omega)$ from (6.2), it does not imply that $T$ is in $H^{1}$ in local coordinates, except when $T$ is of $(0,0)$-type, i.e. a scalar function. Similarly, from (6.3), $|T|_{H^{2}(\partial \Omega)}<\infty$ does not imply $\mathcal{D}^{2} T \in L^{2}(\partial \Omega)$.

Remark. When $n=2, \partial \Omega$ is 1-dimension and $\Delta_{\partial \Omega}=\partial_{s s}$ where $s$ is the arc length parameter, which is well defined if $\partial \Omega$ is in $W^{1,1}$. In fact, $\partial \Omega \in H^{s}, s>\frac{3}{2}$, is sufficient for the definitions of all the objects intrinsic in $\partial \Omega$.

$H^{s}$ boundary $\partial \Omega, s>\frac{n+1}{2}$. For the purpose of this paper, we assume $\partial \Omega \subset \mathbb{R}^{n}$ is in $H^{s}$ with $s>\frac{n+1}{2}$. On the one hand, we defined the norm $|\cdot|_{H^{r}(\partial \Omega)}$ using the Beltrami-Lapalace $\Delta_{\partial \Omega}$ in the above. On the other hand, an obvious and traditional way to define the Sobolev space $H^{r}(\partial \Omega)$, $-s \leq r \leq s$ for scalar valued functions and $1-s \leq r \leq s-1$ for tensor valued functions, is through local coordinate coverings of $\partial \Omega$ and the definition of the Sobolev space $H^{r}\left(\mathbb{R}^{n-1}\right)$. From standard Sobolev inequalities, it is easy to see that the latter definition of the spaces $H^{r}(\partial \Omega)$ is actually independent of local coordinates and naturally induces a topology on $H^{r}(\partial \Omega)$. In particular, when $r \geq 0$ is an integer, straightforward calculation also shows that a function $f$ (or tensor field) belongs to $H^{r}(\partial \Omega)$ if and only if $f, \mathcal{D}^{r} f \in L^{2}(\partial \Omega)$. In fact, we have

Proposition 6.1. For $r \in[-s, s](r \in[1-s, s-1]$ for tensors $)$, the norm $|\cdot|_{H^{r}(\partial \Omega)}$ is equivalent to the norm on $H^{r}(\partial \Omega)$ defined by using local coordinates.

The proof of this proposition follows from the standard elliptic estimates using the local coordinates along with interpolation. In particular, when $s$ is an integer, one may also prove it geometrically. In fact, the proposition clearly holds for $r=1$ and $r=2$ due to (6.2) and (6.3) and Sobolev inequalities. When $r$ is an integer and $r \in[3, s](r \in[3, s-1]$ for tensors), the proposition can be proved by using the following identity

$$
\left(\left[\Delta_{\partial \Omega}, \mathcal{D}\right] T\right)(X)=\mathcal{R}\left(X, X_{j}\right) \mathcal{D}_{X_{j}} T+\left(\mathcal{D}_{X_{j}} \mathcal{R}\right)\left(X, X_{j}\right) T+\left(\mathcal{R}\left(X, X_{j}\right) \mathcal{D} T\right)\left(X_{j}\right) .
$$

Finally, for non-integer or negative $r$, the proposition follows from interpolation and duality. Another implication of (6.4) is that $\mathcal{D}: H^{r}(\partial \Omega) \rightarrow H^{r-1}(\partial \Omega)$ is bounded and $|\cdot|_{L\left(H^{r}(\partial \Omega), H^{r-1}(\partial \Omega)\right)}$ depends only on $\mathcal{R}$ and its derivatives.

It is well known that the regularity of $\partial \Omega$ can be determined from the regularity of its mean curvature $\kappa$.

Proposition 6.2. Let $\Omega \subset \mathbb{R}^{n}$ be a domain such that $\partial \Omega \in H^{s_{0}}, s_{0}>\frac{n+1}{2}$. Suppose $|\kappa|_{H^{s-2}(\partial \Omega)}<$ $\infty$ with $s>s_{0}$, then $\partial \Omega \in H^{s}$.

Proposition 6.2 can be proved by using local coordinates and standard quasilinear estimates. Another proof can be based on the following identity which is also used in a priori estimates. Intuitively, let $e: \partial \Omega \rightarrow \mathbb{R}^{n}$ be the imbedding, then $\Pi=-N \cdot \mathcal{D}^{2} e$ and $\kappa=-N \cdot \Delta_{\partial \Omega} e$, where $\Pi$ is viewed as a symmetric quadratic form. Thus it is expected that the difference $\Delta_{\partial \Omega} \Pi-\mathcal{D}^{2} \kappa$ should be of lower order terms only. In fact,

$$
-\Delta_{\partial \Omega} \Pi=-\mathcal{D}^{2} \kappa+|\Pi|^{2} \Pi-\kappa \Pi^{2} .
$$

To prove the identity, at any $x \in \partial \Omega$, let $\lambda_{i}, i=1, \ldots, n-1$, be the eigenvalues of $\Pi(x)$ and $X_{i}$ be the associated eigenvectors which form an orthonormal frame of $T_{x} \partial \Omega$. Parallel transport this frame to every base point in a neighborhood of $x$ on $\partial \Omega$ along the radial geodesics emitting from 
$x$. From the construction, we have $\mathcal{D}_{X_{i}} X_{j}=0,\left[X_{i}, X_{j}\right]=0$, and $\Pi\left(X_{i}\right)=\lambda_{i} X_{i}$ at $x$. For any $X=a^{j} X_{j}$ with constants $a^{1}, \ldots, a^{n-1}$, at $x$,

$$
\begin{aligned}
\left(\Delta_{\partial \Omega} \Pi\right)(X, X) & =\left(\mathcal{D}_{X_{i}} \mathcal{D}_{X_{i}} \Pi\right)(X, X)=\nabla_{X_{i}}\left(\left(\mathcal{D}_{X_{i}} \Pi\right)(X, X)\right)=\nabla_{X_{i}}\left(\left(\mathcal{D}_{X} \Pi\right)\left(X_{i}, X\right)\right) \\
& =\left(\mathcal{D}_{X_{i}} \mathcal{D}_{X} \Pi\right)\left(X_{i}, X\right)=\left(\mathcal{D}_{X} \mathcal{D}_{X_{i}} \Pi\right)\left(X, X_{i}\right)+\left(\mathcal{R}\left(X, X_{i}\right) \Pi\right)\left(X_{i}, X\right) \\
& =\nabla_{X}\left(\left(\mathcal{D}_{X_{i}} \Pi\right)\left(X, X_{i}\right)\right)+\Pi\left(\mathcal{R}\left(X_{i}, X\right) X_{i}, X\right)+\Pi\left(X_{i}, \mathcal{R}\left(X_{i}, X\right) X\right) .
\end{aligned}
$$

For the first term at $x$, from the construction of our special frame,

$$
\nabla_{X}\left(\left(\mathcal{D}_{X_{i}} \Pi\right)\left(X, X_{i}\right)\right)=\nabla_{X}\left(\left(\mathcal{D}_{X} \Pi\right)\left(X_{i}, X_{i}\right)\right)=\nabla_{X} \nabla_{X} \kappa-2 \Pi\left(X_{i}, \mathcal{D}_{X} \mathcal{D}_{X} X_{i}\right)=\mathcal{D}^{2} \kappa(X, X) .
$$

To calculate the remaining two terms, one may substitute $X=a^{j} X_{j}$ and use $\Pi\left(X_{j}\right)=\lambda_{j} X_{j}$, the symmetry of $\mathcal{R}$, and the following calculation

$$
\begin{aligned}
4 \mathcal{R}\left(X_{i}, X_{j_{1}}\right) X_{i} \cdot X_{j_{2}}= & \mathcal{R}\left(X_{i}, X_{j_{1}}+X_{j_{2}}\right) X_{i} \cdot\left(X_{j_{1}}+X_{j_{2}}\right)-\mathcal{R}\left(X_{i}, X_{j_{1}}-X_{j_{2}}\right) X_{i} \cdot\left(X_{j_{1}}-X_{j_{2}}\right) \\
= & \Pi\left(X_{i}, X_{i}\right) \Pi\left(X_{j_{1}}+X_{j_{2}}, X_{j_{1}}+X_{j_{2}}\right)-\Pi\left(X_{i}, X_{j_{1}}+X_{j_{2}}\right)^{2} \\
& -\Pi\left(X_{i}, X_{i}\right) \Pi\left(X_{j_{1}}-X_{j_{2}}, X_{j_{1}}-X_{j_{2}}\right)+\Pi\left(X_{i}, X_{j_{1}}-X_{j_{2}}\right)^{2} \\
= & 4 \delta_{j_{1} j_{2}} \lambda_{i} \lambda_{j_{1}}-4 \delta_{i j_{1}} \delta_{i j_{2}} \lambda_{i}^{2} .
\end{aligned}
$$

Equality (6.5) follows consequently.

$H^{s_{0}}$ neighborhoods of domains, $s_{0}>\frac{n+1}{2}$. Given a domain $\Omega_{*}$ with $\partial \Omega_{*}$ in $H^{s_{0}}$, we will consider the set $\Lambda \triangleq \Lambda\left(\Omega_{*}, s_{0}, s, L, \delta\right), s \geq s_{0}$, of neighboring domains of $\Omega_{*}$, given in Definition 4.2. From Proposition 6.2 , every $\Omega \in \Lambda$ is in $H^{s}$. Given $\Omega_{*}$ and sufficiently small $\delta>0$, in the following, we will derive some estimates with bounds $C$ uniform in $\Omega \in \Lambda$. Since $\partial \Omega_{*}$ is compact, for any $\sigma>0$, there exist $x_{i} \in \mathbb{R}^{n}$ and $d, d_{i} \in\left(0, \frac{1}{2}\right], i=1, \ldots, m$,

(B1) $B\left(\partial \Omega_{*}, d\right) \subset \cup_{i=1}^{m} R_{i}\left(d_{i}\right)$ where each $R_{i}(\cdot)=\tilde{R}_{i}(\cdot) \times I_{i}(\cdot) \subset \mathbb{R}^{n}$ with $\tilde{R}_{i}(\cdot)$ and $I_{i}(\cdot)$ being an open $(n-1)$-dimensional disk and an open perpendicular segment in $\mathbb{R}^{n}$, both centered at $x_{i}$ and of the given radius and half length, respectively;

(B2) For each $i, z=\left(z_{1}, \ldots, z_{n-1}, z_{n}\right)=\left(\tilde{z}, z_{n}\right)$ being an Euclidean coordinate system on $\tilde{R}_{i}(\cdot) \times$ $I_{i}(\cdot)$, there exists an $H^{s}$ function $f_{* i}: \tilde{R}_{i}\left(2 d_{i}\right) \rightarrow I_{i}$, so that

$$
\left|f_{* i}\right|_{C^{0}}<\sigma d_{i}, \quad\left|D f_{* i}\right|_{C^{0}}<\sigma, \quad \text { and } \quad \Omega_{*} \cap R_{i}\left(2 d_{i}\right)=\left\{z^{n}>f_{* i}(\tilde{z})\right\} .
$$

For any $\sigma>0$ with a fixed coordinate covering $\left\{R_{i}\left(d_{i}\right)\right\}_{i=1}^{m}$ of $\partial \Omega_{*}$ of the above type, it is clear that, when $\delta>0$ is sufficiently small, $\left\{R_{i}\left(d_{i}\right)\right\}_{i=1}^{m}$ is still a coordinate covering of any $\partial \Omega \in \Lambda$ satisfying (B1) and (B2) with coordinate functions $\left\{f_{i} \in H^{s}\right\}_{i=1}^{m}$. This will provide us some technical convenience in deriving estimates uniform in $\Omega \in \Lambda$. The following proposition is a refinement of Proposition 6.2.

Proposition 6.3. Given $\Omega_{*}$, there exists $\delta>0$ such that, for any $L>0$, there exists $C>0$ such that the second fundamental form of any $\Omega \in \Lambda$ satisfies

$$
|\Pi|_{H^{s-2}(\partial \Omega)}<C \text {. }
$$

Proof. The proof follows simply from the standard elliptic estimates and we will only give a sketch. With $\partial \Omega \in H^{s}$ due to Proposition 6.2 , we will use the above coordinate covering $\left\{R_{i}\left(d_{i}\right)\right\}_{i=1}^{m}$ and the coordinate functions $\left\{f_{i} \in H^{s}\left(\tilde{R}_{i}\left(2 d_{i}\right)\right)\right\}_{i=1}^{m}$, whose $H^{s_{0}}\left(\tilde{R}_{i}\left(2 d_{i}\right)\right)$ norms are uniformly bounded in $i$ and $\Omega$. Let $\gamma:[0,+\infty) \rightarrow[0,1]$ be a smooth cut-off function supported on $\left[0, \frac{3}{2}\right]$ and $\left.\gamma\right|_{\left[0, \frac{5}{4}\right]} \equiv 1$. On each $\tilde{R}_{i}\left(2 d_{i}\right)$, let

$$
\gamma_{i}(\tilde{z})=\gamma\left(\frac{|\tilde{z}|}{d_{i}}\right), \quad \kappa_{i}(\tilde{z})=\gamma_{i}(\tilde{z}) \kappa\left(\tilde{z}, f_{i}(\tilde{z})\right), \quad g_{i}=\gamma_{i} f_{i}
$$


where $\kappa$ is the mean curvature of $\partial \Omega$. It is clear from the definition of $\Lambda$ that $\left|\kappa_{i}\right|_{H^{s_{1}-2}\left(\tilde{R}_{i}\left(d_{i}\right)\right)}$ is bounded uniformly in $i$ and $\Omega$ for $s_{1}=\min \left\{s_{0}+2, s\right\}$. From the mean curvature formula (6.1),

$$
\begin{aligned}
-\Delta g_{i}+\frac{\partial_{j_{1}} f_{i} \partial_{j_{2}} f_{i}}{1+\left|\nabla f_{i}\right|^{2}} \partial_{j_{1} j_{2}} g_{i}= & \left(1+\left|\nabla f_{i}\right|^{2}\right)^{\frac{1}{2}} \kappa_{i}-\Delta \gamma_{i} f_{i}-2 D \gamma_{i} \cdot D f_{i} \\
& +\frac{\partial_{j_{1}} f_{i} \partial_{j_{2}} f_{i}}{1+\left|\nabla f_{i}\right|^{2}}\left(\partial_{j_{1} j_{2}} \gamma_{i} f_{i}+\partial_{j_{1}} \gamma_{i} \partial_{j_{2}} f_{i}+\partial_{j_{2}} \gamma_{i} \partial_{j_{1}} f_{i}\right)
\end{aligned}
$$

Since $\gamma$ is supported on $\left[0, \frac{3}{2}\right]$, without loss of generality, we may treat $f_{i}$ as compactly supported on the ball of radius $\frac{7 d_{i}}{4}$ because $f_{i}$ can always be replaced by $\tilde{\gamma}\left(\frac{|\tilde{z}|}{d_{i}}\right) f_{i}(\tilde{z})$ where $\tilde{\gamma}$ is a cut-off function supported on $\left[0, \frac{7}{4}\right]$ and $\left.\tilde{\gamma}\right|_{\left[0, \frac{3}{2}\right]}=1$. By partition of the unity and the Inverse Function Theorem, $\left(f_{1}, \ldots, f_{m}\right)$ can be expressed by $g=\left(g_{1}, \ldots, g_{m}\right)$ with the same regularity and similar estimates. Thus, dividing both sides of the above equation by $\left(1+\left|\nabla f_{i}\right|^{2}\right)^{\frac{1}{2}}$, it can be rewritten as

$$
-\Delta g+A_{j_{1} j_{2}}(x, g, \partial g) \partial_{j_{1} j_{2}} g=\kappa+G(x, g, \partial g)
$$

where $A_{j_{1} j_{2}}, G_{1}, G_{2}$ are smooth in their arguments and $A_{j_{1} j_{2}} \leq C \sigma^{2}$ so the left side is uniformly elliptic. In this form, the estimate on $|g|_{H^{s_{1}}\left(\mathbb{R}^{n-1}\right)}$ uniform in $\Omega$ is obtained following the standard theory of quasilinear elliptic equations. If $s_{1}<s$, this procedure can be carried out again with $s_{0}$ replaced by $s_{1}=s_{0}+2$. Thus the desired uniform estimates on $f_{i}$ in $H^{s}$ follow by repeating this procedure.

Remark. 1) One can also prove Proposition 6.3 based on (6.5).

2) A more careful estimate can be found in Lemma 4.2, when a more detailed relationship between $|\Pi|_{H^{s-2}(\partial \Omega)}$ and $|\kappa|_{H^{s-2}(\partial \Omega)}$ is given under certain conditions.

Using (6.2), (6.3), (6.4), and the above uniform estimate on $\Pi$, which implies the uniform estimate on the curvature $\mathcal{R}$, it is easy to prove that, for any tensor $T \in H^{r}(\partial \Omega), r \in[2-s, s-1](r \in[1-s, s]$ for scalars), we have

$$
|\mathcal{D} T|_{H^{r-1}(\partial \Omega)} \leq C|T|_{H^{r}(\partial \Omega)}
$$

for some $C$ uniform in $\Omega \in \Lambda$.

From the uniform estimates on those (uniformly fixed) local coordinates derived in the above proof, it is also clear that the constants in the Sobolev inequalities (e.g. $|\cdot|_{H^{s}(\partial \Omega)}$ to $L^{p}(\partial \Omega)$ or $C^{\alpha}(\partial \Omega)$ for $\left.s \leq k\right)$ are uniform in $\Omega \in \Lambda$. The two most used inequalities in this paper are for $f \in H^{s_{1}}(\partial \Omega)$ and $g \in H^{s_{2}}(\partial \Omega), s_{1} \leq s_{2}$,

$$
\begin{array}{ll}
|f g|_{H^{s_{1}+s_{2}-\frac{n-1}{2}}(\partial \Omega)} \leq C|f|_{H^{s_{1}}(\partial \Omega)}|g|_{H^{s_{2}(\partial \Omega)}}, & \text { if } s_{2}<\frac{n-1}{2} \text { and } 0<s_{1}+s_{2} \\
|f g|_{H^{s_{1}(\partial \Omega)}} \leq C|f|_{H^{s_{1}(\partial \Omega)}}|g|_{H^{s_{2}(\partial \Omega)}}, & \text { if } s_{2}>\frac{n-1}{2} \text { and } 0 \leq s_{1}+s_{2} .
\end{array}
$$

Similar inequalities hold for $f$ and $g$ defined in $\Omega$.

6.2. Dirichlet-Neumann operator. Given $\Omega_{*}$, in order to study the Dirichlet-Neumann operator for domains $\Omega \in \Lambda \triangleq \Lambda\left(\Omega_{*}, s_{0}, s, L, \delta\right)$, we need to first construct local coordinate maps on each $R_{i}\left(2 d_{i}\right)$ for each $\Omega$, which flatten $\partial \Omega$ and have estimates uniform in $\Omega \in \Lambda$, based on the above coordinates functions of $\partial \Omega$.

Local coordinates and partition of the unit. From Proposition $6.3, \partial \Omega \cap R_{i}\left(2 d_{i}\right)$ is represented as the graph of an $H^{s}$ function $f_{i}: \tilde{R}_{i}\left(2 d_{i}\right) \rightarrow \mathbb{R}$. Let $\phi=\gamma_{i} f_{i}$ with $\gamma_{i}$ defined in the previous proof. A standard way to extend $\phi$ to a function $\Phi \in H^{s+\frac{1}{2}}\left(\mathbb{R}^{n}\right)$ is through the Fourier transform 
with an appropriate constant $a$ :

$$
\hat{\Phi}\left(\xi^{1}, \ldots, \xi^{n}\right)=a \frac{\left(1+\left(\xi^{1}\right)^{2}+\ldots+\left(\xi^{n-1}\right)^{2}\right)^{s}}{\left(1+\left(\xi^{1}\right)^{2}+\ldots+\left(\xi^{n}\right)^{2}\right)^{s+\frac{1}{2}}} \hat{\phi}\left(\xi^{1}, \ldots, \xi^{n-1}\right)
$$

Since $s+\frac{1}{2}>\frac{n}{2}+1$ and $\Phi$ is bounded in $H^{s+\frac{1}{2}}$ uniformly in $i$ and $\Omega,|D \Phi|_{C^{0}}$ is also uniformly bounded. Therefore, there exists $b>0$ so that

$$
H_{i}\left(z^{1}, \ldots, z^{n}\right)=\left(z^{1}, \ldots, z^{n-1}, b z^{n}+\Phi\left(z^{1}, \ldots, z^{n}\right)\right)
$$

is a diffeomorphism so that $\left|H_{i}\right|_{H^{s+\frac{1}{2}}\left(\mathbb{R}^{n}\right)}$ and $\left|\left(H_{i}\right)^{-1}\right|_{H^{s+\frac{1}{2}}\left(\mathbb{R}^{n}\right)}$ are bounded uniformly in $i$ and $\Omega$. Let $G_{i}=\left(H_{i}\right)^{-1}$ and $g_{i}(z)$ be the $n$-the component of $G_{i}$, then $\left|g_{i}\right|_{H^{s+\frac{1}{2}\left(\mathbb{R}^{n}\right)}}, \partial_{z_{n}} g_{i}$, and $\left(\partial_{z_{n}} g_{i}\right)^{-1}$ are bounded uniformly in $i$ and $\Omega$. Obviously, there exists a uniform $\delta_{*}>0$ so that

$$
\left.\left(\tilde{R}_{i}\left(\frac{5}{4} d_{i}\right) \times I_{i}\left(\frac{5}{4} \delta_{*} d_{i}\right)\right) \cap \Omega=\tilde{R}_{i}\left(\frac{5}{4} d_{i}\right) \times I_{i}\left(\frac{5}{4} \delta_{*} d_{i}\right)\right) \cap\left\{g_{i}>0\right\} .
$$

Based on the local coordinate maps, we can construct partition of the unit satisfying estimates uniform in $\Omega$ if $\delta$ is small. In fact, take $\gamma, \xi: C^{\infty}([0,+\infty),[0,1])$ so that $\operatorname{supp}(\gamma) \subset\left[0, \frac{5}{4}\right],\left.\gamma\right|_{\left[0, \frac{9}{8}\right]} \equiv 1$, $\xi(r)=r$ for $r \geq \frac{2}{3}$, and $\left.\xi\right|_{\left[0, \frac{1}{3}\right]} \equiv \frac{1}{3}$. Define

$$
\tilde{\gamma}_{* i}(z)=\gamma\left(\frac{|\tilde{z}|}{d_{i}}\right) \gamma\left(\frac{\left|z_{n}\right|}{\delta_{*} d_{i}}\right), \quad \eta=\xi \circ \sum_{i=1}^{m}\left(\tilde{\gamma}_{* i} \circ G_{i}\right), \quad \gamma_{* i}=\frac{\tilde{\gamma}_{* i} \circ G_{i}}{\eta}, \quad \gamma_{* 0}=\left(1-\Sigma_{i=1}^{m} \gamma_{* i}\right) \chi(\Omega) .
$$

It is straight forward to verify that $\gamma_{* 0}, \gamma_{* 1}, \ldots, \gamma_{* m} \in H^{s+\frac{1}{2}}\left(\mathbb{R}^{n},[0,1]\right)$ satisfy

$$
\left.\left|\gamma_{* i}\right|_{H^{s+\frac{1}{2}}\left(\mathbb{R}^{n}\right)} \leq C, \quad \operatorname{supp}\left(\gamma_{* i}\right) \subset \tilde{R}_{i}\left(\frac{5}{4} d_{i}\right) \times I_{i}\left(\frac{5}{4} \delta_{*} d_{i}\right)\right),
$$

for $i=1, \ldots, m$, and $\left.\left(\sum_{i=0}^{m} \gamma_{* i}\right)\right|_{\Omega} \equiv 1$.

Remark. Using the above local coordinates and partition of unity we can establish the equivalence of the standard $H^{\ell}$ norm and the norm given in definition 6.1 for integer $\ell \in\left(-s-\frac{1}{2}, s+\frac{1}{2}\right)$. The ratio of the two norms is bounded above and below by two constants depending only on $\Lambda$.

Trace and Harmonic extension. Let $s_{1} \in\left(\frac{1}{2}, s+\frac{1}{2}\right]$. Using the partition of the unit and the above local coordinates, it is straight forward to obtain the trace operator estimate

$$
\left|\left(\left.\Psi\right|_{\partial \Omega}\right)\right|_{H^{s_{1}-\frac{1}{2}}(\partial \Omega)} \leq C|\Psi|_{H^{s_{1}}(\Omega)}
$$

for any $\Psi \in H^{s_{1}}(\Omega)$ where $C>0$ is uniform in $\Omega \in \Lambda$.

In order to obtain the estimate on the Harmonic extension operator, we first construct an extension for convenience. Let $s_{2} \in(0, s]$ and $\psi \in H^{s_{2}}(\partial \Omega)$. Take the same auxiliary functions $\gamma$ and $\xi$ used above. For each $1 \leq i \leq m$, let $\phi_{i}(\tilde{z})=\gamma\left(\frac{|\tilde{z}|}{d_{i}}\right) \psi\left(H_{i}(\tilde{z}, 0)\right)$ and $\Phi_{i}(z)$ be the extension of $\phi_{i}$, defined in the way of (6.8). Let

$$
\tilde{\Phi}_{i}(z)=\Phi_{i}(z) \gamma\left(\frac{|\tilde{z}|}{d_{i}}\right) \gamma\left(\frac{\left|z_{n}\right|}{\delta_{*} d_{i}}\right), \quad \Psi_{1}=\Sigma_{i=1}^{m} \tilde{\Phi}_{i} \circ G_{i}
$$

where $\Psi_{1}$ can be viewed as a function defined on $\mathbb{R}^{n} \supset \Omega$. Let

$$
\eta_{i}(z)=\gamma\left(\frac{|\tilde{z}|}{d_{i}}\right)^{2} \gamma\left(\frac{\left|z_{n}\right|}{\delta_{*} d_{i}}\right), \quad \eta=\xi \circ \Sigma_{i=1}^{m}\left(\eta_{i} \circ G_{i}\right), \quad \Psi=\frac{\Psi_{1}}{\eta} .
$$

It is easy to verify that $\Psi \in H^{s_{2}+\frac{1}{2}}\left(\mathbb{R}^{n}\right)$ is an extension of $\psi \in H^{s_{2}}(\partial \Omega)$ satisfying the estimate

$$
|\Psi|_{H^{s_{2}+\frac{1}{2}}\left(\mathcal{R}^{n}\right)} \leq C|\psi|_{H^{s_{2}}(\partial \Omega)}
$$

with $C$ uniform in $\Omega \in \Lambda$. 
Using the partition of the unit and the local coordinates we constructed above and following the standard procedure, we have

Lemma 6.4. There exists $C>0$ which depends only on the set $\Lambda$ so that, for $s_{1} \in\left[\frac{1}{2}, s\right]$

$$
\left|\Delta^{-1}\right|_{L\left(H^{s_{1}-\frac{3}{2}}(\Omega), H^{s_{1}+\frac{1}{2}}(\Omega)\right)}+|\mathcal{H}|_{L\left(H^{s_{1}}(\Omega), H^{s_{1}+\frac{1}{2}}(\Omega)\right)} \leq C .
$$

Dirichlet-Neumann operator. Following from the above estimate, the Dirichlet-Neumann operator $\mathcal{N}: H^{s_{1}}(\partial \Omega) \rightarrow H^{s_{1}-1}(\partial \Omega)$ can be defined and it has a uniform bound for $s_{1} \in(1, s]$. In fact, we can extend $\mathcal{N}$ into a weaker form defined on $H^{s_{1}}(\partial \Omega)$ for $s_{1} \geq \frac{1}{2}$. Given $f \in H^{\frac{1}{2}}(\partial \Omega)$, define $\mathcal{N}(f) \in H^{-\frac{1}{2}}(\partial \Omega)$ as

$$
<\psi, \mathcal{N}(f)>=\int_{\Omega} \nabla f_{\mathcal{H}} \cdot \nabla \psi_{\mathcal{H}} d x
$$

for any $\psi \in H^{\frac{1}{2}}(\partial \Omega)$. It is easy to prove that

1) $\mathcal{N}$ is self-adjoint in $L^{2}(\partial \Omega)$ with compact resolvent;

2) the $\operatorname{kernel} \operatorname{ker}(\mathcal{N})=\{$ const $\}$;

3) $C|f|_{H^{\frac{1}{2}(\partial \Omega)}} \geq|\mathcal{N}(f)|_{H^{-\frac{1}{2}}(\partial \Omega)} \geq \frac{1}{C}|f|_{H^{\frac{1}{2}(\partial \Omega)}}$ for any $f$ satisfying $\int_{\partial \Omega} f d S=0$.

The first inequality of 3 ) follows from the uniform bound on $\mathcal{H}$. In order to prove the second inequality in (3), one notices that

$$
|f|_{H^{\frac{1}{2}(\partial \Omega)}}|\mathcal{N}(f)|_{H^{-\frac{1}{2}(\partial \Omega)}} \geq|<f, \mathcal{N}(f)>|=\int_{\Omega}|\nabla \mathcal{H}(f)|^{2} d x .
$$

From the estimate of the trace operator, we only need, for any $f$ satisfying $\int_{\partial \Omega} f d S=0$,

$$
|\mathcal{H}(f)|_{L^{2}(\Omega)} \leq C|\nabla \mathcal{H}(f)|_{L^{2}(\Omega)}
$$

with a constant $C$ uniform in $f$ and $\Omega$. This inequality can be proved by a compactness argument. Thus, by duality and interpolation, $\mathcal{N}$ can be extended to $H^{s_{1}}(\partial \Omega)$ for all $s \in[1-s, s]$ and $|\mathcal{N}|_{L\left(H^{s_{1}}(\partial \Omega), H^{s_{1}-1}(\partial \Omega)\right)}$ is bounded uniformly in $\Omega$. Moreover, for $f \in H^{s}(\partial \Omega)$ with $\int_{\partial \Omega} f d S=0$, we can obtain $|f|_{H^{s}(\partial \Omega)} \leq C|\mathcal{N}(f)|_{H^{s-1}(\partial \Omega)}$ with $C$ uniform in $\Omega$. The proof is simply the elliptic estimate under the Neumann boundary condition - very much similar to the derivation of the harmonic extension estimate, except in the first step, instead of using (6.8), we need to construct $F$ with $|F|_{H^{s+\frac{1}{2}(\Omega)}} \leq C|\mathcal{N}(f)|_{H^{s-1}(\partial \Omega)}$ and $\nabla_{N} F=\mathcal{N}(f)$ on $\partial \Omega$, by using a slightly different formula of the same fashion. Therefore, from interpolation, we have, for any $s_{1} \in\left[\frac{1}{2}, s\right]$,

$$
|f|_{H^{s_{1}(\partial \Omega)}} \leq C|\mathcal{N}(f)|_{H^{s_{1}-1}(\partial \Omega)}, \quad \text { if } \int_{\partial \Omega} f d S=0
$$

with $C$ uniform in $\Omega$. moreover, this inequality holds for $s_{1} \in[1-s, s]$ by duality. Based on these estimates, we can use $I+\mathcal{N}$ to define the Sobolev norms which are equivalent to those defined by using $I-\Delta_{\partial \Omega}$ uniformly in $\Omega$, i.e.

Proposition 6.5. For $s_{1} \in[-s, s]$, the norms on $H^{s_{1}}(\partial \Omega)$ defined by interpolating $I-\Delta^{T}$ and $I+\mathcal{N}$ are equivalent, i.e.

$$
\frac{1}{C}\left(I-\Delta_{\partial \Omega}\right)^{\frac{s_{1}}{2}} \leq(I+\mathcal{N})^{s_{1}} \leq C\left(I-\Delta_{\partial \Omega}\right)^{\frac{s_{1}}{2}}
$$

with $C$ uniform in $\Omega \in \Lambda$.

Furthermore, for $s_{1} \in[-s, s-1]$,

$$
\mathcal{N}^{-1}: \dot{H}^{s_{1}}(\partial \Omega) \rightarrow \dot{H}^{s_{1}+1}(\partial \Omega), \quad \dot{H}^{s_{1}}(\partial \Omega)=\left\{f \in H^{s_{1}}(\partial \Omega) \mid \int_{\partial \Omega} f d S=0\right\}
$$


is well defined and bounded uniformly in $\Omega . \mathcal{N}^{-1}$ defined on $\cdot H^{-\frac{1}{2}}(\partial \Omega)$ induces the solvability of the Lapalace equation with Neumann boundary data given in $H^{-\frac{1}{2}}(\partial \Omega)$.

To demonstrate that $\mathcal{N}$ behaves like differentiation, we give the following "product rule". Given functions $f$ and $g$ defined on $\partial \Omega$. Since

$$
f_{\mathcal{H}} g_{\mathcal{H}}-\Delta^{-1} \Delta\left(f_{\mathcal{H}} g_{\mathcal{H}}\right)=\mathcal{H}\left(\left.f_{\mathcal{H}} g_{\mathcal{H}}\right|_{\partial \Omega}\right)=\mathcal{H}(f g) \quad \text { in } \Omega
$$

we obtain

$$
\mathcal{N}(f g)=f \mathcal{N}(g)+g \mathcal{N}(f)-2 \nabla_{N} \Delta^{-1}\left(\nabla f_{\mathcal{H}} \cdot \nabla g_{\mathcal{H}}\right)
$$

Since $\mathcal{N}$ is like differentiation, coordinate independent, and self-adjoint, appearing naturally in the Euler's equation, it is sometimes convenient to express the Sobolev norms on $\partial \Omega$ by $\mathcal{N}$.

Relationship between $\mathcal{N}$ and $\Delta_{\partial \Omega}$. In addition to just the comparison between the norms of $\Delta_{\partial \Omega}$ and $\mathcal{N}$, we will prove that $\mathcal{N}$ is simply equal to $\left(-\Delta_{\partial \Omega}\right)^{\frac{1}{2}}$ plus lower order terms. This improves the previous estimates and makes the estimates of some Sobolev norms using $\mathcal{N}$ more convenient. From the identity

$$
\Delta \psi=\Delta_{\partial \Omega} \psi+\kappa \nabla_{N} \psi+D^{2} \psi(N, N) \quad x \in \partial \Omega
$$

for any smooth function $\psi$ on $\Omega$. Recall that $N_{\mathcal{H}}(x)$ and $\kappa_{\mathcal{H}}(x), x \in \Omega$, denote the harmonic extension of the unit outward normal vector and the mean curvature of $\partial \Omega$. Given smooth $f$ : $\partial \Omega \rightarrow \mathbb{R}$, at any $x \in \partial \Omega$,

$$
\begin{aligned}
D^{2} f_{\mathcal{H}}(N, N) & =\nabla_{N} \nabla_{N_{\mathcal{H}}} f_{\mathcal{H}}-\nabla f_{\mathcal{H}} \cdot \nabla_{N} N_{\mathcal{H}} \\
& =\nabla_{N}\left(\mathcal{H}\left(\left.\left(\nabla_{N} f_{\mathcal{H}}\right)\right|_{\partial \Omega}\right)+(-\Delta)^{-1}(-\Delta)\left(\nabla_{N \mathcal{H}} f_{\mathcal{H}}\right)\right)-\mathcal{N}(N) \cdot\left(\mathcal{N}(f) N+\nabla^{\top} f\right) \\
& =\mathcal{N}^{2}(f)-2 \nabla_{N}(-\Delta)^{-1}\left(D N_{\mathcal{H}} \cdot D^{2} f_{\mathcal{H}}\right)-\mathcal{N}(N) \cdot\left(\mathcal{N}(f) N+\nabla^{\top} f\right)
\end{aligned}
$$

which implies

$$
\left(-\Delta_{\partial \Omega}-\mathcal{N}^{2}\right) f=\kappa \mathcal{N}(f)-2 \nabla_{N}(-\Delta)^{-1}\left(D N_{\mathcal{H}} \cdot D^{2} f_{\mathcal{H}}\right)-\mathcal{N}(N) \cdot\left(\mathcal{N}(f) N+\nabla^{\top} f\right) .
$$

Proposition 6.6. 1) For $s>\frac{n+3}{2}$, there exists $C>0$ uniform in $\Omega \in \Lambda$ such that we have

$$
\left|\Delta_{\partial \Omega}+\mathcal{N}^{2}\right|_{L\left(H^{s^{\prime}}(\partial \Omega), H^{s^{\prime}-1}(\partial \Omega)\right)} \leq C, \quad s^{\prime} \in[2-s, s-1] .
$$

2) For $s \in\left(\frac{n+1}{2}, \frac{n+3}{2}\right)$ and $s>2$, there exists $C>0$ uniform in $\Omega \in \Lambda$ such that we have

$$
\left|\Delta_{\partial \Omega}+\mathcal{N}^{2}\right|_{L\left(H^{s^{\prime}}(\partial \Omega), H^{s^{\prime}-\frac{n+5}{2}+s}(\partial \Omega)\right)} \leq C, \quad s^{\prime} \in\left(2-s, \frac{n+1}{2}\right) .
$$

Proof. For $s^{\prime}>\frac{n+5}{2}-s$, the above inequalities follow directly from (6.14) and the estimates on $\mathcal{H}$ and $\mathcal{N}$. Thus, by duality and interpolation, we only need to consider $s^{\prime}=\frac{1}{2}$ or $s^{\prime}=\frac{\alpha}{2}$ in each case, respectively. Let $f, g: \partial \Omega \rightarrow \mathbb{R}$ be smooth and harmonically extend into $\Omega$. Equality (6.14) yields $\int_{\partial \Omega} g\left(-\Delta_{\partial \Omega}-\mathcal{N}^{2}\right) f d S=\int_{\partial \Omega} \kappa g \mathcal{N}(f)+g \mathcal{N}(N) \cdot\left(\mathcal{N}(f) N+\nabla^{\top} f\right) d S-2 \int_{\Omega} D N_{\mathcal{H}}\left(\nabla g_{\mathcal{H}}\right) \cdot \nabla f_{\mathcal{H}} d x$ which is sufficient to establish the estimate.

Corollary. The proposition implies the commutator estimates

$$
\left|\left[\Delta_{\partial \Omega}, \mathcal{N}\right]\right|_{L\left(\left(H^{s^{\prime}}(\partial \Omega), H^{s^{\prime}-2}(\partial \Omega)\right)\right.} \leq C, \quad s^{\prime} \in[3-s, s-1]
$$

if $s>\frac{n+3}{2}$ and

$$
\left|\left[\Delta_{\partial \Omega}, \mathcal{N}\right]\right|_{L\left(\left(H^{s^{\prime}}(\partial \Omega), H^{s^{\prime}-\frac{n+7}{2}+s}(\partial \Omega)\right)\right.} \leq C, \quad s^{\prime} \in\left(3-s, \frac{n+1}{2}\right),
$$

if $s \in\left(\frac{n+1}{2}, \frac{n+3}{2}\right)$ and $s>2$. 
We need the following abstract result for a more careful estimate on $\mathcal{N}$.

Proposition 6.7. Let $X$ be a Hilbert space and $A$ and $B$ be (possibly unbounded) self-adjoint positive operators on $X$ so that $A^{-1} B$ and $A B^{-1}$ are bounded. Suppose $K=A^{2}-B^{2}$ satisfies that $K B^{-\alpha}$ is bounded with $\alpha \in[0,2)$, then $(A-B) B^{1-\alpha}$ is bounded.

Proof. Let $R=A-B$. Calculating $(B+R)^{2}=B^{2}+K$, we obtain

$$
-B R-R B=R^{2}-K,
$$

which implies

$$
\frac{d}{d t}\left(e^{-B t} R e^{-B t}\right)=e^{-B t}\left(R^{2}-K\right) e^{-B t} \geq-e^{-B t} K e^{-B t} \geq-C e^{-B t} B^{\alpha} e^{-B t} .
$$

Therefore,

$$
R \leq C_{1} \int_{0}^{\infty} e^{-B t} B^{\alpha} e^{-B t} d t=\frac{C_{1}}{2} B^{\alpha-1}
$$

Calculating $A^{2}=(A-R)^{2}+K$ with a similar procedure, we obtain

$$
R \geq-C_{2} \int_{0}^{\infty} e^{-A t} A^{\alpha} e^{-A t} d t=-\frac{C_{2}}{2} A^{\alpha-1} .
$$

Thus, the conclusion follows.

From Proposition 6.6 and Proposition 6.7, we obtain

Theorem 6.1. There exist $C>0$, which depends only on the set $\Lambda$ such that if $s>\frac{n+3}{2}$

$$
\left|\left(-\Delta_{\partial \Omega}\right)^{\frac{1}{2}}-\mathcal{N}\right|_{L\left(H^{s^{\prime}}(\partial \Omega)\right)} \leq C, \quad s^{\prime} \in[1-s, s-1]
$$

and if $s>2$ and $s \in\left(\frac{n+1}{2}, \frac{n+3}{2}\right)$, for $\alpha=\frac{n+5}{2}-s$,

$$
\left|\left(-\Delta_{\partial \Omega}\right)^{\frac{1}{2}}-\mathcal{N}\right|_{L\left(H^{s^{\prime}}(\partial \Omega), H^{s^{\prime}-\alpha+1}(\partial \Omega)\right)} \leq C, \quad s^{\prime} \in\left(1-s, \frac{n+1}{2}\right) .
$$

Proof. We will give the proof for the second case only as the proof for the first proof is similar. The estimate (6.17) follows directly from Proposition 6.6 and 6.7 for $s^{\prime} \in\left(1-s, \frac{n-1}{2}\right)$. To prove the estimate for $s^{\prime} \in\left[\frac{n-1}{2}, \frac{n+1}{2}\right)$, we observe that $s^{\prime}-2 \in\left(1-s, \frac{n-1}{2}\right)$ and we have

$$
\left|\left(I-\Delta_{\partial \Omega}\right)^{-1}\left(\left(-\Delta_{\partial \Omega}\right)^{\frac{1}{2}}-\mathcal{N}\right)\left(I-\Delta_{\partial \Omega}\right)\right|_{L\left(H^{\frac{n+1}{2}}(\partial \Omega), H^{\frac{n+1}{2}-\alpha+1}(\partial \Omega)\right)} \leq C .
$$

Thus (6.17) follows from the commutator estimate (6.16).

Decomposition of vector fields. We conclude this section by introducing the velocity field decomposition. Given an $L^{2}$ vector field $u: \Omega \rightarrow \mathbb{R}^{n}$, it is standard to decompose it into the divergence free part $v \in L^{2}$ and the gradient part $-\nabla p$ for $p \in H_{0}^{1}(\Omega)$. In fact,

$$
-\Delta p=\nabla \cdot u \quad v=u+\nabla p .
$$

For any divergence free vector field $v \in L^{2}(\Omega)$, the normal component on the boundary $v^{\perp} \triangleq v \cdot N$ : $\partial \Omega \rightarrow \mathbb{R}$ in $H^{-\frac{1}{2}}(\partial \Omega)$ is defined as

$$
<v^{\perp}, \psi>=\int_{\Omega} v \cdot \nabla \psi_{\mathcal{H}} d x
$$

for any $\psi \in H^{\frac{1}{2}}(\partial \Omega)$. By interpolation, for any $s_{1} \in\left[0, s-\frac{1}{2}\right]$,

$$
\left|v^{\perp}\right|_{H^{s_{1}-\frac{1}{2}}(\partial \Omega)} \leq C|v|_{H^{s_{1}}(\Omega)},
$$


with $C$ uniform in $\Omega \in \Lambda$. This induces a decomposition of $v$ into two divergence free parts, the rotation part $v_{r}$ and the irrotational (or gradient) part $v_{i r}$, as follows

$$
v_{i r}=\nabla \mathcal{H N}^{-1} v^{\perp}, \quad v_{r}=v-v_{i r} .
$$

It is easy to verify $v_{r}, v_{i r} \in L^{2}(\Omega)$ and

$$
\nabla \cdot v_{r}=\nabla \cdot v_{i r}=0, \quad<v_{r}, v_{i r}>=0, \quad v_{r}^{\perp}=0 .
$$

If $v$ is a divergence free velocity field, $v_{r}$ component is responsible of the internal rotation and $v_{i r}$ of the motion of the domain.

\section{Notation}

$\operatorname{tr}(A)$ : the trace of an operator.

$A^{*}$ : the adjoint operator of an operator.

$A_{1} \cdot A_{2}=\operatorname{tr}\left(A_{1}\left(A_{2}\right)^{*}\right)$, for two operators.

$B(S, \epsilon)=\cup_{x \in S} B(x, \epsilon)$ : an $\epsilon$-neighborhood of a set $S$.

$D$ and $\partial$ : differentiation with respect to spatial variables.

$\nabla f$ : the gradient vector of a scalar function $f$.

$\nabla_{X}$ : the directional directive in the direction $X$.

$\perp$ and $T$ : the normal and the tangential components of the relevant quantities.

$\mathbf{D}_{t}=\partial_{t}+v^{i} \partial_{x^{i}}$ the material derivative along the particle path.

$\mathbf{D}_{t}^{\top}$ : the projection of $\mathbf{D}_{t}$ to the tangent space of $\partial \Omega_{t} \subset \mathbb{R}^{n}$.

$N(t, x)$ : the outward unit normal vector of $\partial \Omega_{t}$ at $x \in \partial \Omega_{t}$.

$\Pi$ : the second fundamental form of $\partial \Omega_{t}, \Pi(t, x)(w)=\nabla_{w} N \in T_{x} \partial \Omega_{t}$.

$\Pi(X, Y)=\Pi(X) \cdot Y$.

$\kappa$ : the mean curvature of $\partial \Omega_{t}$, i.e. $\kappa=\operatorname{tr} \Pi$.

$f_{\mathcal{H}}=\mathcal{H}(f)$ : the harmonic extension of $f$ on $\Omega_{t}$.

$\mathcal{N}(f)=\nabla_{N} \mathcal{H}(f): \partial \Omega \rightarrow \mathbb{R}:$ the Dirichlet-Neumann operator.

$\bar{X}=X \circ u^{-1}$ the Lagrangian coordinates description of $X$.

$\mathcal{D}$ : the covariant differentiation on $\partial \Omega_{t} \subset \mathbb{R}^{n}$.

$\mathcal{D}_{w}=\nabla_{w}^{\top}$, for any $x \in \partial \Omega_{t} \quad w \in T_{x} \partial \Omega_{t}$.

$\mathcal{R}(X, Y), X, Y \in T_{x} \partial \Omega_{t}$ : the curvature tensor of $\partial \Omega_{t}$.

$\Delta_{\mathcal{M}} \triangleq \operatorname{tr} \mathcal{D}^{2}$ : the Beltrami-Lapalace operator on a Riemannian manifold $\mathcal{M}$.

$\Delta^{-1}$ : the inverse Laplacian with zero Dirichlet data.

$\Gamma=\left\{\phi: \Omega_{t} \rightarrow \mathbb{R}^{n} ;\right.$ volume preserving homeomorphism $\}$

$\overline{\mathscr{D}}$ : the covariant derivative on $\Gamma$,

$\mathscr{D}$ : represent $\overline{\mathscr{D}}$ in Eulerian coordinates.

$\overline{\mathscr{R}}$ : the curvature operator on $\Gamma$.

$\mathscr{R}$ : represent $\overline{\mathscr{R}}$ in Eulerian coordinates.

II: the second fundamental form of $\Gamma \subset L^{2}$

$\mathrm{II}_{u}\left(w_{1}, w_{2}\right)=\nabla \frac{\perp}{w_{1}} w_{2}$, for any $u \in \Gamma, \quad w_{1}, w_{2} \in T_{u} \Gamma$

$p_{v, w}=-\Delta^{-1} \operatorname{tr}(D v D w)$.

\section{REFERENCES}

[AM03] Ambrose, D. M., Well-posedness of vortex sheets with surface tension. SIAM J. Math. Anal. 35 (2003), no. $1,211-244$ (electronic)

[AM05a] Ambrose, D. M.; Masmoudi, N., The zero surface tension limit of two-dimensional water waves. Comm. Pure Appl. Math. 58 (2005), no. 10, 1287-1315.

[AM06] Ambrose, D. M.; Masmoudi, N., Well-Posedness of 3D Vortex Sheets With Surface Tension. Preprint.

[BHL93] Beale, J.T.; Hou, T.; Lowengrub, J.Growth rates for the linearized motion of fluid inter-faces away from equilibrium, Comm. Pure Appl. Math., 46, (1993) 1269-1301. 
[BG98] Beyer, K.; Günther, M., On the Cauchy problem for a capillary drop. I. Irrotational motion. Math. Methods Appl. Sci. 21 (1998), no. 12, 1149-1183.

[BG00] Beyer, K.; Günther, M., The Jacobi equation for irrotational free boundary flows. Analysis (Munich), 20, (2000), no. 3, 237-254.

[CL00] Christodoulou, D.; Lindblad, H., On the motion of the free surface of a liquid. Comm. Pure Appl. Math. 53 (2000), no. 12, 1536-1602.

[CS05] Coutand D.; Shkoller S., Well posedness of the free-surface incompressible Euler equations with or without surface tension. Preprint.

[CR85] Craig, W., An existence theory for water waves and the Boussinesq and Korteweg-de Vries scaling limits. Comm. Partial Differential Equations, 10 (1985), no. 8, 787-1003.

[EB87] Ebin, D., The equations of motion of a perfect fluid with free boundary are not well posed. Comm. Part. Diff. Eq., 10, (1987), 1175-1201

[IG01] Iguchi, T., Well-posedness of the initial value problem for capillary-gravity waves. Funkcial. Ekvac. 44 (2001), no. 2, 219-241

[LA05] Lannes, D., Well-posedness of the water-waves equations. J. Amer. Math. Soc., 18, (2005) 605654.

[LI05] Lindblad, H. Well-posedness for the motion of an incompressible liquid with free surface boundary. Ann. of Math., 162, (2005), no. 1, 109-194.

[NA74] Nalimov, V. I., The Cauchy-Poisson problem. (Russian) Dinamika Splosn. Sredy Vyp. 18 Dinamika Zidkost. so Svobod. Granicami (1974), 10-210, 254

[OT02] Ogawa, M., Tani, A., Free boundary problem for an incompressible ideal fluid with surface tension. Math. Models Methods Appl. Sci. 12, (2002), no. 12, 1725-1740.

[SC05] Schweizer, B., On the three-dimensional Euler equations with a free boundary subject to surface tension. Ann. Inst. H. Poincar Anal. Non Linaire 22 (2005), no. 6, 753-781.

[SW02] Schneider, G., Wayne, C. E., The rigorous approximation of long-wavelength capillary-gravity waves. Arch. Ration. Mech. Anal., 162 (2002), no. 3, 247-285.

[WU97] Wu, S., Well-posedness in Sobolev spaces of the full water wave problem in 2-D. Invent. Math. 130 (1997), no. $1,39-72$

[WU99] Wu, S., Well-posedness in Sobolev spaces of the full water wave problem in 3-D. J. Amer. Math. Soc. 12 (1999), no. 2, 445-495.

[YO82] Yosihara, H., Gravity waves on the free surface of an incompressible perfect fluid of finite depth. Publ. Res. Inst. Math. Sci. 18 (1982), no. 1, 49-96.

[YO83] Yosihara, H., Capillary-gravity waves for an incompressible ideal fluid. J. Math. Kyoto Univ. 23 (1983), no. 4, 649-694.

[ZZ06] Zhang, P., Zhang, Z. On the free boundary problem of 3-D incompressible Euler equations. Preprint.

${ }^{\dagger}$ Courant Institute of Mathematical Sciences, 251 Mercer Street, New York, NY 10012

E-mail address: shatah@cims.nyu.edu

*School of Mathematics, Georgia Institute of Technology, Atlanta, GA 30332

E-mail address: zengch@math.gatech.edu 